\title{
Pedicularis L. Genus: Systematics, Botany, Phytochemistry, Chemotaxonomy, Ethnopharmacology, and Other
}

\author{
Claudio Frezza ${ }^{1, *(0)}$, Alessandro Venditti ${ }^{2}{ }^{-}$, Chiara Toniolo $^{1}$, Daniela De Vita ${ }^{1}$, Ilaria Serafini ${ }^{2}$, \\ Alessandro Ciccòla ${ }^{2}$, Marco Franceschin ${ }^{2}$, Antonio Ventrone ${ }^{1}$, Lamberto Tomassini ${ }^{1}{ }^{1}$, \\ Sebastiano Foddai ${ }^{1}$, Marcella Guiso ${ }^{2}$, Marcello Nicoletti ${ }^{1}$, Armandodoriano Bianco ${ }^{2}$ (D) \\ and Mauro Serafini ${ }^{1}$ \\ 1 Dipartimento di Biologia Ambientale, Università di Roma “La Sapienza", Piazzale Aldo Moro 5, \\ 00185 Rome, Italy \\ 2 Dipartimento di Chimica, Università di Roma "La Sapienza", Piazzale Aldo Moro 5, 00185 Rome, Italy \\ * Correspondence: claudio.frezza@uniroma1.it; Tel.: +39-0649912194
}

Received: 28 June 2019; Accepted: 12 August 2019; Published: 27 August 2019

\begin{abstract}
In this review, the relevance of the plant species belonging to the Pedicularis L. genus has been considered from different points of view. Particular emphasis was given to phytochemistry and ethnopharmacology, since several classes of natural compounds have been reported within this genus and many of its species are well known to be employed in the traditional medicines of many Asian countries. Some important conclusions on the chemotaxonomic and chemosystematic aspects of the genus have also been provided for the first time. Actually, this work represents the first total comprehensive review on this genus.
\end{abstract}

Keywords: Pedicularis L. genus; Orobanchaceae family; phytochemistry; chemotaxonomy; ethnopharmacology

\section{Systematics}

Pedicularis L. is a genus of hemiparasitic plants, originally included in the Scrophulariaceae family but now belonging to the Orobanchaceae family [1]. The rest of the systematic classification is the following: order Scrophulariales, subclass Asteridae, class Magnoliopsida, division Magnoliophyta, superdivision Spermatophyta, subkingdom Tracheobionta. The genus comprises 568 accepted species, 335 synonymous species, and 450 unresolved species [2].

\section{Etymology of the Name}

The etymology of the genus name is Latin, with the term "pediculus" meaning "louse", which refers to the fact that, according to an ancient English belief, cattle which grazed on these plants were soon found to be infested with lice [3].

\section{Botany}

Plants of the genus Pedicularis are generally herbaceous and perennial, with a height which can reach up to $50 \mathrm{~cm}$. Annual or biennial species are quite rare but present. From the morphological standpoint, these species are characterized by big and fleshy roots, often taproots, which contain specific organs (haustoria) for their feeding on the lymph of the near plants. The stem is erect and ascendant and may be simple or branched (Figure 1). The leaves are basal and cauline. The former ones are disposed to form a rosette and are petiolate. The latter ones are opposite, alternated or verticillated, 
and sessile, instead. Both of these have a lanceolate shape and dentate margins which are rarely entire. Bracts are also present and are similar to the cauline leaves, even if they are smaller (Figure 1). More or less dense terminal spikes generally constitute the inflorescence. The flowers are big, hermaphrodite, zigomorphic, and tetrameric or pentameric. They can be sessile or pedunculated. The floreal formula is $X, K(5),[C(2+3), A 2+2], G(2)$, (superior), capsule. The calyx is gamosepalous, formed by five lobes that may be dentate or not. The corolla is gamopetalous and bilabiate with a cylindrical shape slightly compressed on its sides. Its color ranges from pink to white, passing through red, purple, and yellow. The androecium possesses four didinamous stamens with the filaments well included into the base of the corolla. The anthers are hidden among dense hairs and may be mucronate. The pollen maturation is contemporaneous to the stigma. The ovary is superior, formed by two carpels, and bilocular. The stylus is inserted in the ovary apex and is filiform. The stigma is simple and protruded beyond the corolla hat in order to avoid self-pollination (Figure 1). The fruit is an acuminated bivalve capsule with an oval-lanceolate shape (Figure 1). The seeds are numerous or not and present an angular geometry. Reproduction occurs through pollination by insects or dispersion [4,5].

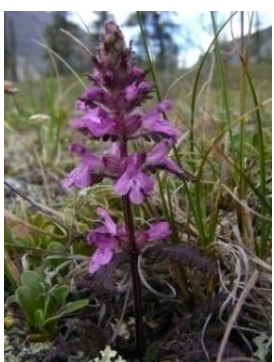

(a)

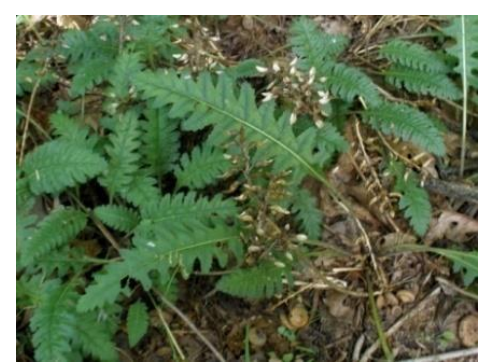

(b)

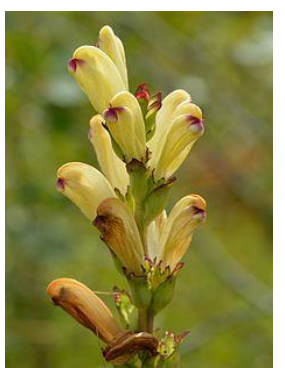

(c)

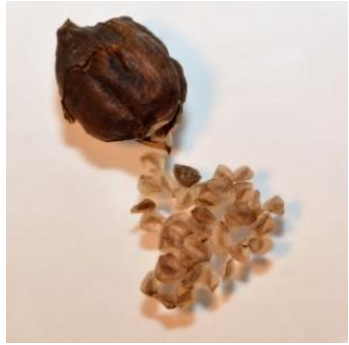

(d)

Figure 1. Examples of the morphological features of Pedicularis species—stem (a), leaves (b), flowers (c), fruits (d) [source Google images].

\section{Distribution and Habitat}

The species of this genus are distributed in Europe, especially in the mountainous areas of the Mediterranean Basin, and in Northern Asia and America (Figure 2). The highest biodiversity is present in Europe, with about 70 species, India, with about 83 species, and China, with about 350 species, 271 of which are endemic [6-8]. In North America, the present species are 36 with two endemisms [9]. These species have been reported in Africa and Australia only as imported plants. The preferred habitat is a temperate mountainous one. The soil must be quite acidic and little- draining. The typical areas where these species can be found are meadows and lawns with little other vegetation [3].

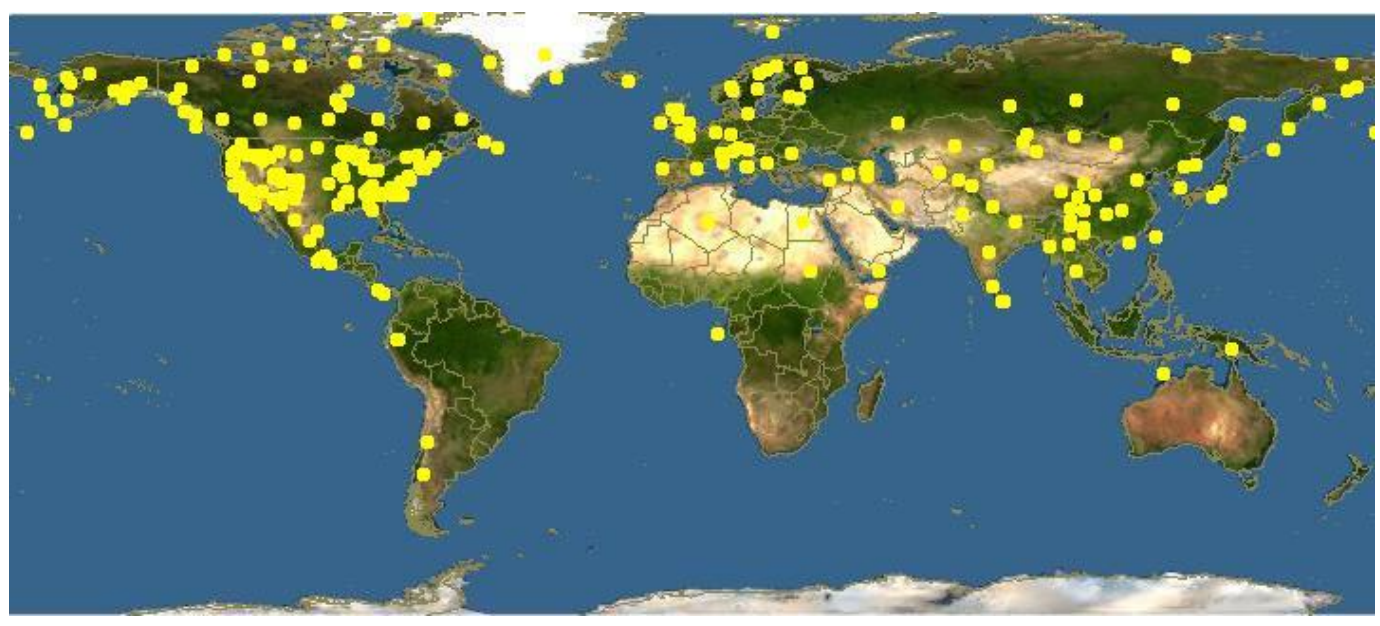

Figure 2. Worldwide distribution of Pedicularis species [source Google images]. 


\section{Phytochemistry}

The genus Pedicularis is a rich source of different secondary metabolites mainly belonging to the polar fraction. In fact, Pedicularis species are poor essential oil producers. Only three species have been investigated as to this aspect, i.e., Pedicularis condensata M.Bieb. (u.n.), P. sibthorpii Boiss. (a.n.), and P. wilhelmsiana Fisch. ex M.Bieb (a.n.). The first one was collected in Turkey and showed the presence of several typical components of essential oils, i.e., more or less oxidized hydrocarbon derivatives and volatile terpenes [10]. The same composition was also observed in the accession of P. wilhelmsiana collected in Iran [11]. Indeed, an important difference was found between the two studied exemplars of P. sibthorpii, both collected in Iran but in two different regions. In fact, the work by Khodaie et al. [12] did not absolutely evidence the presence of sesquiterpenes, while the work by Morteza-Semnani et al. [13] reported these constituents in high amounts, representing $35.4 \%$ of all the identified components. This may actually have been explained by the different environmental growth conditions of the two studied species, which, once again, highlight how essential oil composition is greatly affected by external factors and does not depend only on genetics [14].

Among the polar fraction metabolites, several classes of natural compounds were found, i.e., fatty acids, alkaloids, steroids, lignans, neo-lignans, tannins, ionones, phenylpropanoid glycosides, phenylethanoid glycosides, flavonoids, xanthones, iridoids, seco-iridoids, phenyl-glycosides, organic acids, polyols, saccharides, and amino acids.

Table 1 reports on the components identified in all studied Pedicularis species as reported in literature, according to the species.

As Table 1 clearly shows, only 59 species have been studied for their phytochemical profiles, and, out of these, 12 have been studied only preliminarily, evidencing the presence of some classes of natural compounds but not the specific compounds.

The highest amounts of identified compounds have been recorded in 14 species, i.e., P. artselaeri, P. chinensis, P. decora, P. densispica, P. dolichocymba, P. kansuensis, P. longiflora, P. longiflora var. tubiformis, P. muscicola, P. rex, P. striata, P. torta, P. tricolor, and P. verticillata, while the lowest amounts have been recorded in 6 species, i.e., P. acmodonta, P. bracteosa, P. comosa, P. grayi, P. sarawchanica, and P. semibarbata. All the other species have been shown to biosynthesize metabolites in medium amounts. In only two cases, the data reported in literature have not specified the organs of the plant species that were studied, i.e., P. acmodonta and P. dolichorrhiza.

In general, the studied organs of the plants have been the aerial parts, the leaves, the flowers, or the whole plant, besides a few exceptions, such as P. chinensis and $P$. grayi, where the roots have been analyzed, and P. sarawchanica, where the fruits have been analyzed.

Indeed, for what concerns the other accepted, synonymous, and unresolved named species, no phytochemical data or even no data at allare reported in literature.

Table 2 reports, instead, on the components identified in all the studied Pedicularis species, as reported in literature, according to the compound.

As Table 2 clearly shows, most of the phytochemicals identified in the Pedicularis genus belong to the class of natural metabolites known as iridoids. Phenylethanoid glycosides represent the second major class in this context. On the other hand, only one compound belonging to each of the natural classes of alkanes, fatty acids, and coumarins has been isolated from Pedicularis spp.

The iridoid acucubin and the phenylethanoid glycoside verbascoside are the two most common compounds in the entire genus, whereas some cases of specific compounds evidenced in only one species have also been observed.

As concerns the rest, the presence of other classes of natural metabolites has been shown to be at a medium level, along with their occurrence within the Pedicularis genus. 
Table 1. Phytochemical Compounds Reported in the Studied Pedicularis Species.

\begin{tabular}{|c|c|c|c|}
\hline Pedicularis spp. & Studied Organs & Phytochemical Compounds & References \\
\hline P. acmodonta Boiss. (u.n.) & n.s. & leucosceptoside A, echinacoside & [15] \\
\hline P. alaschanica Maxim. (a.n.) & aerial parts & $\begin{array}{l}\text { alaschanioside A, alaschanioside } \mathrm{C} \text {, citrusin } \mathrm{A} \text {, syringaresinol-4-O- } \beta \text {-D-glucoside, } \\
\text { verbascoside, leucosceptoside } \mathrm{A} \text {, martynoside, boschnaloside, ixoroside, } \\
\text { euphroside, geniposidic acid, mussaenosidic acid }\end{array}$ & {$[16,17]$} \\
\hline P. armata Maxim (a.n.) & whole plant & $\begin{array}{l}\text { armaoside, citrusin B, euphroside, mussaenoside, geniposidic acid, 8-epi-loganic } \\
\text { acid, aucubin }\end{array}$ & [18] \\
\hline P. artselaeri Maxim. (a.n.) & whole plant & $\begin{array}{l}\text { lariciresinol-4-O- } \beta \text {-D-glucoside, lariciresinol-4'-O- } \beta \text {-D-glucoside, alaschanioside A, } \\
\text { citrusin A, artselaeroside A, artselaeroside B, } \\
\text { 2-(p-hydroxyphenyl)-ethanol-1-O- } \beta \text {-D-glucopyranoside, iso-verbascoside, } \\
\text { martynoside, artselaenin I, artselaenin III, artselaenin A, artselaenin B, artselaenin C, } \\
\text { 8-epi-loganic acid, 8-epi-loganin, 7-deoxy-8-epi-loganic acid, plantarenaloside, } \\
\text { mussaenoside, aucubin, 6-O-methyl-aucubin, 6-O-methyl-epi-aucubin, ixoroside, } \\
\text { 7-deoxy-gardoside, gardoside methyl ester, caryoptoside, shanzhiside methyl ester, } \\
\text { 3-methoxy-4-primeverosyl-acetophenone }\end{array}$ & {$[19,20]$} \\
\hline P. bicornuta Klotzsch (u.n.) & whole plant & $\begin{array}{l}\text { alkaloids, lignans glycosides, phenylpropanoid glycosides, flavonoids, iridoids } \\
\text { (exact compounds not specified) }\end{array}$ & [21] \\
\hline P. bracteosa Benth. (a.n.) & aerial parts & aucubin, mussaenoside & [22] \\
\hline $\begin{array}{l}\text { P. bracteosa subsp. paysoniana (Pennell) } \\
\text { W.A. Weber (a.n.) }\end{array}$ & whole plant & alkaloids (exact compounds not specified) & [23] \\
\hline P. capitata Adams (a.n.) & leaves & alkaloids (exact compounds not specified) & [24] \\
\hline P. cephalantha Franch. ex. Maxim. (a.n.) & whole plant & $\begin{array}{l}\text { kidjolanin, pinoresinol, martynoside, iso-martynoside, clerodenoside A, acacetin, } \\
\text { luteolin, } 7 \text {-deoxy-gardoside, plantarenaloside, mussaenosidic acid, euphroside, } \\
\text { mussaenoside, aucubin }\end{array}$ & [25] \\
\hline P. chamissonis Steven (a.n.) & leaves & verbascoside, luteolin-7-O-glucoside, luteolin-7-O-glucuronide & [26] \\
\hline P. chinensis Maxim. (a.n.) & roots & 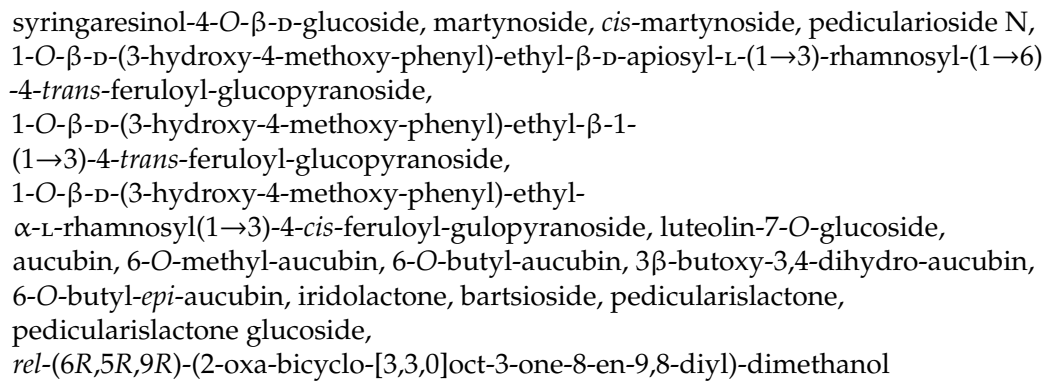 & {$[27,28]$} \\
\hline
\end{tabular}


Table 1. Cont.

\begin{tabular}{ccl}
\hline Pedicularis spp. & Studied Organs & \multicolumn{1}{c}{ Phytochemical Compounds } \\
\hline P. comosa L. (a.n.) & aerial parts & verbascoside, forsythoside B \\
\hline P. condensata & aerial parts & $\begin{array}{l}\text { verbascoside, echinacoside, aucubin, 6-O-acetyl-aucubin, 8-epi-loganin, } \\
\text { mussaenoside, shanzhiside methyl ester, gardoside methyl ester }\end{array}$ \\
\hline P. crenulata Benth. (a.n.) & aerial parts & anagyrine, aucubin, euphroside, plantarenaloside \\
\hline [15] & $\begin{array}{l}\beta \text {-sitosterol, } \beta \text {-daucosterol, iso-verbascoside, kaempferol, aucubin, lamalbid, } \\
\text { pedicularislactone glucoside, ningpogoside B, D-mannitol, } \\
\beta \text {-(3', } 4^{\prime} \text {-dihydroxyphenyl-O-a-L-rhamnopyranosyl- (1-3)- } \beta \text {-D-glucopyranoside, } \\
\text { salicylic acid, 2,5-dihydroxybenzoic acid, 3-hydroxy-4-methoxybenzoic acid, } \\
\text { 3-methoxy-4-hydroxybenzoic acid, aspartic acid, threonine, serine, glutamic acid, } \\
\text { glycine, alanine, cysteine, methionine, isoleucine, phenylalanine, valine, arginine, } \\
\text { proline, leucine, tyrosine }\end{array}$ \\
\hline
\end{tabular}

pedicutricone $\mathrm{A},(+)$-isolariciresinol 3a-O- $\beta$-D-glucopyranoside,

pinoresinol-4-O- $\beta$-D-glucoside, syringaresinol-4-O- $\beta$-D-glucoside, longifloroside B,

densispicoside, verbascoside, martynoside, iso-martynoside,

2 "-O-acetyl-verbascoside, cis-martynoside, salidroside, darendoside B,

P. densispica Franch. ex. Maxim. (a.n.) whole plant

$4-O-\beta$-D-glucopyranosyl-sinapic acid methyl ester,

3-(4-hydroxy-3-methoxyphenyl)-1,2,3-propantriol, citrusin C, robustaside B,

acacetin, kaempferol, apigenin-7-O-glucoside,

kaempferol-3,7-O- $\alpha$-di-rhamnopyranoside, scutellarein-7-O-glucoside,

chrysoeriol-7-O-glucoside, mussaenin A, mussaenoside, argyol, densispicnin A,

densispicnin B, densispicnin C, densispicnin D, shanzhiside methyl ester,

8-epi-loganin, dearabinosyl-pneumonanthoside, maltol- $\beta$-D-glucoside

plantagonine, indicaine, pediculidine, pediculine, lariciresinol- $4^{\prime}-O-\beta$-D-glucoside,

verbascoside, martynoside, $2^{\prime \prime \prime}$-O-acetyl-martynoside, leucosceptoside $\mathrm{A}$, jionoside

$\mathrm{D}, 2$-phenylethyl-O- $\beta$-D-xylopyranosyl-( $(1 \rightarrow 2)-\beta$-D-glucopyranoside, benzyl

P. dolichocymba Hand.-Mazz. (a.n.)

whole plant

alcohol-O- $\beta$-D-xylopyranosyl-( $(\rightarrow 2)-\beta$-D-glucopyranoside, apigenin,

$[30,40-42]$

dolichocymboside $\mathrm{A}$, dolichocymboside $\mathrm{B}$, dolichocymboside $\mathrm{C}$, dolichocymboside

$\mathrm{D}$, gardoside methyl ester, 7-O-acetyl-gardoside methyl ester, uridine, adenosine

\begin{tabular}{ccl}
\hline P. dolichorrhiza Schrenk (a.n.) & n.s. & plantagonine, indicaine, pediculidine, pediculine \\
\hline P. gracilis Wall. ex. Benth. (a.n.) & whole plant & tannins, terpenoids, flavonoids, glycosides (exact compounds not specified) \\
\hline P. grayi A. Nelson (a.n.) & roots & N-methyl-cytisine \\
\hline P. groenlandica Retz. (a.n.) & aerial parts & senecionine, aucubin, euphroside, mussaenoside \\
\hline P. integrifolia Hook. f. (a.n.) & aerial parts & alkaloids, tannins (exact compounds not specified) \\
\hline
\end{tabular}


Table 1. Cont

\begin{tabular}{|c|c|c|c|}
\hline Pedicularis spp. & Studied Organs & Phytochemical Compounds & References \\
\hline P. kanei Durand (s.n.) & leaves & alkaloids (exact compounds not specified) & [24] \\
\hline P. kansuensis Maxim. (a.n.) & whole plant & $\begin{array}{l}\beta \text {-sitosterol, } \beta \text {-daucosterol, 1,2,3,16,19,20-hexahydroxyolean-12-en-28-oic acid, } \\
\text { alaschanioside A, alaschanioside } \mathrm{C}, \text { verbascoside, leucosceptoside A, martynoside, } \\
\text { iso-martynoside, cis-iso-martynoside, } 2^{\prime \prime \prime}, 3^{\prime \prime \prime} \text {-O-diacetyl-martynoside, jionoside B1, } \\
\text { pedicularioside A, pedicularioside } \mathrm{M} \text {, echinacoside, forsythoside B, } \\
\text { phenethylalcohol } \beta \text {-sophoroside, 1-(2,3,4-trihydroxyphenyl)ethyl-3-O-rhamnose-4 } \\
\text {-[(2E)-3-(3,4-dihydroxyphenyl)-2-propenoate]-glucopyranoside, } \\
\text { 1-(2,3,4-trihydroxyphenyl)ethyl-3-O-rhamnose-4-[(2E)-3-(3,4-dihydroxyphenyl)-2 } \\
\text {-propenoate]-6-[(2E)-3-(3,4-dihydroxyphenyl)-2-propenoate]-glucopyranoside, } \\
\text { 4'-methyl-chrysoeriol, luteolin, } \\
\text { luteolin-7-O-glucoside, lagotiside, tricin-7-O-glucuronide, kansuenin, kansuenin B, } \\
\text { kansuenoside, ixoroside, gardoside methyl ester, geniposidic acid, euphroside, } \\
\text { mussaenoside, boschnaloside, 7-deoxy-8-epi-loganic acid, 8-epi-loganic acid, } \\
\text { aucubin, geniposidic acid, (E)-2-hexenyl } \beta \text {-sophoroside, } \\
\text { 3-methoxy-4-hydroxybenzoic acid }\end{array}$ & {$[45-50]$} \\
\hline P. kerneri Dalla Torre (a.n.) & aerial parts & $\begin{array}{l}\text { verbascoside, leucosceptoside A, echinacoside, aucubin, monomelittoside, } \\
\text { plantarenaloside, euphroside, mussaenosidic acid, 8-epi-loganic acid, D-mannitol }\end{array}$ & [51] \\
\hline P. langsdorffii Fisch. ex. Steven (a.n.) & leaves & alkaloids, tannins (exact compounds not specified) & [24] \\
\hline P. lapponica L. (a.n.) & aerial parts & alkaloids (exact compounds not specified), euphroside, aucubin, mussaenoside & {$[24,52]$} \\
\hline P. longiflora Rudolph (a.n.) & whole plant & $\begin{array}{l}\text { scopoletin, longifloroside } \mathrm{A} \text {, longifloroside } \mathrm{B} \text {, longifloroside } \mathrm{C} \text {, longifloroside } \mathrm{D} \text {, } \\
7(R) \text {-dehydro-diconiferyl alcohol-4-O- } \beta \text {-D-glucoside, longiflor } \mathrm{A} \text {, longiflor } \mathrm{B} \text {, } \\
\text { tortoside } \mathrm{D} \text {, tortoside E, verbascoside, iso-verbascoside, leucosceptoside } \mathrm{A} \text {, } \\
\text { pedicularioside } \mathrm{A} \text {, pedicularoside I, pedicularoside } \mathrm{M} \text {, cistanoside } \mathrm{D} \text {, echinacoside, } \\
\text { geniposidic acid, mussaenoside, loganic acid, longifloroside, adenosine, } \\
6-\left(1^{\prime \prime}, 3^{\prime \prime} \text {-dihydroxy-2"-propoxyl)-inosine }\right.\end{array}$ & {$[47,54-56]$} \\
\hline $\begin{array}{l}\text { P. longiflora var. tubiformis (Klotzsch) } \\
\text { Tsoong (a.n.) }\end{array}$ & whole plant & $\begin{array}{l}\text { hexatriacontanol, nonatriacontanol, 1-hydroxy-xanthone, } \beta \text {-daucosterol, } \\
\text { martynoside, apigenin, chrysoeriol, luteolin, tricin, acacetin, orientin, morelosin, } \\
\text { apigenin-7-O-glucuronide, } \\
\text { luteolin-7-O-glucoside, luteolin-5-O-glucoside, chrysoeriol-7-O-glucuronide, } \\
\text { luteolin-7-O-glucuronide, tricin-7-O-glucuronide, 7-deoxy-8-epi-loganic acid, } \\
\text { mussaenosidic acid, boschnaloside, aucubin, muconic acid, cinnamic acid, } p \text {-formyl } \\
\text { cinnamic acid }\end{array}$ & [57-59] \\
\hline
\end{tabular}


Table 1. Cont.

\begin{tabular}{|c|c|c|c|}
\hline Pedicularis spp. & Studied Organs & Phytochemical Compounds & References \\
\hline P. muscicola Maxim. (a.n.) & whole plant & $\begin{array}{l}\text { hentriacontane, arachidic acid, } \beta \text {-daucosterol, syringaresinol-4-O- } \beta \text {-D-glucoside, } \\
\text { verbascoside, martynoside, cis-martynoside, pedicularioside A, mussaenoside, } \\
\text { euphroside, geniposidic acid, aucubin, mussaenosidic acid, shanzhiside methyl } \\
\text { ester, penstemonoside, pedicularioside, gardoside methyl ester, sesamoside, } \\
\text { phloyoside II, caryoptoside, D-mannitol }\end{array}$ & [60-62] \\
\hline P. nordmanniana Bunge (u.n.) & aerial parts & $\begin{array}{l}\text { verbascoside, martynoside, leucosceptoside A, forsythoside B, iridolactone, } \\
\text { geniposidic acid, aucubin, euphroside, mussaenoside }\end{array}$ & [63] \\
\hline P. palustris $\mathrm{L}$. & aerial parts & $\begin{array}{l}\text { aucubin, euphroside, ixoroside, shanzhiside methyl ester, gardoside methyl ester, } \\
\text { plantarenaloside, mussaenoside, pedicularioside, penstemonoside, boschnaloside, } \\
\text { 8-epi-loganin, 7-deoxy-8-epi-loganin, 8-epi-loganic acid }\end{array}$ & [52] \\
\hline P. pectinata Wall. ex. Benn. (a.n.) & flowers & phenolics (exact compounds not specified) & [21] \\
\hline P. peduncularis Popov (a.n.) & aerial parts & plantagonine, indicainine, plantagonin, indicine, peducularine, $N$-methyl-cytisine & [64] \\
\hline P. plicata Maxim.(a.n.) & whole plant & $\begin{array}{l}\text { verbascoside, martynoside, iso-martynoside, cis-leucosceptoside A, } \\
\text { 3,4-dihydroxy-phenethyl alcohol, } \\
\text { 1-O- } \beta \text {-D-( } 3,4 \text {-dihydroxy- } \beta \text {-phenylethyl)-glucopyranoside, boschnaloside, } \\
\text { plicatoside A, plicatoside B }\end{array}$ & [65] \\
\hline P. procera A. Gray (u.n.) & aerial parts & $\begin{array}{l}\text { aucubin, mussaenoside, 6-deoxy-catalpol, shanzhiside methyl ester, 8-epi-loganic } \\
\text { acid, gardoside, proceroside }\end{array}$ & {$[22,66]$} \\
\hline P. pycnantha Boiss. (u.n.) & whole plant & alkaloids, tannins (exact compounds not specified) & [68] \\
\hline P. racemosa Douglas ex. Benth. (a.n.) & aerial parts & lupanine, tetrahydrorhombifoline, aucubin, euphroside & {$[22,30]$} \\
\hline P. resupinata L. (a.n.) & whole plant & $\begin{array}{l}\text { alaschanioside } \mathrm{A} \text {, alaschanioside } \mathrm{C} \text {, syringaresinol-4" } 4^{\prime \prime}-\mathrm{O}-\beta \text {-D-glucoside, } \\
\text { verbascoside, } 2^{\prime \prime \prime}, 3^{\prime \prime \prime} \text {-O-diacetyl-martynoside, leucosceptoside } \mathrm{A} \text {, plantarenaloside, } \\
\text { euphroside, boschnaloside, gardoside methyl ester, geniposidic acid }\end{array}$ & {$[16,49]$} \\
\hline P. rex C.B. Clarke ex. Maxim. (a.n.) & whole plant & $\begin{array}{l}\text { verbascoside, martynoside, iso-martynoside, } \\
\text { 4-hydroxy-phenylpropenyl- } \alpha \text {-L-rhamnopyranosyl-( } 1 \rightarrow 3)-4 \text {-O-feruloyl- } \beta \text {-D-glucopyranoside, } \\
\text { apigenin, chrysoeriol, luteolin, luteolin-7-O-glucoside, } \\
5,4^{\prime} \text {-di-hydroxy-3'-methoxy-flavone-7-O- } 6^{\prime \prime}-n \text {-butyryl- } \beta \text {-D-glucopyranoside, } \\
\text { aucubin, 6-O-ethyl-aucubin, euphroside, 6-O-ethyl-epi-aucubin, mussaenoside, } \\
\text { plantarenaloside, pedicurexoside }\end{array}$ & [69] \\
\hline P. rostratocapitata Crantz (a.n.) & aerial parts & $\begin{array}{l}\text { verbascoside, echinacoside, campneoside II, cistantubuloside } \mathrm{C}_{1} \text {, aucubin, } \\
\text { euphroside, monomelittoside, mussaenosidic acid, 8-epi-loganic acid }\end{array}$ & [70] \\
\hline
\end{tabular}


Table 1. Cont

\begin{tabular}{|c|c|c|c|}
\hline Pedicularis spp. & Studied Organs & Phytochemical Compounds & References \\
\hline P. sarawschanica Regel (u.n.) & fruits & plantagonine, peducularine & [71] \\
\hline P. semibarbata A. Gray (a.n.) & whole plant & $\alpha$-iso-lupanine, 17-oxo-iso-lupanine or isomer & [72] \\
\hline P. semitorta Maxim. (a.n.) & whole plant & $\begin{array}{l}\text { syringaresinol- } 4^{\prime \prime}-\mathrm{O}-\beta \text {-D-glucoside, semitortoside A, semitortoside B, cis-iso-verbascoside, } \\
\text { shanzhiside methyl ester, mussaenoside }\end{array}$ & [73] \\
\hline P. sibthorpii & aerial parts & verbascoside, martynoside, iso-martynoside, luteolin 7-O-glucoside, aucubin, D-mannitol & [74] \\
\hline P. siphonantha D.Don (a.n.) & whole plant & $\begin{array}{l}\text { (+)-dehydro-vomifoliol, vomifoliol, w-hydroxy-propioguaiacone, } \\
\text { 3-hydroxy-1-(4-hydroxy-3,5-dimethoxyphenyl)-1-propanone }\end{array}$ & [25] \\
\hline P. spicata Pall. (a.n.) & whole plant & $\begin{array}{l}\text { verbascoside, permethyl-verbascoside, pedicularioside A, pedicularioside G, pedicularioside } \\
\text { H, cis-pedicularioside H, shanzhiside methyl ester, gardoside methyl ester, } \\
\text { 5-deoxy-puchelloside I }\end{array}$ & {$[75,76]$} \\
\hline P. striata Pall. (a.n.) & whole plant & $\begin{array}{l}\text { ecdysterone 3-O- } \beta \text {-D-galactoside, striatoside } \mathrm{A} \text {, striatoside } \mathrm{B} \text {, verbascoside, iso-verbascoside, } \\
\text { echinacoside, pedicularioside A, pedicularioside } \mathrm{G} \text {, pedicularioside } \mathrm{H} \text {, } \\
\text { decaffeoyl-verbascoside, } 1^{\prime}-O-\beta \text {-D- } \\
\text { (3-methoxy-4-hydroxy-phenyl)-ethyl- } \alpha \text {-L-apiosyl- }\left(1 \rightarrow 3^{\prime}\right)-\alpha \text {-L } \\
\text {-rhamnosyl- }\left(1 \rightarrow 6^{\prime}\right)-4^{\prime} \text {-cis-feruloyl-glucopyranoside, } 8 \text {-O-acetyl-harpagide, } \\
\text { dihydro-catalpolgenin }\end{array}$ & [77-79] \\
\hline $\begin{array}{l}\text { P. striata subsp. aracnoidea (Franch.) } \\
\text { Tsoong }\end{array}$ & whole plant & $\begin{array}{l}\text { verbascoside, pedicularioside } \mathrm{A} \text {, pedicularioside } \mathrm{M} \text {, pedicularioside } \mathrm{N} \text {, } \\
\text { dihydro-catalpolgenin, eremophila-10,11-dien-7a,13-diol }\end{array}$ & [80-82] \\
\hline P. sudetica Willd. (a.n.) & leaves & alkaloids (exact compounds not specified) & [24] \\
\hline P. sylvatica L.(a.n.) & aerial parts & luteolin-7-O-glucoside, euphroside, plantarenaloside, 8-epi-loganin & {$[11,52]$} \\
\hline P. tenuirostris Benth. (a.n.) & flowers and leaves & phenolics (exact compounds not specified) & [8] \\
\hline P. torta Maxim. (a.n.) & whole plant & $\begin{array}{l}\text { longiflor } \mathrm{A} \text {, longiflor } \mathrm{B} \text {, tortoside } \mathrm{A} \text {, tortoside } \mathrm{B} \text {, tortoside } \mathrm{C} \text {, tortoside } \mathrm{D} \text {, tortoside } \mathrm{E} \text {, } \\
\text { tortoside } \mathrm{F} \text {, dihydro-dehydro-diconiferyl alcohol-4- } O \text { - } \alpha \text {-L-rhamnoside, } \\
\text { dihydro-dehydro-diconiferyl alcohol-4-O- } \beta \text {-D-glucoside, dihydro-dehydro-diconiferyl } \\
\text { alcohol-9-O- } \beta \text {-D-glucoside, ( }(7 R) \text {-dehydro-diconiferyl alcohol-4-O- } \beta \text {-D-glucoside, } \\
\text { (7S)-dehydro-diconiferyl alcohol-4- } O-\beta \text {-D-glucoside, verbascoside, leucosceptoside A, } \\
\text { cistanoside D, shanzhiside methyl ester, gardoside methyl ester, } 8 \text {-epi-loganin, loganic acid }\end{array}$ & {$[83,84]$} \\
\hline
\end{tabular}


Table 1. Cont

\begin{tabular}{|c|c|c|c|}
\hline Pedicularis spp. & Studied Organs & Phytochemical Compounds & References \\
\hline P. tricolor Hand.-Mazz. (a.n.) & whole plant & 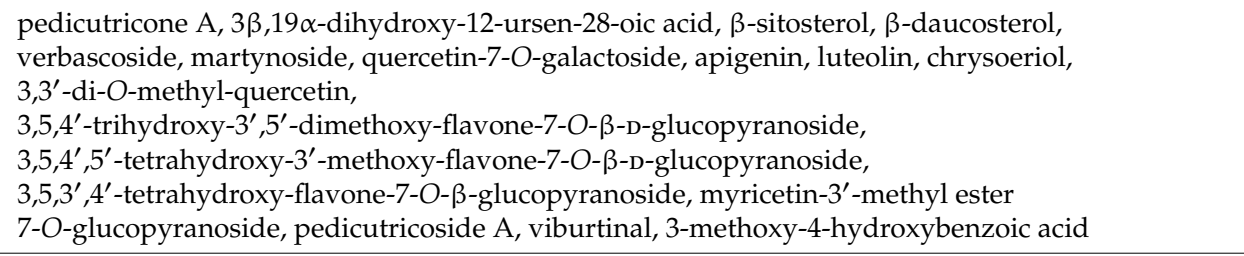 & [85] \\
\hline P. uliginosa Bunge (a.n.) & whole plant & $\begin{array}{l}\text { (rel-4aS,7R,7aR)-1,4a,5,6,7,7a-hexahydro-7-hydroxyl-7-methyl-cyclopenta[c]pyran-4-carboxaldehyde, } \\
\text { 1,3,5,6-tetrahydro-1-methoxyl-7-methyl-cyclopenta[c]pyran-4-carboxaldehyde, } \\
\text { (rel-1R,4S,4aS,7R,7aR)-7-methyl-hexahydro-1,4-(epoxymethano)-cyclopenta[c]pyran-3(1H)-one, } \\
\text { 4-epi-alyxialactone, alyxialactone, artselaenin A, artselaenin B, boschnarol, } \\
\text { (4R)-4-hydroxymethyl- boschnialactone, densispicnin B }\end{array}$ & [86] \\
\hline P. verticillata L. (a.n.) & whole plant & $\begin{array}{l}\text { verticillatoside A, verticillatoside B, verbascoside, leucosceptoside A, cistanoside D, } \\
\text { echinacoside, angoroside A, cistantubuloside } B_{1} \text {, wiedemannioside } C \text {, excelside B, aucubin, } \\
\text { euphroside, monomelittoside, mussaenosidic acid, 7-deoxy-8-epi-loganic acid, 8-epi-loganic } \\
\text { acid, plantarenaloside, geniposidic acid, boschnaloside, caryoptoside, pediverticilatasin A, } \\
\text { pediverticilatasin B, pediverticilatasin C, kansuenin B, densispicnin B, euphrasin, scyphiphin } \\
\text { A1, scyphiphin A2, ligustroside }\end{array}$ & {$[70,87,88]$} \\
\hline P. wilhelmsiana & aerial parts & phenolics (exact compounds not specified) & [12] \\
\hline
\end{tabular}

Table 2. Distribution of the Phytochemicals in the Various Pedicularis Species.

\begin{tabular}{cccc}
\hline Phytochemical Class & Phytochemical Compound & Pedicularis spp. & References \\
\hline Alkanes & hentriacontane & P. muscicola \\
\hline Alkyl alcohols & hexatriacontanol & P. longiflora var. tubiformis & P. longiflora var. tubiformis \\
\cline { 2 - 3 } & nonatriacontanol & P. muscicola & [58,59] \\
\hline Fatty acids & arachidic acid & P. longiflora & [62] \\
\hline Coumarins & scopoletin & P. longiflora var. tubiformis & P. densispica \\
Panthones & 1-hydroxy-xanthone & [58,59] \\
\hline
\end{tabular}


Table 2. Cont.

\begin{tabular}{|c|c|c|c|}
\hline Phytochemical Class & Phytochemical Compound & Pedicularis spp. & References \\
\hline \multirow{6}{*}{ Terpenoids } & 1,2,3,16,19,20-hexahydroxyolean-12-en-28-oic acid & P. kansuensis & {$[50]$} \\
\hline & $3 \beta, 19 \alpha$-dihydroxy-12-ursen-28-oic acid & P. tricolor & [85] \\
\hline & $\beta$-sitosterol & $\begin{array}{l}\text { P. decora } \\
\text { P. kansuensis } \\
\text { P. tricolor }\end{array}$ & {$[32-36,45,85]$} \\
\hline & $\beta$-daucosterol & $\begin{array}{c}\text { P. decora } \\
\text { P. kansuensis } \\
\text { P. longiflora var. tubiformis } \\
\text { P. muscicola } \\
\text { P. tricolor }\end{array}$ & {$[32-36,45,58,59,62,85]$} \\
\hline & ecdysterone 3-O- $\beta$-D-galactoside & P. striata & [78] \\
\hline & kidjolanin & P. cephalantha & [25] \\
\hline \multirow{10}{*}{ Alkaloids } & $\alpha$-iso-lupanine & P. semibarbata & [72] \\
\hline & anagyrine & P. crenulata & {$[30,42]$} \\
\hline & indicaine & $\begin{array}{l}\text { P. dolichorrhiza } \\
\text { P. dolichocymba }\end{array}$ & {$[30,42]$} \\
\hline & indicainine & P. peduncularis & [64] \\
\hline & indicine & P. peduncularis & [64] \\
\hline & lupanine & P. racemosa & {$[30]$} \\
\hline & $N$-methyl-cytisine & $\begin{array}{c}\text { P. grayi } \\
\text { P. peduncularis }\end{array}$ & {$[30,64]$} \\
\hline & peducularine & $\begin{array}{l}\text { P. peduncularis } \\
\text { P. sarawschanica }\end{array}$ & {$[64,71]$} \\
\hline & pediculidine & $\begin{array}{l}\text { P. dolichocymba } \\
\text { P. dolichorrhiza }\end{array}$ & {$[30,42]$} \\
\hline & pediculine & $\begin{array}{l}\text { P. dolichocymba } \\
\text { P. dolichorrhiza }\end{array}$ & {$[30,42]$} \\
\hline
\end{tabular}


Table 2. Cont.

\begin{tabular}{|c|c|c|c|}
\hline Phytochemical Class & Phytochemical Compound & Pedicularis spp. & References \\
\hline & plantagonin & P. peduncularis & [64] \\
\hline & plantagonine & $\begin{array}{l}\text { P. dolichocymba } \\
\text { P. dolichorrhiza } \\
\text { P. peduncularis } \\
\text { P. sarawschanica }\end{array}$ & {$[30,42,64,71]$} \\
\hline & senecionine & P. groenlandica & [30] \\
\hline & tetrahydrorhombifoline & P. racemosa & {$[30]$} \\
\hline \multirow{11}{*}{ Lignans and neo-lignans } & $7(R)$-dehydro-diconiferyl alcohol-4-O- $\beta$-D-glucoside & $\begin{array}{l}\text { P. longiflora } \\
\text { P. torta }\end{array}$ & {$[54,83]$} \\
\hline & (7S)-dehydro-diconiferyl alcohol-4-O- $\beta$-D-glucoside & P. torta & [84] \\
\hline & $(+)$-isolariciresinol 3a-O- $\beta$-D-glucopyranoside & P. densispica & [39] \\
\hline & alaschanioside A & $\begin{array}{l}\text { P. alaschanica } \\
\text { P. artselaeri } \\
\text { P. kansuensis } \\
\text { P. resupinata }\end{array}$ & {$[17,19,47,49,50]$} \\
\hline & alaschanioside C & $\begin{array}{l}\text { P. alaschanica } \\
\text { P. kansuensis } \\
\text { P. resupinata }\end{array}$ & {$[17,47,49,50]$} \\
\hline & armaoside & P. armata & [18] \\
\hline & citrusin $\mathrm{A}$ & $\begin{array}{l}\text { P. alaschanica } \\
\text { P. artselaeri }\end{array}$ & {$[17,19]$} \\
\hline & citrusin B & P. armata & {$[17,18]$} \\
\hline & densispicoside & P. densispica & [39] \\
\hline & $\begin{array}{l}\text { dihydro-dehydro-diconiferyl } \\
\text { alcohol-4-O- } \beta \text {-D-glucoside }\end{array}$ & P. torta & [84] \\
\hline & $\begin{array}{l}\text { dihydro-dehydro-diconiferyl } \\
\text { alcohol-4- } O-\alpha \text {-L-rhamnoside }\end{array}$ & P. torta & [84] \\
\hline
\end{tabular}


Table 2. Cont.

\begin{tabular}{|c|c|c|c|}
\hline Phytochemical Class & Phytochemical Compound & Pedicularis spp. & References \\
\hline & $\begin{array}{l}\text { dihydro-dehydro-diconiferyl } \\
\text { alcohol-9-O- } \beta \text {-D-glucoside }\end{array}$ & P. torta & [84] \\
\hline & lariciresinol-4-O- $\beta$-D-glucoside & P. artselaeri & [19] \\
\hline & lariciresinol-4'-O- $\beta$-D-glucoside & $\begin{array}{c}\text { P. artselaeri } \\
\text { P. dolichocymba }\end{array}$ & {$[19,41]$} \\
\hline & longiflor A & $\begin{array}{l}\text { P. longiflora } \\
\text { P. torta }\end{array}$ & {$[54,83]$} \\
\hline & longiflor B & $\begin{array}{l}\text { P. longiflora } \\
\text { P. torta }\end{array}$ & {$[54,83]$} \\
\hline & longifloroside A & P. longiflora & [54] \\
\hline & longifloroside B & $\begin{array}{l}\text { P. densispica } \\
\text { P. longiflora }\end{array}$ & {$[39,54]$} \\
\hline & longifloroside C & P. longiflora & [54] \\
\hline & longifloroside D & P. longiflora & [54] \\
\hline & pinoresinol & P. cephalantha & [25] \\
\hline & semitortoside A & P. semitorta & [73] \\
\hline & semitortoside B & P. semitorta & [73] \\
\hline & pinoresinol-4- $O-\beta$-D-glucoside & P. densispica & [39] \\
\hline & striatoside A & P. striata & [78] \\
\hline & striatoside B & P. striata & [78] \\
\hline & syringaresinol-4-O- $\beta$-D-glucoside & $\begin{array}{l}\text { P. alaschanica } \\
\text { P. chinensis } \\
\text { P. densispica } \\
\text { P. muscicola }\end{array}$ & {$[17,28,39,60,61]$} \\
\hline & syringaresinol- $4^{\prime \prime}-O-\beta-\mathrm{D}$-glucoside & $\begin{array}{l}\text { P. resupinata } \\
\text { P. semitorta }\end{array}$ & {$[49,73]$} \\
\hline & tortoside A & P. torta & [84] \\
\hline & tortoside B & P. torta & [84] \\
\hline & tortoside C & P. torta & [84] \\
\hline
\end{tabular}


Table 2. Cont

\begin{tabular}{|c|c|c|c|}
\hline Phytochemical Class & Phytochemical Compound & Pedicularis spp. & References \\
\hline & tortoside D & $\begin{array}{l}\text { P. longiflora } \\
\text { P. torta }\end{array}$ & {$[54,84]$} \\
\hline & tortoside $\mathrm{E}$ & $\begin{array}{l}\text { P. longiflora } \\
\text { P. torta }\end{array}$ & {$[54,84]$} \\
\hline & tortoside F & P. torta & [84] \\
\hline & verticillatoside $\mathrm{A}$ & P. verticillata & [88] \\
\hline & verticillatoside B & P. verticillata & [88] \\
\hline & $\begin{array}{l}\text { 1-(2,3,4-trihydroxyphenyl)ethyl-3-O-rhamnose-4-[(2E)-3- } \\
\text { (3,4-dihydroxyphenyl)-2-propenoate]-glucopyranoside }\end{array}$ & P. kansuensis & {$[47,49,50]$} \\
\hline & $\begin{array}{l}\text { 1-(2,3,4-trihydroxyphenyl)ethyl-3-O-rhamnose-4-[(2E)-3 } \\
\text {-(3,4-dihydroxyphenyl)-2-propenoate]-6-[(2E)-3- } \\
\text { (3,4-dihydroxyphenyl)-2-propenoate]-glucopyranoside }\end{array}$ & P. kansuensis & {$[47,49,50]$} \\
\hline & 1-O- $\beta$-D-(3,4-dihydroxy- $\beta$-phenylethyl)-glucopyranoside & P. plicata & [65] \\
\hline & $\begin{array}{c}\text { 1-O- } \beta \text {-D-(3-hydroxy-4-methoxy-phenyl)-ethyl- } \\
\beta \text {-D-apiosyl-L-( }(1 \rightarrow 3) \text {-rhamnosyl-( } 1 \rightarrow 6) \text { - } \\
\text { 4-trans-feruloyl-glucopyranoside }\end{array}$ & P. chinensis & [28] \\
\hline & 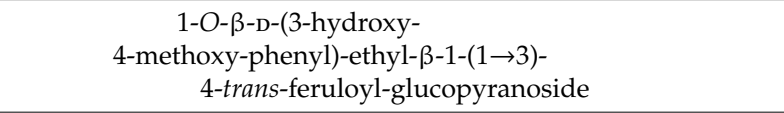 & P. chinensis & [28] \\
\hline & $\begin{array}{l}\text { 1-O- } \beta \text {-D-(3-hydroxy-4-methoxy-phenyl)-ethyl- } \\
\alpha \text {-L-rhamnosyl( } 1 \rightarrow 3 \text { - } \\
\text { 4-cis-feruloyl-gulopyranoside }\end{array}$ & P. chinensis & [28] \\
\hline \multirow{10}{*}{ Phenylethanoid glycosides } & $\begin{array}{l}\text { 1'-O- } \beta \text {-D-(3-methoxy-4-hydroxy-phenyl)-ethyl- } \\
\alpha \text {-L-apiosyl-( }\left(1 \rightarrow 3^{\prime}\right)-\alpha \text {-L-rhamnosyl- } \\
\quad\left(1 \rightarrow 6^{\prime}\right)-4^{\prime} \text {-cis-feruloyl-glucopyranoside }\end{array}$ & P. striata & [78] \\
\hline & 2-(p-hydroxyphenyl)-ethanol-1-O- $\beta$-D-glucopyranoside & P. artselaeri & [19] \\
\hline & 2-phenylethyl-O- $\beta$-D-xylopyranosyl-( $1 \rightarrow 2)$ - $\beta$-D-glucopyranoside & P. dolichocymba & [41] \\
\hline & 2"-O-acetyl-verbascoside & P. densispica & [39] \\
\hline & $2^{\prime \prime \prime}$-O-acetyl-martynoside & P. dolichocymba & [41] \\
\hline & $2^{\prime \prime \prime}, 3^{\prime \prime \prime}$-O-diacetyl-martynoside & $\begin{array}{l}\text { P. kansuensis } \\
\text { P. resupinata }\end{array}$ & {$[47,49,50]$} \\
\hline & 3,4-dihydroxy-phenethyl alcohol & P. plicata & [65] \\
\hline & 3-(4-hydroxy-3-methoxyphenyl)-1,2,3-propantriol & P. densispica & [39] \\
\hline & $\begin{array}{l}\text { 4-hydroxy-phenylpropenyl- } \alpha \text {-L-rhamnopyranosyl-( } 1 \rightarrow 3)-4 \\
\text {-O-feruloyl- } \beta \text {-D-glucopyranoside }\end{array}$ & P. rex & [69] \\
\hline & 4-O- $\beta$-D-glucopyranosyl-sinapic acid methyl ester & P. densispica & [39] \\
\hline
\end{tabular}


Table 2. Cont.

\begin{tabular}{|c|c|c|c|}
\hline Phytochemical Class & Phytochemical Compound & Pedicularis spp. & References \\
\hline & angoroside $\mathrm{A}$ & P. verticillata & [70] \\
\hline & artselaeroside A & P. artselaeri & [20] \\
\hline & artselaeroside B & P. artselaeri & [20] \\
\hline & $\begin{array}{c}\text { benzyl } \\
\text { alcohol-O- } \beta \text {-D-xylopyranosyl-( }(1 \rightarrow 2)-\beta \text {-D-glucopyranoside }\end{array}$ & P. dolichocymba & [41] \\
\hline & cis-iso-martynoside & P. kansuensis & {$[47,49,50]$} \\
\hline & cis-iso-verbascoside & P. semitorta & [73] \\
\hline & cis-leucosceptoside A & P. plicata & {$[65]$} \\
\hline & cis-martynoside & $\begin{array}{l}\text { P. chinensis } \\
\text { P. densispica } \\
\text { P. muscicola }\end{array}$ & {$[28,39,62]$} \\
\hline & cis-pedicularioside $\mathrm{H}$ & P. spicata & [76] \\
\hline & campneoside II & P. rostratocapitata & [70] \\
\hline & cistanoside D & $\begin{array}{l}\text { P. lasiophrys } \\
\text { P. longiflora } \\
\text { P. torta } \\
\text { P. verticillata }\end{array}$ & {$[53,55,84,88]$} \\
\hline & cistantubuloside $B_{1}$ & P. verticillata & [70] \\
\hline & cistantubuloside $C_{1}$ & P. rostratocapitata & [70] \\
\hline & citrusin $\mathrm{C}$ & P. densispica & [39] \\
\hline & clerodenoside A & P. cephalantha & [25] \\
\hline & darendoside B & P. densispica & [39] \\
\hline & decaffeoyl-verbascoside & P. striata & [77] \\
\hline & forsythoside B & $\begin{array}{c}\text { P. comosa } \\
\text { P. kansuensis } \\
\text { P. nordmanniana }\end{array}$ & {$[15,47,49,50,63$} \\
\hline
\end{tabular}


Table 2. Cont.

\begin{tabular}{|c|c|c|c|}
\hline Phytochemical Class & Phytochemical Compound & Pedicularis spp. & References \\
\hline & echinacoside & $\begin{array}{c}\text { P. acmodonta } \\
\text { P. condensata } \\
\text { P. kansuensis } \\
\text { P. kerneri } \\
\text { P. longiflora } \\
\text { P. rostratocapitata } \\
\text { P. striata } \\
\text { P. verticillata }\end{array}$ & {$[15,29,47,49-51,56,70,77]$} \\
\hline & excelside B & P. verticillata & {$[70]$} \\
\hline & iso-martynoside & $\begin{array}{l}\text { P. cephalantha } \\
\text { P. densispica } \\
\text { P. kansuensis } \\
\text { P. plicata } \\
\text { P. rex } \\
\text { P. sibthorpii }\end{array}$ & {$[25,47,49,50,65,69,74]$} \\
\hline & iso-verbascoside & $\begin{array}{l}\text { P. artselaeri } \\
\text { P. decora } \\
\text { P. longiflora } \\
\text { P. striata }\end{array}$ & {$[20,33,47,56,77]$} \\
\hline & jionoside B1 & P. kansuensis & {$[47,49,50]$} \\
\hline & jionoside D & P. dolichocymba & [41] \\
\hline & leucosceptoside A & $\begin{array}{l}\text { P. acmodonta } \\
\text { P. alaschanica } \\
\text { P. dolichocymba } \\
\text { P. kansuensis } \\
\text { P. kerneri } \\
\text { P. lasiophrys } \\
\text { P. longiflora } \\
\text { P. nordmanniana } \\
\text { P. resupinata } \\
\text { P. torta } \\
\text { P. verticillata }\end{array}$ & $\begin{array}{c}{[15,17,41,45,47,49-51,53,} \\
55,63,84,88]\end{array}$ \\
\hline
\end{tabular}


Table 2. Cont.

\begin{tabular}{|c|c|c|c|}
\hline Phytochemical Class & Phytochemical Compound & Pedicularis spp. & References \\
\hline & martynoside & $\begin{array}{c}\text { P. alaschanica } \\
\text { P. artselaeri } \\
\text { P. cephalantha } \\
\text { P. chinensis } \\
\text { P. densispica } \\
\text { P. dolichocymba } \\
\text { P. kansuensis } \\
\text { P. longiflora var. tubiformis } \\
\text { P. muscicola } \\
\text { P. nordmanniana } \\
\text { P. plicata } \\
\text { P. rex } \\
\text { P. sibthorpii } \\
\text { P. tricolor }\end{array}$ & $\begin{array}{c}{[17,20,25,28,39,41,45,47,} \\
49,50,57,62,63,65,69,74,85]\end{array}$ \\
\hline & pedicularioside $\mathrm{A}$ & $\begin{array}{c}\text { P. kansuensis } \\
\text { P. longiflora } \\
\text { P. muscicola } \\
\text { P. spicata } \\
\text { P. striata } \\
\text { P. striata subsp. aracnoidea }\end{array}$ & {$[47,49,50,56,62,75,77,82]$} \\
\hline & pedicularioside $\mathrm{E}$ & P. lasiophrys & [53] \\
\hline & pedicularioside $\mathrm{G}$ & $\begin{array}{l}\text { P. spicata } \\
\text { P. striata }\end{array}$ & {$[76,79]$} \\
\hline & pedicularioside $\mathrm{H}$ & $\begin{array}{l}\text { P. spicata } \\
\text { P. striata }\end{array}$ & {$[75,78]$} \\
\hline & pedicularioside I & P. longiflora & [55] \\
\hline & pedicularioside $\mathrm{M}$ & $\begin{array}{c}\text { P. kansuensis } \\
\text { P. longiflora } \\
\text { P. striata subsp. aracnoidea }\end{array}$ & {$[47,49,50,56,82]$} \\
\hline & pedicularioside $\mathrm{N}$ & $\begin{array}{c}\text { P. chinensis } \\
\text { P. striata subsp. aracnoidea }\end{array}$ & {$[28,82]$} \\
\hline & permethyl-verbascoside & P. spicata & [76] \\
\hline
\end{tabular}


Table 2. Cont.

\begin{tabular}{|c|c|c|c|}
\hline Phytochemical Class & Phytochemical Compound & Pedicularis spp. & References \\
\hline & phenethylalcohol $\beta$-sophoroside & P. kansuensis & [45] \\
\hline & robustaside B & P. densispica & [39] \\
\hline & salidroside & P. densispica & [39] \\
\hline & verbascoside & $\begin{array}{c}\text { P. alaschanica } \\
\text { P. chamissonis } \\
\text { P. comosa } \\
\text { P. condensata } \\
\text { P. densispica } \\
\text { P. dolichocymba } \\
\text { P. kansuensis } \\
\text { P. kerneri } \\
\text { P. lasiophrys } \\
\text { P. longiflora } \\
\text { P. muscicola } \\
\text { P. nordmanniana } \\
\text { P. plicata } \\
\text { P. punctata } \\
\text { P. resupinata } \\
\text { P. rex } \\
\text { P. rostratocapitata } \\
\text { P. sibthorpii } \\
\text { P. spicata } \\
\text { P. striata } \\
\text { P. striata subsp. aracnoidea } \\
\text { P. tricolor } \\
\text { P. torta } \\
\text { P. verticillata }\end{array}$ & $\begin{array}{c}{[8,15,17,26,29,39,41,45,47,} \\
49-51,53,55,62,63,65,69,70 \\
74,75,77,82,84,85,88]\end{array}$ \\
\hline & wiedemannioside C & P. verticillata & {$[70]$} \\
\hline & $3,3^{\prime}$-di-O-methyl-quercetin & P. tricolor & [85] \\
\hline & $\begin{array}{c}\text { 3,5,4'-trihydroxy-3', } 5^{\prime} \text {-dimethoxy-flavone- } \\
\text { 7-O- } \beta \text {-D-glucopyranoside }\end{array}$ & P. tricolor & [85] \\
\hline & $\begin{array}{c}\text { 3,5,4', } 5^{\prime} \text {-tetrahydroxy-3'-methoxy-flavone- } \\
\text { 7-O- } \beta \text {-D-glucopyranoside }\end{array}$ & P. tricolor & [85] \\
\hline
\end{tabular}


Table 2. Cont.

\begin{tabular}{|c|c|c|c|}
\hline Phytochemical Class & Phytochemical Compound & Pedicularis spp. & References \\
\hline & $\begin{array}{c}\text { 3,5,3', } \text {, }^{\prime} \text {-tetrahydroxy-flavone-7-O- } \\
\beta \text {-gluopyranoside }\end{array}$ & P. tricolor & [85] \\
\hline & $4^{\prime}$-methyl-chrysoeriol & P. kansuensis & [45] \\
\hline & $\begin{array}{l}\text { 5,4'-di-hydroxy- } 3^{\prime} \text {-methoxy-flavone-7-O- } \\
6^{\prime \prime} \text {-n-butyryl- } \beta \text {-D-glucopyranoside }\end{array}$ & P. rex & [69] \\
\hline & acacetin & $\begin{array}{c}\text { P. cephalantha } \\
\text { P. densispica } \\
\text { P. longiflora var. tubiformis }\end{array}$ & {$[25,38,57]$} \\
\hline & apigenin & $\begin{array}{c}\text { P. dolichocymba } \\
\text { P. longiflora var. tubiformis } \\
\text { P. rex } \\
\text { P. tricolor }\end{array}$ & {$[41,57,69,85]$} \\
\hline & apigenin-7-O-glucoside & P. densispica & [38] \\
\hline & apigenin-7-O-glucuronide & P. longiflora var. tubiformis & [57] \\
\hline & chrysoeriol & $\begin{array}{c}\text { P. longiflora var. tubiformis } \\
\text { P. rex } \\
\text { P. tricolor }\end{array}$ & {$[57,69,85]$} \\
\hline & chrysoeriol-7-O-glucoside & P. densispica & [38] \\
\hline & chrysoeriol-7-O-glucuronide & P. longiflora var. tubiformis & [57] \\
\hline & kaempferol & $\begin{array}{l}\text { P. decora } \\
\text { P. densispica }\end{array}$ & {$[32-36,38]$} \\
\hline & kaempferol-3,7-O- $\alpha$-di-rhamnopyranoside & P. densispica & [38] \\
\hline & lagotiside & P. kansuensis & [45] \\
\hline & luteolin & $\begin{array}{c}\text { P. cephalantha } \\
\text { P. kansuensis } \\
\text { P. longiflora var. tubiformis } \\
\text { P. rex } \\
\text { P. tricolor }\end{array}$ & {$[25,45,57,69,85]$} \\
\hline
\end{tabular}


Table 2. Cont.

\begin{tabular}{|c|c|c|c|}
\hline \multirow[t]{11}{*}{ Phytochemical Class } & Phytochemical Compound & Pedicularis spp. & References \\
\hline & luteolin-5-O-glucoside & P. longiflora var. tubiformis & [57-59] \\
\hline & luteolin-7-O-glucoside & $\begin{array}{c}\text { P. chamissonis } \\
\text { P. chinensis } \\
\text { P. kansuensis } \\
\text { P. longiflora var. tubiformis } \\
\text { P. rex } \\
\text { P. sibthorpii } \\
\text { P. sylvatica }\end{array}$ & {$[11,26,28,45,57,69,74]$} \\
\hline & luteolin-7-O-glucuronide & $\begin{array}{c}\text { P. chamissonis } \\
\text { P. longiflora var. tubiformis }\end{array}$ & {$[26,57]$} \\
\hline & morelosin & P. longiflora var. tubiformis & [57-59] \\
\hline & myricetin-3'-methyl ester 7-O-glucopyranoside & P. tricolor & [85] \\
\hline & orientin & P. longiflora var. tubiformis & [57-59] \\
\hline & quercetin-7-O-galactoside & P. tricolor & [85] \\
\hline & scutellarein-7-O-glucoside & P. densispica & [38] \\
\hline & tricin & P. longiflora var. tubiformis & [57] \\
\hline & tricin-7-O-glucuronide & $\begin{array}{c}\text { P. kansuensis } \\
\text { P. longiflora var. tubiformis }\end{array}$ & {$[45,57]$} \\
\hline & $\begin{array}{c}\text { 1,3,5,6-tetrahydro-1-methoxyl-7 } \\
\text {-methyl-cyclopenta[c]pyran-4-carboxaldehyde }\end{array}$ & P. uliginosa & [86] \\
\hline & 3ß-butoxy-3,4-dihydro-aucubin & P. chinensis & [27] \\
\hline & 4-epi-alyxialactone & P. uliginosa & [86] \\
\hline & (4R)-4-hydroxymethyl-boschnialactone & P. uliginosa & [86] \\
\hline & 5-deoxy-puchelloside I & P. spicata & [75] \\
\hline & 6-O-acetyl-aucubin & P. condensata & [29] \\
\hline & 6-O-methyl-aucubin & $\begin{array}{l}\text { P. artselaeri } \\
\text { P. chinensis }\end{array}$ & {$[20,28]$} \\
\hline & 6-O-butyl-aucubin & P. chinensis & [27] \\
\hline & 6-O-butyl-epi-aucubin & P. chinensis & [27] \\
\hline
\end{tabular}


Table 2. Cont.

\begin{tabular}{|c|c|c|c|}
\hline Phytochemical Class & Phytochemical Compound & Pedicularis spp. & References \\
\hline & 6-O-ethyl-aucubin & P. rex & [69] \\
\hline & 6-O-ethyl-epi-aucubin & P. rex & [69] \\
\hline & 6-O-methyl-epi-aucubin & P. artselaeri & [20] \\
\hline & 6-deoxy-catalpol & P. procera & [22] \\
\hline & 7-O-acetyl-gardoside methyl ester & P. dolichocymba & [41] \\
\hline & 7-deoxy-8-epi-loganic acid & $\begin{array}{c}\text { P. artselaeri } \\
\text { P. kansuensis } \\
\text { P. palustris } \\
\text { P. longiflora var. tubiformis } \\
\text { P. verticillata }\end{array}$ & {$[19,46,52,57,88]$} \\
\hline & 7-deoxy-gardoside & $\begin{array}{c}\text { P. artselaeri } \\
\text { P. cephalantha }\end{array}$ & {$[20,25]$} \\
\hline & 8-O-acetyl-harpagide & P. striata & [77] \\
\hline & 8-epi-loganic acid & $\begin{array}{c}\text { P. armata } \\
\text { P. artselaeri } \\
\text { P. kansuensis } \\
\text { P. kerneri } \\
\text { P. palustris } \\
\text { P. procera } \\
\text { P. rostratocapitata } \\
\text { P. verticillata }\end{array}$ & {$[18,19,22,46,51,52,70,88]$} \\
\hline & 8-epi-loganin & $\begin{array}{c}\text { P. artselaeri } \\
\text { P. condensata } \\
\text { P. densispica } \\
\text { P. lasiophrys } \\
\text { P. palustris } \\
\text { P. sylvatica } \\
\text { P. torta }\end{array}$ & {$[20,29,39,52,53,84]$} \\
\hline
\end{tabular}


Table 2. Cont.

\begin{tabular}{|c|c|c|c|}
\hline Phytochemical Class & Phytochemical Compound & Pedicularis spp. & References \\
\hline & $\begin{array}{c}\text { (rel-1R,4S,4aS,7R,7aR)-7-methyl-hexahydro-1, } \\
\text { 4-(epoxymethano)-cyclopenta[c]pyran-3(1H)-one }\end{array}$ & P. uliginosa & [86] \\
\hline & $\begin{array}{l}\text { (rel-4aS,7R,7aR)-1,4a,5,6,7,7a-hexahydro-7-hydroxyl- } \\
\text { 7-methyl-cyclopenta[c]pyran-4-carboxaldehyde }\end{array}$ & P. uliginosa & [86] \\
\hline & $\begin{array}{c}\text { rel-(6R,5R,9R)-(2-oxa-bicyclo- } \\
{[3,3,0] \text { oct-3-one-8-en-9,8-diyl)-dimethanol }}\end{array}$ & P. chinensis & [28] \\
\hline & alyxialactone & P. uliginosa & [86] \\
\hline & argyol & P. densispica & [37] \\
\hline & artselaenin I & P. artselaeri & [19] \\
\hline & artselaenin III & P. artselaeri & [19] \\
\hline & artselaenin A & $\begin{array}{l}\text { P. artselaeri } \\
\text { P. uliginosa }\end{array}$ & {$[19,86]$} \\
\hline & artselaenin B & $\begin{array}{l}\text { P. artselaeri } \\
\text { P. uliginosa }\end{array}$ & {$[20,86]$} \\
\hline & artselaenin C & P. artselaeri & [20] \\
\hline
\end{tabular}


Table 2. Cont.

\begin{tabular}{|c|c|c|c|}
\hline Phytochemical Class & Phytochemical Compound & Pedicularis spp. & References \\
\hline & aucubin & $\begin{array}{c}\text { P. armata } \\
\text { P. artselaeri } \\
\text { P. bracteosa } \\
\text { P. cephalantha } \\
\text { P. chinensis } \\
\text { P. condensata } \\
\text { P. crenulata } \\
\text { P. decora } \\
\text { P. groenlandica } \\
\text { P. kansuensis } \\
\text { P. kerneri } \\
\text { P. lapponica } \\
\text { P. longiflora var. tubiformis } \\
\text { P. muscicola } \\
\text { P. nordmanniana } \\
\text { P. palustris } \\
\text { P. procera } \\
\text { P. punctata } \\
\text { P. racemosa } \\
\text { P. rex } \\
\text { P. rostratocapitata } \\
\text { P. sibthorpii } \\
\text { P. verticillata }\end{array}$ & $\begin{array}{c}{[18,20,22,25,27,29,32-36} \\
46,51,52,57,62,63,67,69,70 \\
74,88]\end{array}$ \\
\hline & bartsioside & P. chinensis & [27] \\
\hline & boschnaloside & $\begin{array}{c}\text { P. alaschanica } \\
\text { P. longiflora var. tubiformis } \\
\text { P. kansuensis } \\
\text { P. palustris } \\
\text { P. plicata } \\
\text { P. resupinata } \\
\text { P. verticillata }\end{array}$ & {$[16,17,46,52,57,65,88]$} \\
\hline & boschnarol & P. uliginosa & [86] \\
\hline & caryoptoside & $\begin{array}{l}\text { P. artselaeri } \\
\text { P. muscicola } \\
\text { P. verticillata }\end{array}$ & {$[20,60,61,88]$} \\
\hline & densispicnin $\mathrm{A}$ & P. densispica & [37] \\
\hline
\end{tabular}


Table 2. Cont.

\begin{tabular}{|c|c|c|c|}
\hline Phytochemical Class & Phytochemical Compound & Pedicularis spp. & References \\
\hline & densispicnin B & $\begin{array}{l}\text { P. densispica } \\
\text { P. uliginosa } \\
\text { P. verticillata }\end{array}$ & {$[37,86,87]$} \\
\hline & densispicnin $C$ & P. densispica & [39] \\
\hline & densispicnin D & P. densispica & [39] \\
\hline & dihydro-catalpolgenin & $\begin{array}{c}\text { P. striata } \\
\text { P. striata subsp. aracnoidea }\end{array}$ & {$[78,81]$} \\
\hline & dolichocymboside A & P. dolichocymba & [40] \\
\hline & dolichocymboside B & P. dolichocymba & [40] \\
\hline & dolichocymboside C & P. dolichocymba & [40] \\
\hline & dolichocymboside D & P. dolichocymba & [40] \\
\hline & euphrasin & P. verticillata & [87] \\
\hline & euphroside & $\begin{array}{l}\text { P. alaschanica } \\
\text { P. armata } \\
\text { P. cephalantha } \\
\text { P. crenulata } \\
\text { P. groenlandica } \\
\text { P. kansuensis } \\
\text { P. kerneri } \\
\text { P. lapponica } \\
\text { P. muscicola } \\
\text { P. nordmanniana } \\
\text { P. palustris } \\
\text { P. racemosa } \\
\text { P. resupinata } \\
\text { P. rex } \\
\text { P. rostratocapitata } \\
\text { P. sylvatica } \\
\text { P. verticillata }\end{array}$ & $\begin{array}{c}{[16-18,22,25,46,51,52,62,} \\
63,69,70,88]\end{array}$ \\
\hline & gardoside & P. procera & [22] \\
\hline
\end{tabular}


Table 2. Cont.

\begin{tabular}{|c|c|c|c|}
\hline Phytochemical Class & Phytochemical Compound & Pedicularis spp. & References \\
\hline & gardoside methyl ester & $\begin{array}{l}\text { P. artselaeri } \\
\text { P. condensata } \\
\text { P. dolichocymba } \\
\text { P. kansuensis } \\
\text { P. muscicola } \\
\text { P. palustris } \\
\text { P. resupinata } \\
\text { P. spicata } \\
\text { P. torta }\end{array}$ & {$[16,20,29,41,52,62,75,84]$} \\
\hline & geniposidic acid & $\begin{array}{l}\text { P. alaschanica } \\
\text { P. armata } \\
\text { P. kansuensis } \\
\text { P. longiflora } \\
\text { P. muscicola } \\
\text { P. nordmanniana } \\
\text { P. resupinata } \\
\text { P. verticillata }\end{array}$ & {$[16-18,46,48,55,62,63,88]$} \\
\hline & iridolactone & $\begin{array}{c}\text { P. chinensis } \\
\text { P. nordmanniana }\end{array}$ & {$[27,63]$} \\
\hline & ixoroside & $\begin{array}{l}\text { P. alaschanica } \\
\text { P. artselaeri } \\
\text { P. kansuensis } \\
\text { P. palustris }\end{array}$ & {$[16,17,20,46,52]$} \\
\hline & kansuenin & P. kansuensis & [46] \\
\hline & kansuenin B & $\begin{array}{l}\text { P. kansuensis } \\
\text { P. verticillata }\end{array}$ & {$[45,87]$} \\
\hline & kansuenoside & P. kansuensis & {$[46]$} \\
\hline & lamalbid & P. decora & [32-36] \\
\hline & ligustroside & P. verticillata & [70] \\
\hline
\end{tabular}


Table 2. Cont.

\begin{tabular}{|c|c|c|c|}
\hline Phytochemical Class & Phytochemical Compound & Pedicularis spp. & References \\
\hline & loganic acid & $\begin{array}{l}\text { P. longiflora } \\
\text { P. torta }\end{array}$ & {$[55,84]$} \\
\hline & longifloroside & P. longiflora & [55] \\
\hline & monomelittoside & $\begin{array}{c}\text { P. kerneri } \\
\text { P. rostratocapitata } \\
\text { P. verticillata }\end{array}$ & {$[51,70]$} \\
\hline & mussaenin $\mathrm{A}$ & P. densispica & [37] \\
\hline & mussaenoside & $\begin{array}{c}\text { P. armata } \\
\text { P. artselaeri } \\
\text { P. bracteosa } \\
\text { P. cephalantha } \\
\text { P. condensata } \\
\text { P. densispica } \\
\text { P. groenlandica } \\
\text { P. kansuensis } \\
\text { P. lapponica } \\
\text { P. longiflora } \\
\text { P. muscicola } \\
\text { P. nordmanniana } \\
\text { P. palustris } \\
\text { P. procera } \\
\text { P. rex } \\
\text { P. semitorta }\end{array}$ & $\begin{array}{c}{[18,19,22,25,29,37,46,52} \\
55,62,63,69,73]\end{array}$ \\
\hline & mussaenosidic acid & $\begin{array}{c}\text { P. alaschanica } \\
\text { P. cephalantha } \\
\text { P. kerneri } \\
\text { P. longiflora var. tubiformis } \\
\text { P. muscicola } \\
\text { P. rostratocapitata } \\
\text { P. verticillata }\end{array}$ & {$[16,17,25,51,57,62,70]$} \\
\hline & ningpogoside B & P. decora & [32-36] \\
\hline
\end{tabular}


Table 2. Cont.

\begin{tabular}{|c|c|c|c|}
\hline Phytochemical Class & Phytochemical Compound & Pedicularis spp. & References \\
\hline & pedicularioside & $\begin{array}{l}\text { P. muscicola } \\
\text { P. palustris }\end{array}$ & {$[52,62]$} \\
\hline & pedicularioside $\mathrm{F}$ & P. lasiophrys & {$[53]$} \\
\hline & pedicularislactone & P. chinensis & [27] \\
\hline & pedicularislactone glucoside & $\begin{array}{l}\text { P. chinensis } \\
\text { P. decora }\end{array}$ & {$[28,32-36]$} \\
\hline & pedicutricoside $\mathrm{A}$ & P. tricolor & [85] \\
\hline & pediverticilatasin $\mathrm{A}$ & P. verticillata & [87] \\
\hline & pediverticilatasin $\mathrm{B}$ & P. verticillata & [87] \\
\hline & pediverticilatasin $C$ & P. verticillata & [87] \\
\hline & penstemonoside & $\begin{array}{l}\text { P. muscicola } \\
\text { P. palustris }\end{array}$ & {$[52,62]$} \\
\hline & plantarenaloside & $\begin{array}{c}\text { P. artselaeri } \\
\text { P. cephalantha } \\
\text { P. crenulata } \\
\text { P. decora } \\
\text { P. kerneri } \\
\text { P. palustris } \\
\text { P. resupinata } \\
\text { P. rex } \\
\text { P. rostratocapitata } \\
\text { P. sylvatica } \\
\text { P. verticillata }\end{array}$ & $\begin{array}{c}{[16,19,22,25,37,51,52,69} \\
88]\end{array}$ \\
\hline & phloyoside II & P. muscicola & {$[60,61]$} \\
\hline & plicatoside A & P. plicata & [65] \\
\hline & plicatoside B & P. plicata & [65] \\
\hline & proceroside & P. procera & [66] \\
\hline & scyphiphin A1 & P. verticillata & [87] \\
\hline & scyphiphin A2 & P. verticillata & [87] \\
\hline & sesamoside & P. muscicola & {$[60,61]$} \\
\hline
\end{tabular}


Table 2. Cont.

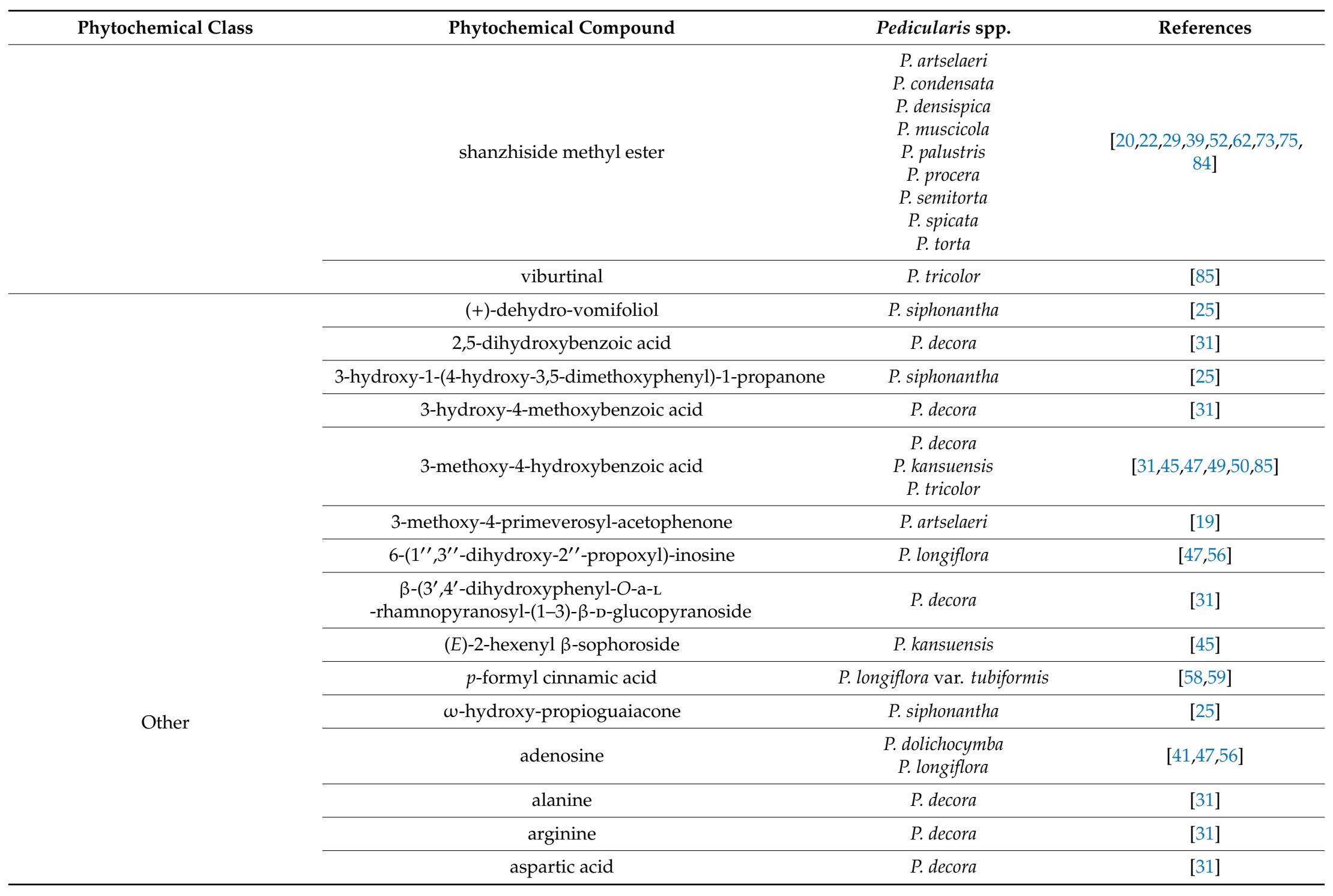


Table 2. Cont.

\begin{tabular}{|c|c|c|c|}
\hline Phytochemical Class & Phytochemical Compound & Pedicularis spp. & References \\
\hline & cinnamic acid & P. longiflora var. tubiformis & {$[58,59]$} \\
\hline & cysteine & P. decora & [31] \\
\hline & D-mannitol & $\begin{array}{c}\text { P. decora } \\
\text { P. kerneri } \\
\text { P. muscicola } \\
\text { P. sibthorpii }\end{array}$ & {$[31,51,62,74]$} \\
\hline & dearabinosyl-pneumonanthoside & P. densispica & [38] \\
\hline & eremophila-10,11-dien-7a,13-diol & P. striata subsp. aracnoidea & {$[80,81]$} \\
\hline & glutamic acid & P. decora & [31] \\
\hline & glycine & P. decora & [31] \\
\hline & isoleucine & P. decora & [31] \\
\hline & leucine & P. decora & [31] \\
\hline & maltol- $\beta$-D-glucoside & P. densispica & [38] \\
\hline & methionine & P. decora & [31] \\
\hline & muconic acid & P. longiflora var. tubiformis & {$[58,59]$} \\
\hline & pedicurexoside & P. rex & [69] \\
\hline & phenylalanine & P. decora & [31] \\
\hline & proline & P. decora & [31] \\
\hline & salicylic acid & P. decora & [31] \\
\hline & serine & P. decora & [31] \\
\hline & threonine & P. decora & [31] \\
\hline & tyrosine & P. decora & [31] \\
\hline & uridine & P. dolichocymba & [41] \\
\hline & valine & P. decora & [31] \\
\hline & vomifoliol & P. siphonantha & [25] \\
\hline
\end{tabular}


The structures of the majority of the identified compounds in Pedicularis species are reported in the figures below (Figures 3-21).<smiles>CC1(C)CCCC1(C)CO</smiles>

hexatriacontanol<smiles>CCC(C)(C)C(C)(C)C</smiles>

hentriacontane<smiles>CC(C)(C)CO</smiles>

nonatriacontanol<smiles>CC(C)(C)CC(=O)O</smiles>

arachidic acid<smiles>COc1cc2ccc(=O)oc2cc1O</smiles>

scopoletin<smiles>O=c1c2ccccc2oc2cccc(O)c12</smiles>

1-hydroxy-xanthone

Figure 3. Fatty acids, alkanes, alkyl alcohols, coumarins, and xanthones identified in Pedicularis species.

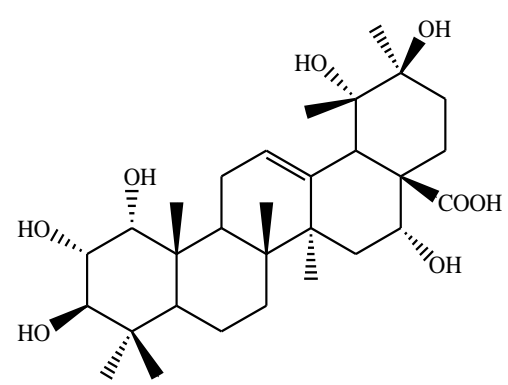

1,2,3,16,19,20-hexahydroxyolean-12-en-28-oic acid<smiles>C[C@H]1CC[C@]2(C(=O)O)CC[C@]3(C)C(=CCC4[C@@]5(C)CC(=O)[C@@H](O)C(C)(C)[C@H]5CC[C@]43C)C2[C@@]1(C)O</smiles>

3ß,19 $\alpha$-dihydroxy-12-ursen-28-oic acid

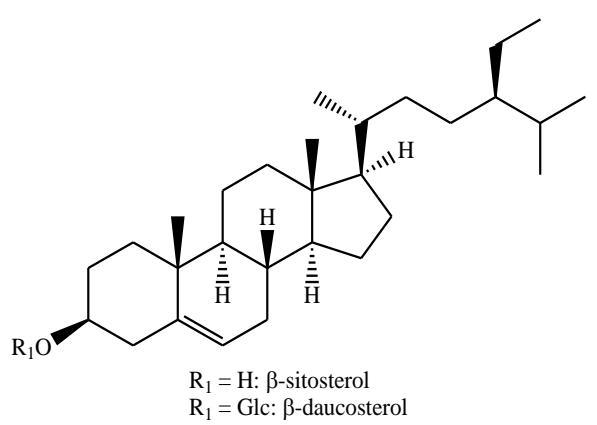<smiles>CC(=O)[C@]1(O)CC[C@]2(O)[C@]3(O)CC=C4C[C@@H](O)CC[C@]4(C)[C@]3(O)C[C@H](OC(=O)/C=C/c3ccccc3)[C@]12O</smiles>

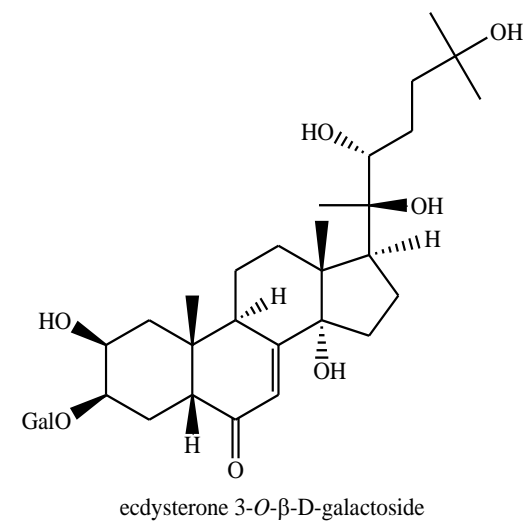

Figure 4. Terpenoids identified in Pedicularis species. 
<smiles>C1CCN2C[C@@H]3[C@H]4C[C@@H]4CN3C[C@@H]2C1</smiles>
lupanine<smiles>C[C@@H]1CCc2c(C(=O)O)cncc21</smiles>

plantagonine<smiles>CC1C(CO)=Cc2cccnc21</smiles><smiles>O=C1CCC[C@@H]2[C@@H]1CN1C[C@@H]3CCCCN3C[C@@H]21</smiles><smiles>C[C@@H]1CCc2c(C(=O)O)cncc21</smiles>

plantagonin<smiles>CC[n+]1cc(C=O)c2c(c1)C(C)CC2</smiles>

indicainine

Figure 5. Alkaloids identified in Pedicularis species-part 1.<smiles>C=CCCN1C[C@H]2C[C@H]3CN4C(=O)CCC[C@H]4C[C@H]3[C@H]2C1</smiles>

tetrahydrorhombifoline<smiles>CN1CC2CC(C1)c1cccc(=O)n1C2</smiles>

$\mathrm{N}$-methyl-cytisine pediculine

indicaine<smiles>C[C@@H]1CCc2c(C=O)cncc21</smiles><smiles>CC(C)[C@](O)(C(=O)OCC1=CCN2CC[C@H](O)[C@H]12)[C@H](C)O</smiles>

indicine<smiles>O=c1cccc2n1CC1C3CCCCN3CC1C21CC1</smiles>

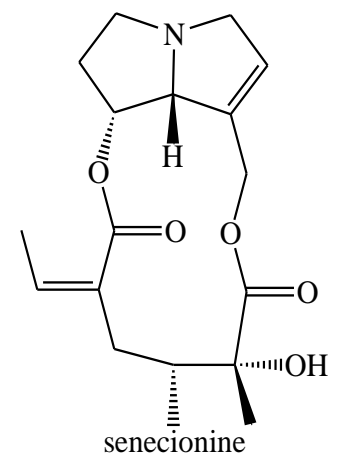

pediculidine

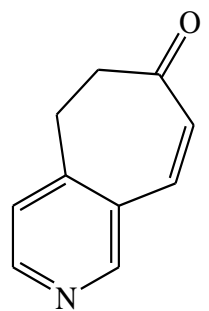

Figure 6. Alkaloids identified in Pedicularis species-part 2. 
<smiles>[R6]C[C@@H]1c2cc(CCCO)cc(OC)c2OC1c1ccc([R60])c(OC)c1</smiles>

$\mathrm{R}_{1}=\mathrm{Glc}, \mathrm{R}_{2}=\mathrm{H}$ : dihydro-dehydro-diconiferyl alcohol-4- $O-\beta$-D-glucoside $\mathrm{R}_{1}=\mathrm{H}, \mathrm{R}_{2}=$ Glc: dihydro-dehydro-diconiferyl alcohol-9-O- $\beta$-D-glucoside $\mathrm{R}_{1}=\mathrm{Rha}, \mathrm{R}_{2}=\mathrm{H}$ : dihydro-dehydro-diconiferyl alcohol-4-O- $\alpha$-L-rhamnoside<smiles>COC[C@H]1[C@@H](c2ccc(O)c(OC)c2)c2cc(O)c(OC)cc2C[C@H]1CO</smiles>

(+)-isolariciresinol 3a-O- $\beta$-D-glucopyranoside<smiles>COc1cc([C@H]2c3cc(O)c(OC)cc3C[C@@H](CO)[C@H]2COC(C)=O)ccc1O</smiles>
densispicoside<smiles>COc1cc([C@@H](O)[C@H](CO)Oc2c(OC)cc(/C=C/C=O)cc2OC)ccc1O[C@@H]1O[C@H](CO)[C@@H](O)[C@H](O)[C@H]1O</smiles><smiles>COc1ccc([C@H]2Oc3cc(/C=C/CO)cc(OC)c3O[C@H]2CO)cc1OC</smiles>

7S: verticillatoside A
7R: verticillatoside B

Figure 7. Lignans and neo-lignans identified in Pedicularis species-part 1.<smiles>COc1cc(C(O)C(CO)Oc2ccc(/C=C\COC3CCCCC3)cc2O)ccc1O</smiles>
erythro: semitortoside A<smiles>COc1ccc([C@@H]2Cc3c(OC)cc(/C=C/COCl)cc3C2CO)cc1OC</smiles>

longifloroside B<smiles>[R6]Oc1ccc([C@H]2OC[C@@H](Cc3ccc(OC)c(OC)c3)[C@@H]2CO)cc1OC</smiles>

$\mathrm{R}_{1}=\mathrm{Glc}, \mathrm{R}_{2}=\mathrm{H}$ : lariciresinol-4-O- $\beta$-D-glucoside $\mathrm{R}_{1}=\mathrm{H}, \mathrm{R}_{2}=$ Glc: lariciresinol-4'-O- $\beta$-D-glucoside

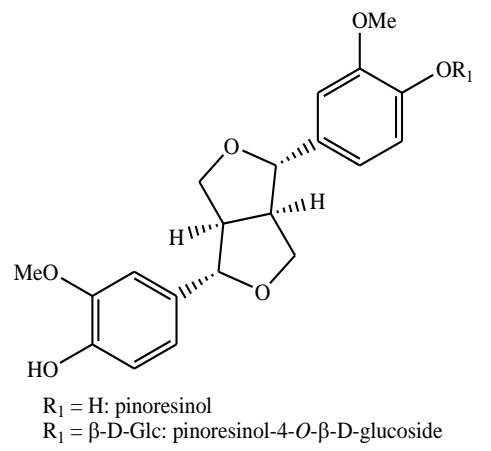

Figure 8. Lignans and neo-lignans identified in Pedicularis species-part 2. 
<smiles>[R3]C1c2cc(/C=C/CO)cc(OC)c2OC1c1ccc(OC)c(OC)c1</smiles>

$\mathrm{R}_{1}=(R)-\mathrm{CH}_{2} \mathrm{OH}: 7(R)$-dehydro-diconiferyl alcohol-4- $O$ - $\beta$-glucoside $\mathrm{R}_{1}=(S)-\mathrm{CH}_{2} \mathrm{OH}$ : 7(S)-dehydro-diconiferyl alcohol-4-O- $\beta$-glucoside

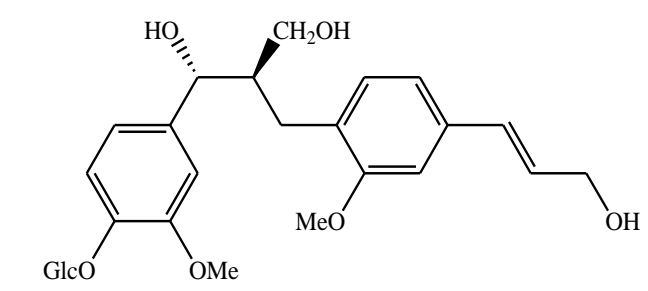<smiles>[R20]c1c(OC)cc([C@@H]2OC[C@H]3[C@@H](c4cc(OC)c(OC([R])([R])O)c(OC)c4)OC[C@H]32)cc1OC</smiles>

$R_{1}=\beta-D-G l c, R_{2}=H$ : syringaresinol-4- $O-\beta-D$-glucoside $\mathrm{R}_{1}=\mathrm{H}, \mathrm{R}_{2}=\beta$-D-Glc: syringaresinol-4"-O- $\beta$-D-glucoside

alaschanioside A<smiles>COc1ccc([C@@H]2Cc3c(OC)cc(/C=C/CO)cc3[C@H]2CO)cc1OC</smiles><smiles>[R]c1cc(/C=C/CO)cc(OC)c1OC(CO)C(O)c1ccc(OC2CCCCC2)c(OC)c1</smiles>

$\mathrm{R}_{1}=\mathrm{H}$ : citrusin A

$\mathrm{R}_{1}=\mathrm{OMe}:$ citrusin $\mathrm{B}$

alaschanioside $\mathrm{C}$

Figure 9. Lignans and neo-lignans identified in Pedicularis species-part 3.

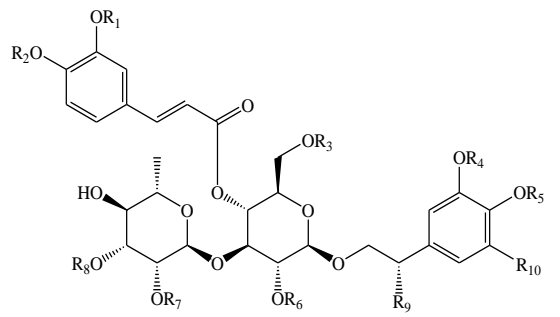

$R_{1}=R_{2}=R_{3}=R_{4}=R_{5}=R_{6}=R_{7}=R_{8}=R_{9}=R_{10}=H_{1} \cdot$ vethscoside $R_{1}=R_{2}=R_{4}=R_{5}=$ Me, $R_{3}=R_{6}=R_{7}=R_{8}=R_{9}=R_{10}=H$ : permethyl-verbascoside $\mathrm{R}_{1}=\mathrm{Me}, \mathrm{R}_{2}=\mathrm{R}_{3}=\mathrm{R}_{4}=\mathrm{R}_{5}=\mathrm{R}_{6}=\mathrm{R}_{7}=\mathrm{R}_{8}=\mathrm{R}_{9}=\mathrm{R}_{10}=\mathrm{H}$ : leucosceptoside $\mathrm{A}$ $\mathrm{R}_{1}=\mathrm{R}_{2}=\mathrm{R}_{4}=\mathrm{R}_{5}=\mathrm{R}_{6}=\mathrm{R}_{7}=\mathrm{R}_{8}=\mathrm{R}_{9}=\mathrm{R}_{10}=H, \mathrm{R}_{3}=\beta$-D-Glc:echinacoside $R_{1}=R_{5}=M e, R_{2}=R_{3}=R_{4}=R_{6}=R_{7}=R_{8}=R_{9}=R_{10}=H$ : martynoside

$\mathrm{R}_{1}=\mathrm{R}_{2}=\mathrm{R}_{4}=\mathrm{R}_{5}=\mathrm{R}_{6}=\mathrm{R}_{7}=\mathrm{R}_{8}=\mathrm{R}_{9}=\mathrm{R}_{10}=\mathrm{H}, \mathrm{R}_{3}=\beta$-D-Api: forsythoside $\mathrm{B}$

$R_{1}=R_{5}=M e, R_{2}=R_{3}=R_{4}=R_{6}=R_{8}=R_{9}=R_{0}=H, R_{7}=A c .2^{2}$-acetyl-martynoside $\mathrm{R}_{1}=\mathrm{R}_{2}=\mathrm{R}_{3}=\mathrm{R}_{4}=\mathrm{R}_{6}=\mathrm{R}_{7}=\mathrm{R}_{8}=\mathrm{R}_{9}=\mathrm{R}_{10}=\mathrm{H}, \mathrm{R}_{5}=\mathrm{Re}$. Jionoside $\mathrm{D}$

$R_{1}=R_{5}=M e, R_{2}=R_{3}=R_{4}=R_{6}=R_{9}=R_{10}=H, R_{7}=R_{8}=$ Ac. clerodenoside A

$R_{1}=R_{2}=R_{4}=R_{5}=R_{6}=R_{7}=R_{8}=R_{10}=H, R_{3}=\beta$-D-Glc, $R_{9}=O H$ : cistanbuloside C1

$R_{1}=R_{4}=M e, R_{2}=R_{3}=R_{5}=R_{6}=R_{7}=R_{8}=R_{9}=R_{10}=H$ : cistanoside $D$

$R_{1}=R_{5}=M e, R_{2}=R_{4}=R_{6}=R_{7}=R_{8}=R_{9}=R_{10}=H, R_{3}=\beta$-D-Gal: jionoside $B 1$

$\mathrm{R}_{1}=\mathrm{R}_{2}=\mathrm{R}_{3}=\mathrm{R}_{4}=\mathrm{R}_{5}=\mathrm{R}_{6}=\mathrm{R}_{7}=\mathrm{R}_{8}=\mathrm{R}_{9}=\mathrm{H}, \mathrm{R}_{10}=\mathrm{OH}$ : 1 -(2,3,4-trihydroxyphenyl $)$
ethyl-3-O-rhamnose-4-[(2E)-3-(3,4-dihydroxyphenyl)-2-propenoate)-glucopyranoside
$\mathrm{HO}$
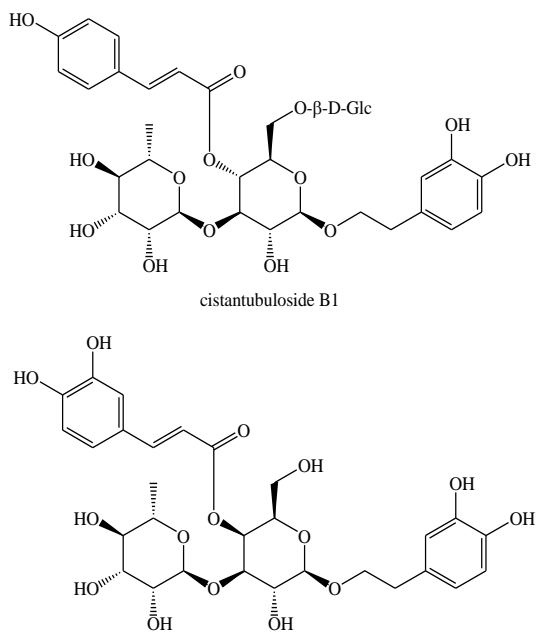

pedicularioside $\mathrm{G}$

Figure 10. Phenylethanoid glycosides identified in Pedicularis species-part 1. 


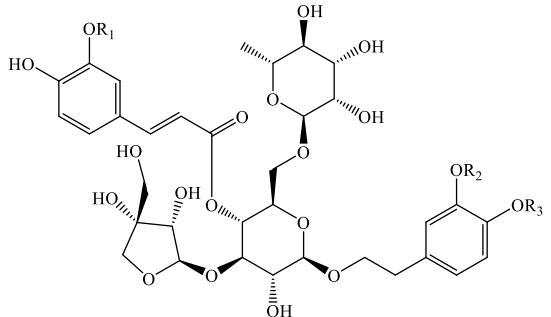

$\mathrm{R}_{1}=\mathrm{R}_{2}=\mathrm{R}_{3}=\mathrm{H}$ : pedicularioside $\mathrm{A}$ $\mathrm{R}_{1}=\mathrm{R}_{2}=\mathrm{Me}, \mathrm{R}_{3}=\mathrm{H}$ : pedicularioside $\mathrm{H}$ $\mathrm{R}_{1}=\mathrm{R}_{3}=\mathrm{Me}, \mathrm{R}_{2}=\mathrm{H}$ : pedicularioside $\mathrm{N}$ $R_{1}=M e, R_{2}=R_{3}=H$ : pedicularioside $M$

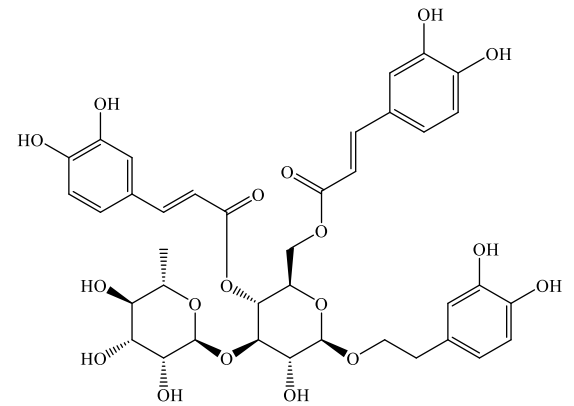

1-(2,3,4-trihydroxyphenyl) ethyl-3-O-rhamnose-4-[(2E)-3-(3,4-dihydroxyphenyl)-2propenoate $]-6-[(2 E)$-3-(3,4-dihydroxyphenyl)-2-propenoate]-glucopyranoside

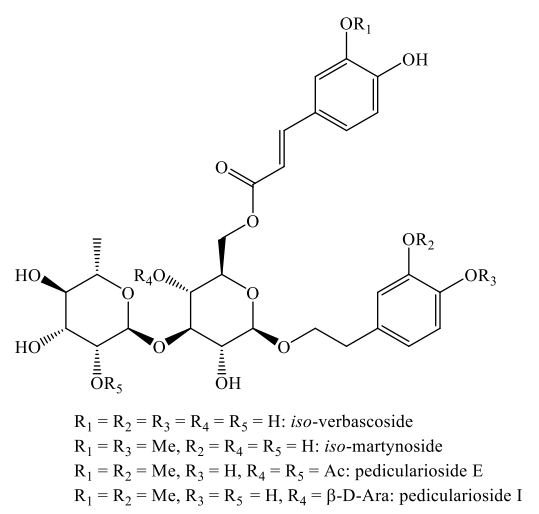

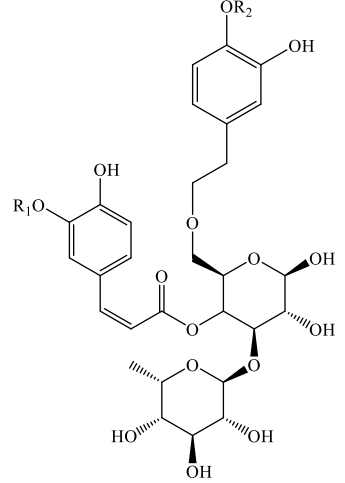

$\mathrm{R}_{1}=\mathrm{R}_{2}=\mathrm{H}$ : cis-iso-verbascoside $\mathrm{R}_{1}=\mathrm{R}_{2}=$ Me: cis-iso-martynoside

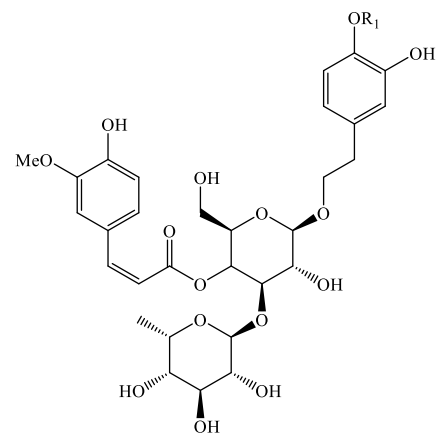

$\mathrm{R}_{1}=\mathrm{H}:$ cis-leucosceptoside $\Lambda$ $\mathrm{R}_{1}=$ Me: cis-martynoside

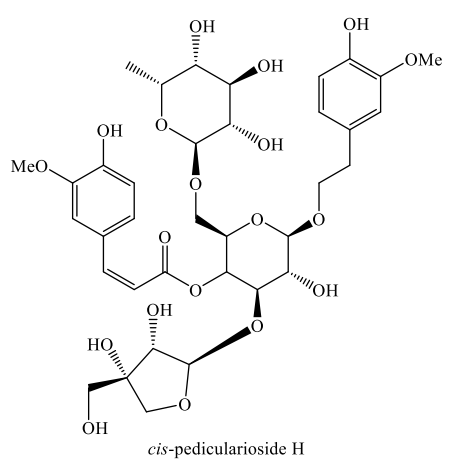

Figure 11. Phenylethanoid glycosides identified in Pedicularis species-part 2. 


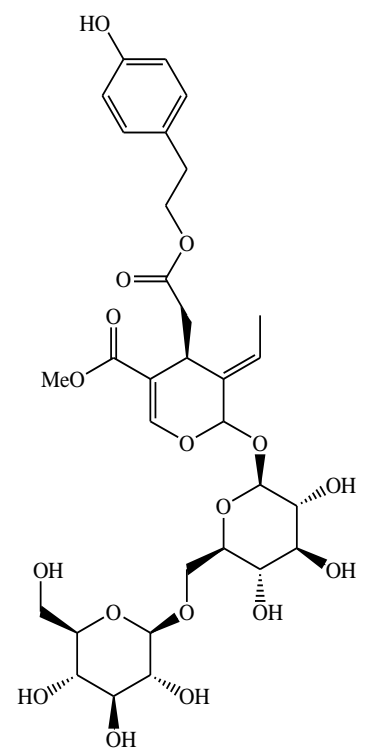

excelside B
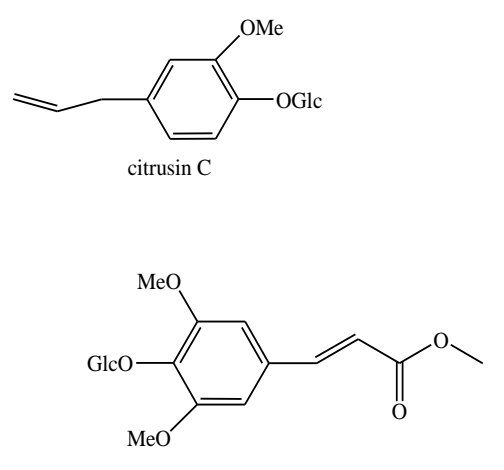

4-O- $\beta$-D-glucopyranosyl-sinapic acid methyl ester

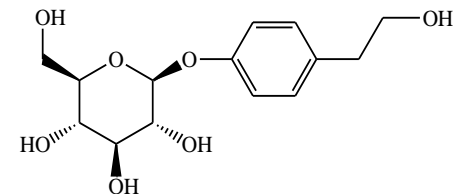

2-(p-hydroxyphenyl)-ethanol-1-O- $\beta$-D-glucopyranoside

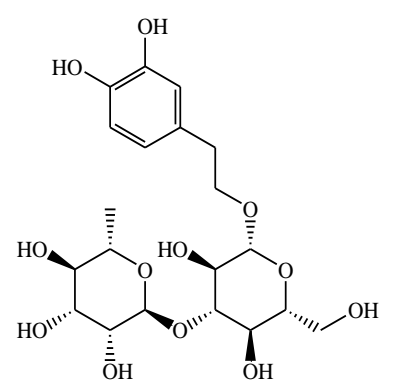

decaffeoyl-verbascoside

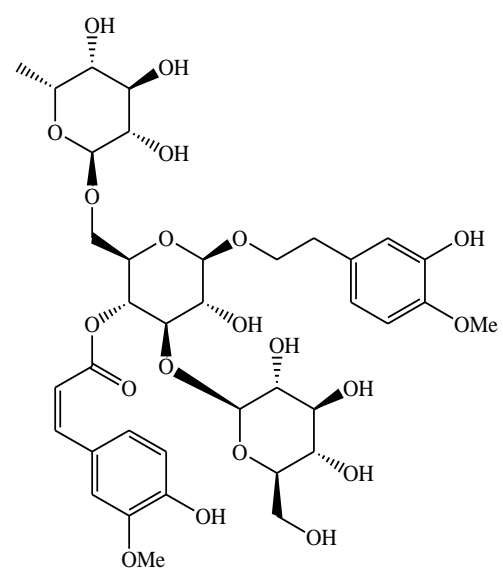

artselaeroside B

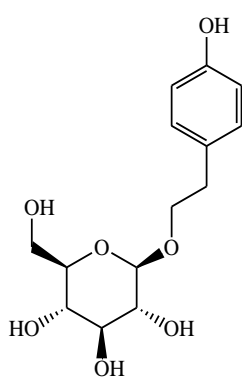

salidroside
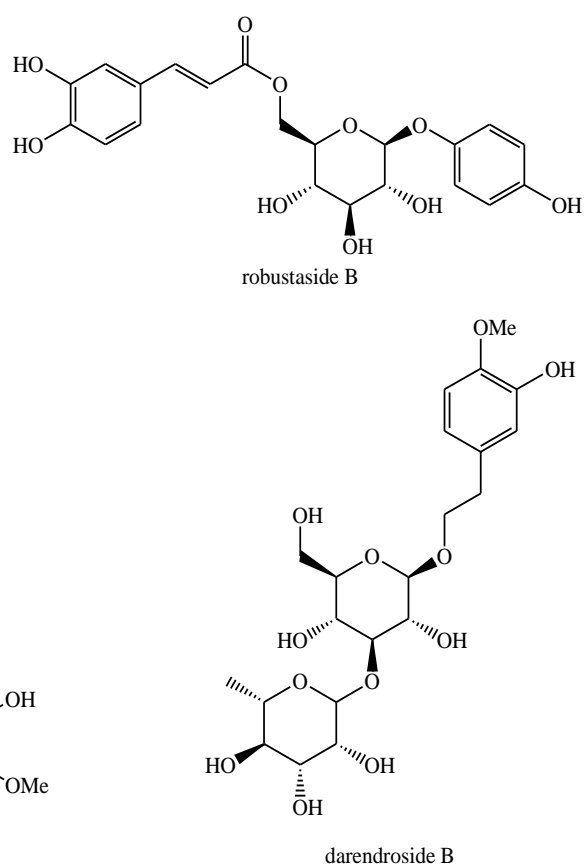

darendroside B<smiles>OC[C@H]1O[C@H](OCCc2ccccc2)[C@@H](O)[C@H](O[C@@H]2OC[C@@H](O)[C@H](O)[C@H]2O)[C@H]1O</smiles>

artselaeroside A

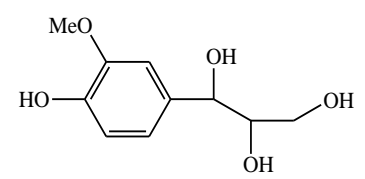

3-(4-hydroxy-3-methoxyphenyl)-1,2,3-propantriol

Figure 12. Phenylethanoid glycosides identified in Pedicularis species-part 3. 
<smiles>[R]c1cc(-c2cc(=O)c3c(O)cc(O)cc3o2)cc([R])c1[R]</smiles>

$\mathrm{R}_{1}=\mathrm{R}_{3}=\mathrm{H}, \mathrm{R}_{2}=\mathrm{OH}$ : apigenin

$\mathrm{R}_{1}=\mathrm{R}_{3}=\mathrm{H}, \mathrm{R}_{2}=$ OMe: acacetin

$\mathrm{R}_{1}=\mathrm{R}_{2}=\mathrm{OH}, \mathrm{R}_{3}=\mathrm{H}$ : luteolin

$\mathrm{R}_{1}=\mathrm{OMe}, \mathrm{R}_{2}=\mathrm{OH}, \mathrm{R}_{3}=\mathrm{H}$ : chrysoeriol

$\mathrm{R}_{1}=\mathrm{R}_{2}=\mathrm{OMe}, \mathrm{R}_{3}=\mathrm{H}$ : 4'-methyl-chrysoeriol

$\mathrm{R}_{1}=\mathrm{R}_{3}=\mathrm{OMe}, \mathrm{R}_{2}=\mathrm{H}$ : morelosin

$\mathrm{R}_{1}=\mathrm{R}_{3}=\mathrm{OMe}, \mathrm{R}_{2}=\mathrm{OH}$ : tricin<smiles>O=c1c(O)c(-c2ccc(O)cc2)oc2cc(O)cc(O)c12</smiles><smiles>[R3]c1cc(-c2oc3cc(O)cc(O)c3c(=O)c2[R])cc(Br)c1[R3]</smiles>

$\mathrm{R}_{1}=\mathrm{H}, \mathrm{R}_{2}=\mathrm{OH}, \mathrm{R}_{3}=\mathrm{R}_{4}=\mathrm{OMe}: 3,3$ '-di- $O$-methyl-quercetin<smiles>O=c1cc(-c2ccc(O)c(O)c2)oc2cc(O)cc(O[C@@H]3O[C@H](CO)[C@@H](O)[C@H](O)[C@H]3O)c12</smiles>

luteolin-5-O-glucoside<smiles>COc1ccc(-c2oc3cc(O[C@@H]4O[C@H](CO)[C@@H](O)[C@H](O)[C@H]4O)cc(O)c3c(=O)c2O)cc1OC</smiles><smiles>O=c1cc(-c2ccc(O)c(O)c2)oc2c([C@@H]3O[C@H](CO)[C@@H](O)[C@H](O)[C@H]3O)c(O)cc(O)c12</smiles>

Figure 13. Flavonoids identified in Pedicularis species-part 1. 
<smiles>[R]c1cc(-c2cc(=O)c3c(O)c([R])c(O[C@@H]4O[C@H]([R])[C@@H](C)[C@H](O)[C@H]4O)cc3o2)cc([R3])c1O</smiles>

$\mathrm{R}_{1}=\mathrm{R}_{2}=\mathrm{R}_{3}=\mathrm{H}, \mathrm{R}_{4}=\mathrm{CH}_{2} \mathrm{OH}$ :apigenin-7-O-glucoside

$\mathrm{R}_{1}=\mathrm{R}_{2}=\mathrm{R}_{3}=\mathrm{H}, \mathrm{R}_{4}=$ COOH:apigenin-7-O-glucuronide

$\mathrm{R}_{1}=\mathrm{OH}, \mathrm{R}_{2}=\mathrm{R}_{3}=\mathrm{H}, \mathrm{R}_{4}=\mathrm{CH}_{2} \mathrm{OH}$ : luteolin-7- $O$-glucoside

$\mathrm{R}_{1}=\mathrm{OH}, \mathrm{R}_{2}=\mathrm{R}_{3}=\mathrm{H}, \mathrm{R}_{4}=\mathrm{COOH}$ : luteolin-7-O-glucuronide

$\mathrm{R}_{1}=\mathrm{R}_{2}=\mathrm{H}, \mathrm{R}_{3}=\mathrm{OH}, \mathrm{R}_{4}=\mathrm{CH}_{2} \mathrm{OH}$ :scutellarein-7-O-glucoside

$\mathrm{R}_{1}=\mathrm{OMe}, \mathrm{R}_{2}=\mathrm{R}_{3}=\mathrm{H}, \mathrm{R}_{4}=\mathrm{CH}_{2} \mathrm{OH}$ :chrysoeriol-7-O-glucoside

$\mathrm{R}_{1}=\mathrm{OMe}, \mathrm{R}_{2}=\mathrm{R}_{3}=\mathrm{H}, \mathrm{R}_{4}=\mathrm{COOH}$ :chrysoeriol-7- $O$-glucuronide

$\mathrm{R}_{1}=\mathrm{R}_{2}=\mathrm{OMe}, \mathrm{R}_{3}=\mathrm{H}, \mathrm{R}_{4}=\mathrm{COOH}$ : tricin-7-O-glucuronide<smiles>[R3]c1cc(-c2oc3cc(O[C@@H]4O[C@H](CO)[C@@H](O)[C@H](O)[C@H]4O)cc(O)c3c(=O)c2[R])cc([R9])c1O</smiles><smiles>COc1cc(-c2cc(=O)c3c(O)cc(O[C@@H]4O[C@H](CO)[C@@H](O)[C@H](O)[C@H]4O)cc3o2)ccc1O</smiles>

5,4'-di-hydroxy-3'-methoxy-flavone-7-O-6"--n-butyryl- $\beta$-D-glucopyranoside<smiles>C/C(=C\C=C\O)c1oc2cc(O[C@@H]3O[C@H](C)[C@@H](O)[C@H](O)[C@H]3O)cc(O)c2c(=O)c1O[C@@H]1O[C@H](C)[C@@H](O)[C@H](O)[C@H]1O</smiles>

kaempferol-3,7-O- $\alpha$-di-rhamnopyranoside

$\mathrm{R}_{1}=\mathrm{R}_{2}=\mathrm{OMe}, \mathrm{R}_{3}=\mathrm{OH}: 3,5,4^{\prime}$-trihydroxy-3',5'-dimethoxy-flavone-7- $O$-glucoside $\mathrm{R}_{1}=\mathrm{OMe}, \mathrm{R}_{2}=\mathrm{R}_{3}=\mathrm{OH}: 3,5,4^{\prime}, 5^{\prime}$-tetrahydroxy-3'-methoxy-flavone-7-O-glucoside $\mathrm{R}_{1}=\mathrm{R}_{3}=\mathrm{OH}, \mathrm{R}_{2}=\mathrm{H}: 3,5,3^{\prime}, 4^{\prime}$-tetrahydroxy-flavone-7-O-glucoside $\mathrm{R}_{1}=\mathrm{R}_{3}=\mathrm{OH}, \mathrm{R}_{2}=\mathrm{H}: 3,5,3,4^{\prime}$-tetrahydroxy-flavone- $-O$-glucoside
$\mathrm{R}_{1}=\mathrm{R}_{2}=\mathrm{OH}, \mathrm{R}_{3}=\mathrm{OMe}$ : myricetin-3'-methyl ester 7-O-glucoside<smiles>CCOc1cc(O[C@@H]2O[C@H](CO)[C@@H](O)[C@H](O)[C@H]2O)cc2oc(-c3ccc(O)c(O)c3)c(O)c(=O)c12</smiles>

Figure 14. Flavonoids identified in Pedicularis species-part 2. 


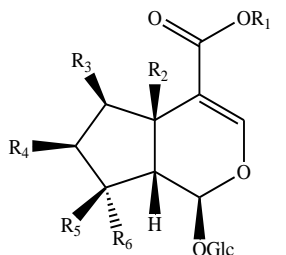

$\mathrm{R}_{1}=\mathrm{R}_{6}=\mathrm{Me}, \mathrm{R}_{2}=\mathrm{R}_{3}=\mathrm{H}, \mathrm{R}_{4}=\mathrm{R}_{5}=\mathrm{OH}$ : caryoptoside $\mathrm{R}_{1}=\mathrm{R}_{2}=\mathrm{R}_{3}=\mathrm{R}_{4}=\mathrm{H}, \mathrm{R}_{5}=\mathrm{OH}, \mathrm{R}_{6}=$ Me: mussaenosidic acid $\mathrm{R}_{1}=\mathrm{R}_{6}=\mathrm{Me}, \mathrm{R}_{2}=\mathrm{R}_{3}=\mathrm{R}_{4}=\mathrm{H}, \mathrm{R}_{5}=\mathrm{OH}$ : mussaenoside $\mathrm{R}_{1}=\mathrm{R}_{2}=\mathrm{R}_{3}=\mathrm{R}_{5}=\mathrm{H}, \mathrm{R}_{4}=\mathrm{OH}, \mathrm{R}_{6}=$ Me: 8-epi-loganic acid $\mathrm{R}_{1}=\mathrm{R}_{2}=\mathrm{R}_{3}=\mathrm{R}_{4}=\mathrm{R}_{5}=\mathrm{H}, \mathrm{R}_{6}=$ Me: 7-deoxy-8-epi-loganic acid $\mathrm{R}_{1}=\mathrm{R}_{6}=\mathrm{Me}, \mathrm{R}_{2}=\mathrm{R}_{3}=\mathrm{R}_{4}=\mathrm{R}_{5}=\mathrm{H}$ : 7-deoxy-8-epi-loganin $\mathrm{R}_{1}=\mathrm{R}_{6}=\mathrm{Me}, \mathrm{R}_{2}=\mathrm{R}_{3}=\mathrm{R}_{5}=\mathrm{H}, \mathrm{R}_{4}=\mathrm{OH}:$ 8-epi-loganin $\mathrm{R}_{1}=\mathrm{R}_{6}=\mathrm{Me}, \mathrm{R}_{2}=\mathrm{R}_{4}=\mathrm{H}, \mathrm{R}_{3}=\mathrm{R}_{5}=\mathrm{OH}$ : shanzhiside methyl ester $\mathrm{R}_{1}=\mathrm{R}_{6}=\mathrm{Me}, \mathrm{R}_{2}=\mathrm{H}, \mathrm{R}_{3}=\mathrm{R}_{4}=\mathrm{R}_{5}=\mathrm{OH}$ : lamalbid $\mathrm{R}_{1}=\mathrm{R}_{6}=\mathrm{Me}, \mathrm{R}_{2}=\mathrm{R}_{4}=\mathrm{R}_{5}=\mathrm{H}, \mathrm{R}_{3}=\mathrm{OH}$ : penstemonoside $\mathrm{R}_{1}=\mathrm{R}_{6}=\mathrm{Me}, \mathrm{R}_{2}=\mathrm{R}_{3}=\mathrm{OH}, \mathrm{R}_{4}=\mathrm{R}_{5}=\mathrm{H}$ : pedicularioside

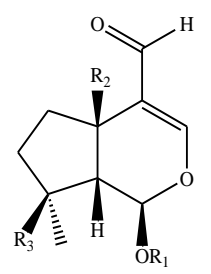

$\mathrm{R}_{1}=\mathrm{R}_{2}=\mathrm{R}_{3}=\mathrm{H}$ : boschnarol

$\mathrm{R}_{1}=\beta$-D-Glc, $\mathrm{R}_{2}=\mathrm{R}_{3}=\mathrm{H}$ : boschnalosid

$\mathrm{R}_{1}=\beta$-D-Glc, $\mathrm{R}_{2}=\mathrm{H}, \mathrm{R}_{3}=\mathrm{OH}$ : ixoroside

$R_{1}=\beta$-D-Glc, $R_{2}=R_{3}=$ OH. euphroside

$\mathrm{R}_{1}=\beta$-D-Glc, $\mathrm{R}_{2}=\mathrm{OH}, \mathrm{R}_{3}=\mathrm{H}$ : plantarenaloside
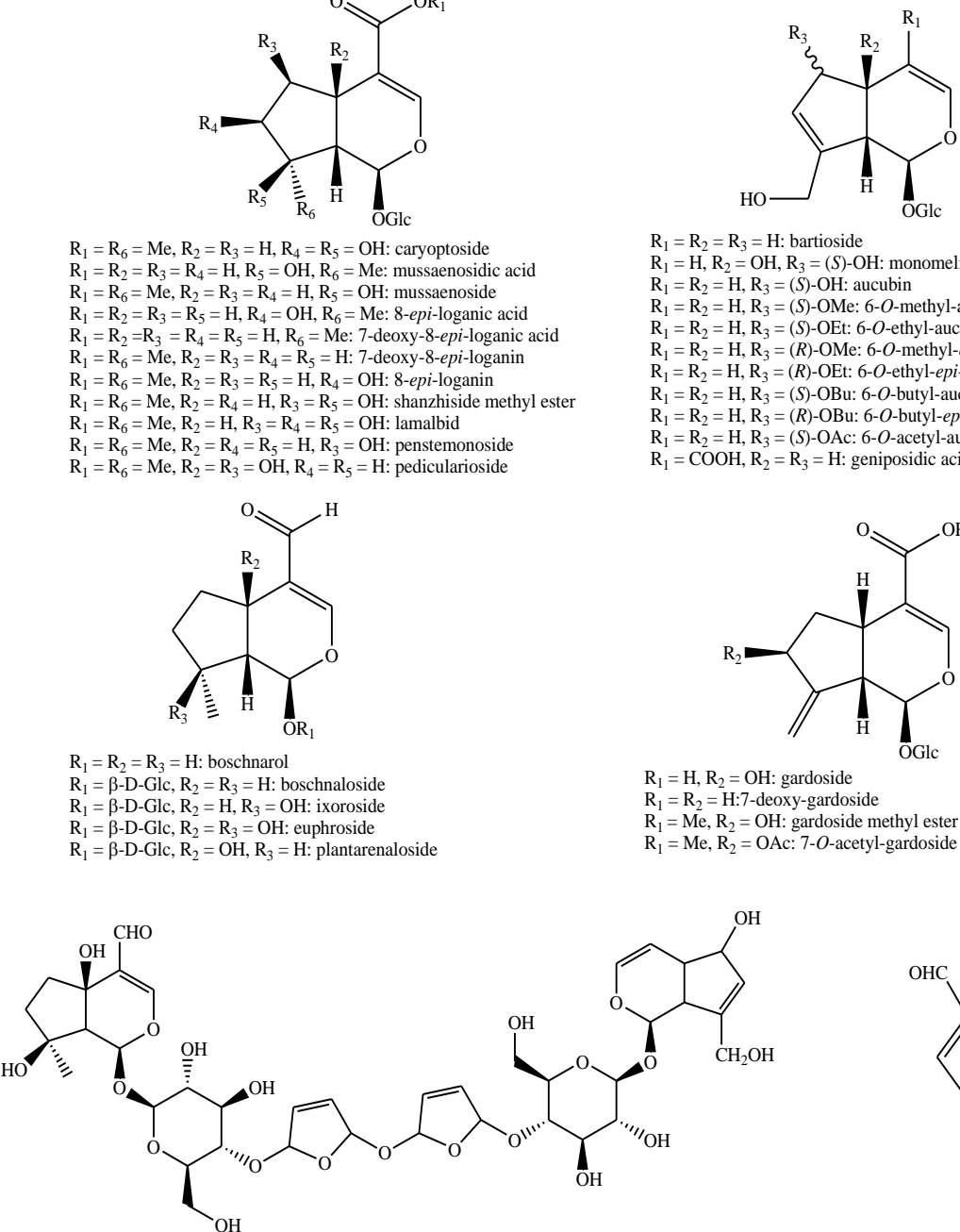

$\mathrm{R}_{1}=\mathrm{R}_{2}=\mathrm{R}_{3}=\mathrm{H}$ : bartioside

$\mathrm{R}_{1}=\mathrm{H}_{2}, \mathrm{R}_{2}=\mathrm{OH}, \mathrm{R}_{3}=(S)-\mathrm{OH}$ : monomelittoside $\mathrm{R}_{1}=\mathrm{R}_{2}=\mathrm{H}, \mathrm{R}_{3}=(S)-\mathrm{OH}$ : aucubin

$\mathrm{R}_{1}=\mathrm{R}_{2}=\mathrm{H}, \mathrm{R}_{3}=(S)$-OMe: 6 - $O$-methyl-aucubin $\mathrm{R}_{1}=\mathrm{R}_{2}=\mathrm{H}, \mathrm{R}_{3}=(S)$-OEt: 6-O-ethyl-aucubin $\mathrm{R}_{1}=\mathrm{R}_{2}=\mathrm{H}, \mathrm{R}_{3}=(R)$-OMe: 6-O-methyl-epi-aucubin $\mathrm{R}_{1}=\mathrm{R}_{2}=\mathrm{H}, \mathrm{R}_{3}=(R)$-OEt: 6-O-ethyl-epi-aucubi $\mathrm{R}_{1}=\mathrm{R}_{2}=\mathrm{H}, \mathrm{R}_{3}=(S)$-OBu: 6-O-butyl-aucubin $\mathrm{R}_{1}=\mathrm{R}_{2}=\mathrm{H}, \mathrm{R}_{3}=(R)$-OBu: 6-O-butyl-epi-aucubin $\mathrm{R}_{1}=\mathrm{R}_{2}=\mathrm{H}, \mathrm{R}_{3}=(\mathrm{S})-\mathrm{OAc}$ : 6-O-acetyl-aucubi
$\mathrm{R}_{1}=\mathrm{COOH}, \mathrm{R}_{2}=\mathrm{R}_{3}=\mathrm{H}$ : geniposidic acid

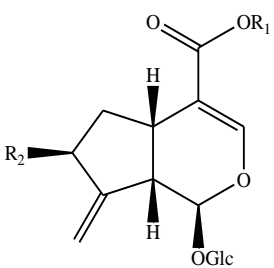

$\mathrm{R}_{1}=\mathrm{H}, \mathrm{R}_{2}=\mathrm{OH}$ : gardoside

$\mathrm{R}_{1}=\mathrm{R}_{2}=\mathrm{H}: 7$-deoxy-gardoside

$\mathrm{R}_{1}=\mathrm{Me}, \mathrm{R}_{2}=\mathrm{OH}$ : gardoside methyl ester

$\mathrm{R}_{1}=$ Me, $\mathrm{R}_{2}=$ OAc: 7 - $O$-acetyl-gardoside methyl ester

longifloroside

Figure 15. Iridoids identified in Pedicularis species-part 1.<smiles>CO[C@@H]1OC=C(CO)C2=CC[C@@H](C)[C@@H]21</smiles>

kansuenoside

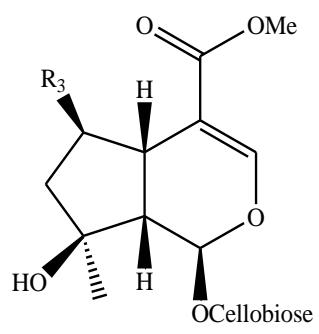

pedicularioside $\mathrm{F}$

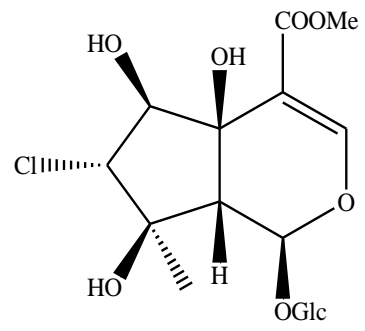

phloyoside II

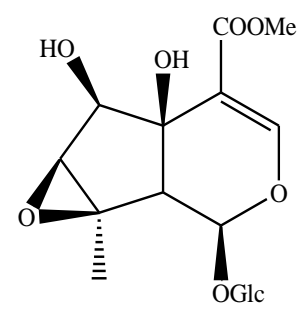

sesamoside

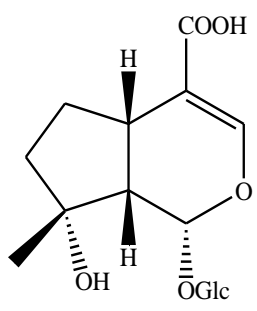

pedicutroside $\mathrm{A}$<smiles>CO[C@H]1OC=C[C@H]2CC(=O)[C@@H](CO)[C@@H]12</smiles>

proceroside

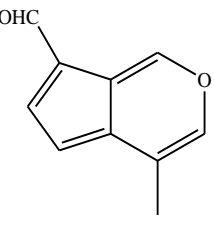

viburtinal

Figure 16. Iridoids identified in Pedicularis species-part 2. 


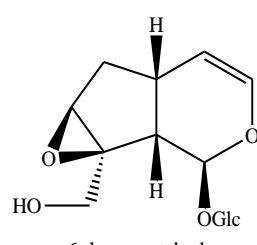

6-deoxy-catalpol

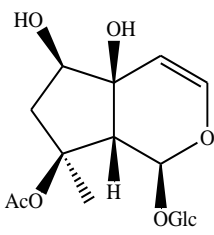

8-O-acetyl-harpagide

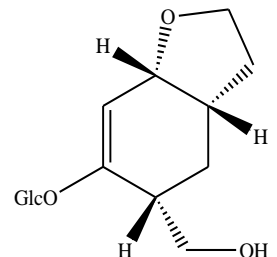

ningpogoside $\mathrm{B}$

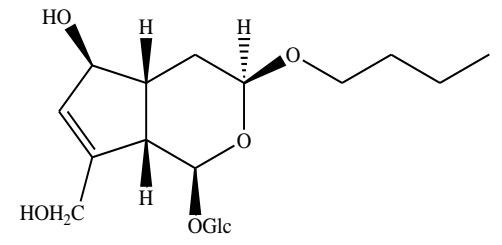

3ß-butoxy-3,4-dihydro-aucubin

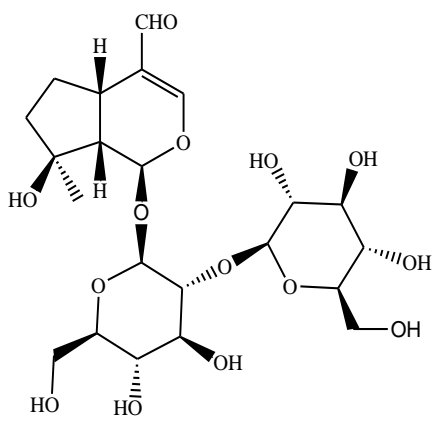

plicatoside A

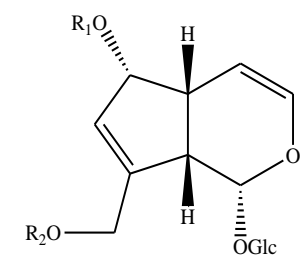

$\mathrm{R}_{1}=\mathrm{R}_{2}=$ Ac: dolichocymboside $\mathrm{A}$ $\mathrm{R}_{1}=\mathrm{H}_{2} \mathrm{R}_{2}=$ Ac: dolichocymboside $\mathrm{B}$ $\mathrm{R}_{1}=\mathrm{Ac}, \mathrm{R}_{2}=\mathrm{H}$ : dolichocymboside $\mathrm{C}$ $\mathrm{R}_{1}=\mathrm{R}_{2}=\mathrm{H}$ : dolichocymboside $\mathrm{D}$

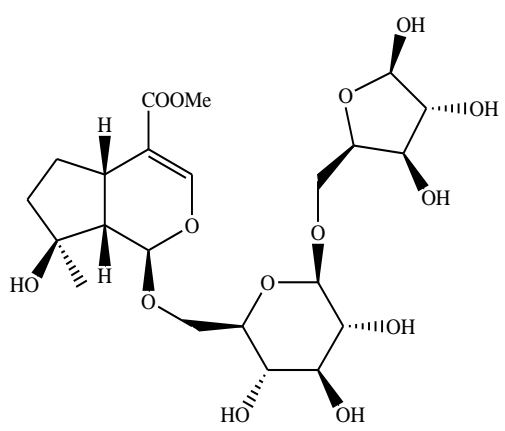

plicatoside B

Figure 17. Iridoids identified in Pedicularis species-part 3.<smiles>[R20]C1=CO[C@H]([R20])[C@]2(CC[C@@]2(C)O)[C@H]1[2H]</smiles>

$\mathrm{R}_{1}=\mathrm{Et}, \mathrm{R}_{2}=\mathrm{COOH}:$ pediverticilatasin $\mathrm{B}$ $\mathrm{R}_{1}=\mathrm{Et}, \mathrm{R}_{2}=$ CHO: pediverticilatasin C

$\mathrm{R}_{1}=\mathrm{Bu}, \mathrm{R}_{2}=\mathrm{COOH}$ : kansuenin $\mathrm{B}$

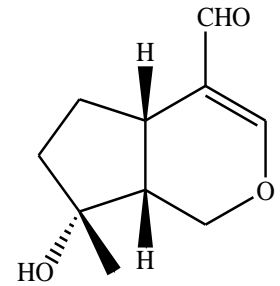

(rel-4aS,7R,7a $R$ )-1,4a,5,6,7,7ahexahydro-7hydroxyl-7-methyl-cyclopenta[c]pyran-4carboxaldehyde

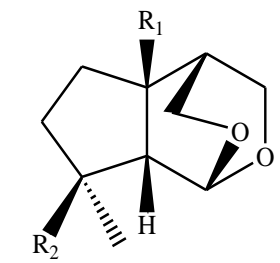

$\mathrm{R}_{1}=\mathrm{H}, \mathrm{R}_{2}=\mathrm{OH}$ : densispicnin $\mathrm{A}$ $\mathrm{R}_{1}=\mathrm{OH}, \mathrm{R}_{2}=\mathrm{H}$ : densispicnin $\mathrm{B}$

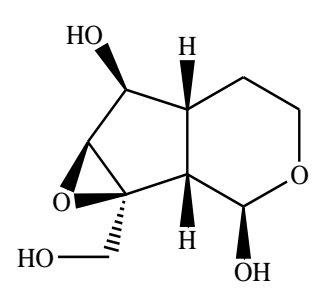

dihydro-catalpolgenin

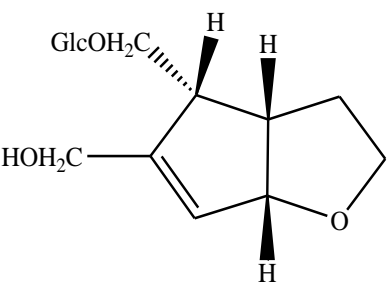

ningpogoside B<smiles>CCO[C@H]1OCC(=O)C2=C1[C@@](C)(O)CC2</smiles>

pediverticilatasin $\mathrm{A}$

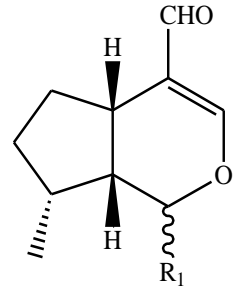

$\mathrm{R} 1=(R)-\mathrm{OMe}:$ artselaenin $\mathrm{A}$ $\mathrm{R} 1=(S)$-OMe: artselaenin B<smiles>[R3][C@@H]1OC=C(C=O)[C@H]2CC[C@](C)(O)[C@@H]12</smiles><smiles>C[C@H]1CC[C@H]2[C@H]1COC(=O)[C@@H]2C</smiles>

iridolactone

$\mathrm{R}_{1}=(S)-\mathrm{OH}:$ scyphiphin $\mathrm{A} 1$

$\mathrm{R}_{1}=(R)-\mathrm{OH}$ : scyphiphin $\mathrm{A} 2$

Figure 18. Iridoids identified in Pedicularis species-part 4. 
<smiles>CO[C@H]1OCC(C=O)=C2CCC(C)=C21</smiles>

1,3,5,6-tetrahydro-1-methoxyl-7-methylcyclopenta[c]pyran-4-carboxaldehyde

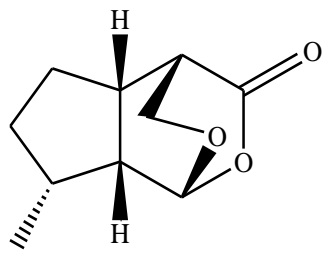

(rel-1R,4S,4aS,7R,7aR)-7-methyl-hexahydro-1,4(epoxymethano)-cyclopenta[c]pyran-3(1H)-one

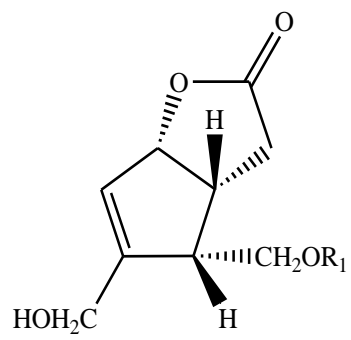

$\mathrm{R}_{1}=\mathrm{H}$ : pedicularislactone

$\mathrm{R}_{1}=\beta$-D-Glc: pedicularislactone-glucoside<smiles>C/C=C1\C(C(C)=O)=CC[C@H](OC(C)=O)[C@H]1CC(=O)OCCc1ccc(O)cc1</smiles>

ligustroside<smiles>C[C@]1(O)CCC2C1COC(=O)[C@@H]2Br</smiles>

$\mathrm{R}_{1}=\mathrm{H}: \operatorname{argyol}$

$\mathrm{R}_{1}=\mathrm{CH}_{2} \mathrm{OH}$ : mussaenin A<smiles>C[C@H]1CC[C@H](O)[C@H]1CO</smiles>

densispicnin D

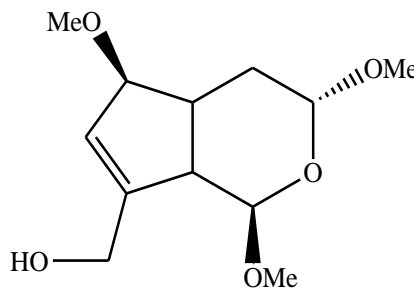

artselaenin III

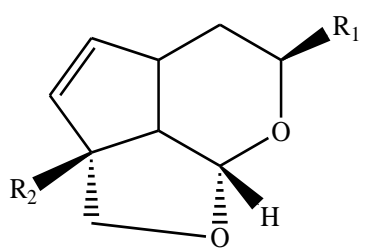

$\mathrm{R}_{1}=\mathrm{R}_{2}=$ OMe: artselaenin I $\mathrm{R}_{1}=\mathrm{R}_{2}=\mathrm{OH}$ : artselaenin $\mathrm{C}$
$\mathrm{R}_{1}=(S)-\mathrm{CH}_{2} \mathrm{OH}, \mathrm{R}_{2}=\mathrm{H}, \mathrm{R}_{3}=\mathrm{OH}$ : densispicnin $\mathrm{C}$

$\mathrm{R}_{1}=(S)-\mathrm{CH}_{2} \mathrm{OH}, \mathrm{R}_{2}=\mathrm{OH}, \mathrm{R}_{3}=\mathrm{H}$ : alyxialactone

$\mathrm{R}_{1}=(R)-\mathrm{CH}_{2} \mathrm{OH}, \mathrm{R}_{2}=\mathrm{OH}, \mathrm{R}_{3}=\mathrm{H}$ : 4-epialyxialactone

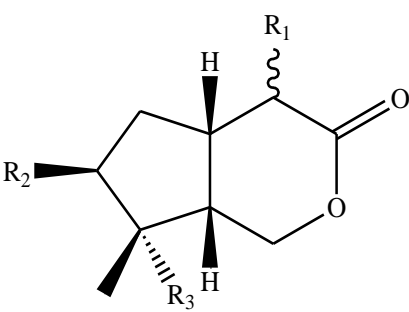

kansuenin

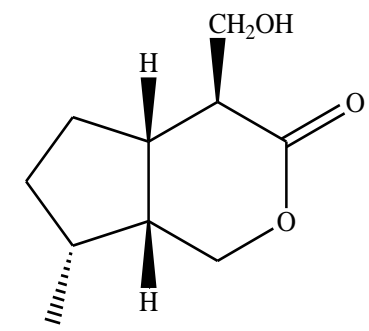

(4R)-4-hydroxymethyl-boschnialactone

Figure 19. Iridoids identified in Pedicularis species-part 5. 


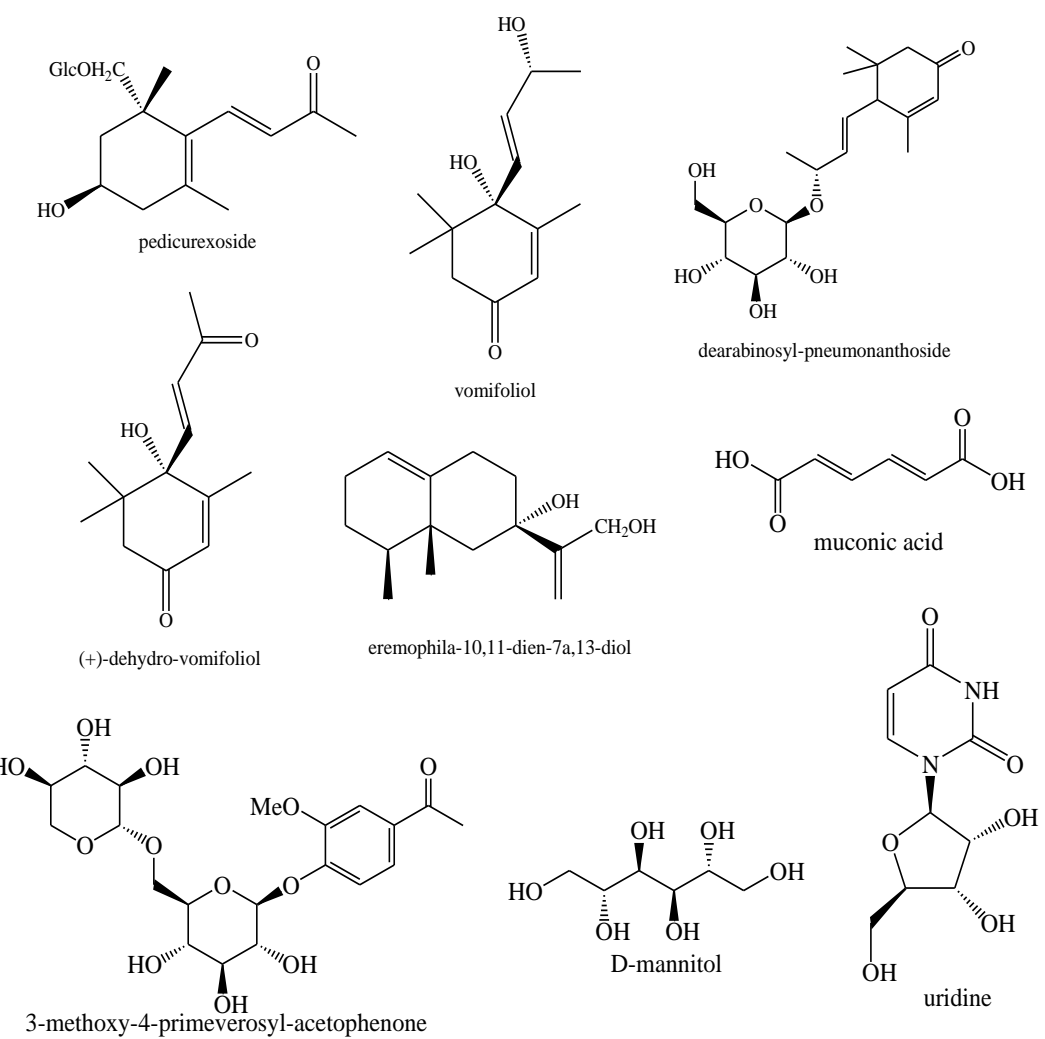

Figure 20. Other compounds identified in Pedicularis species-part 1.

$\mathrm{R}=\mathrm{H}$ : glycine

$\mathrm{R}=\mathrm{Me}$ : alanine

$\mathrm{R}=\mathrm{CH}_{2} \mathrm{COOH}$ : aspartic acid

$\mathrm{R}=\mathrm{CH}(\mathrm{OH}) \mathrm{CH}_{3}=$ threonine

$\mathrm{R}=\mathrm{CH}_{2} \mathrm{OH}$ : serine

$\mathrm{R}=\mathrm{CH}_{2} \mathrm{CH}_{2} \mathrm{COOH}$ : glutamic acid

$\mathrm{R}=\mathrm{CH}_{2} \mathrm{HS}$ : cysteine

$\mathrm{R}=\mathrm{CH}_{2} \mathrm{CH}_{2} \mathrm{SCH}_{3}$ : methionine

$\mathrm{R}=\mathrm{CH}\left(\mathrm{CH}_{3}\right) \mathrm{CH}_{2} \mathrm{CH}_{3}$ : isoleucine

$\mathrm{R}=\mathrm{CH}_{2}-\mathrm{C}_{6} \mathrm{H}_{5}$ : phenylalanine

$\mathrm{R}=\mathrm{CH}\left(\mathrm{CH}_{3}\right)_{2}$ : valine

$\mathrm{R}=\mathrm{CH}_{2} \mathrm{CH}_{2} \mathrm{CH}_{2} \mathrm{NHC}(\mathrm{NH}) \mathrm{NH}_{2}$ : arginine

$\mathrm{R}=\mathrm{CH}_{2} \mathrm{CH}\left(\mathrm{CH}_{3}\right)_{2}$ : leucine

$\mathrm{R}=\mathrm{CH}_{2}-p-\mathrm{OH}-\mathrm{C}_{6} \mathrm{H}_{4}$ : tyrosine<smiles>[R]c1cc(C(=O)O)c(Br)c([R9])c1[2H]</smiles>

$\mathrm{R}_{1}=\mathrm{OH}, \mathrm{R}_{2}=\mathrm{R}_{3}=\mathrm{R}_{4}=\mathrm{H}$ : salycilic acid

$\mathrm{R}_{1}=\mathrm{R}_{4}=\mathrm{OH}, \mathrm{R}_{2}=\mathrm{R}_{3}=\mathrm{H}: 2,5$-dihydroxybenzoic acid

$\mathrm{R}_{1}=\mathrm{R}_{4}=\mathrm{H}, \mathrm{R}_{2}=\mathrm{OH}, \mathrm{R}_{3}=\mathrm{OMe}$ : 3-hydroxy-4-methoxybenzoic acid

$\mathrm{R}_{1}=\mathrm{R}_{4}=\mathrm{H}, \mathrm{R}_{2}=\mathrm{OMe}, \mathrm{R}_{3}=\mathrm{OH}$ : 3-methoxy-4-hydroxybenzoic acid

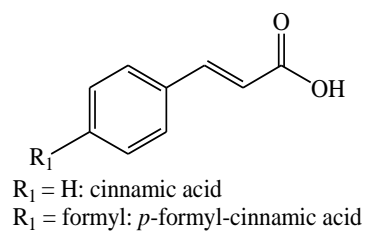<smiles>O=C(O)C1CCCN1</smiles>

proline

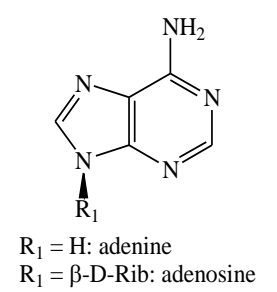

Figure 21. Other compounds identified in Pedicularis species-part 2.

\section{Corollary to Phytochemistry}

After visualization of the relative structures of the identified compounds in Pedicularis spp., two important elements must be observed and highlighted.

The first one concerns the compound found in P. kansuensis by Zhang et al. [50]. According to the structure, the compound should not be named as 1,2,3,16,19,20-hexahydroxyolean-12-en-28-oic acid, but rather as 1,2,3,16,19,20-hexahydroxy-12-ursen-28-oic acid on the basis of the vicinal dimethyl functionalization in positions 19,20 of the pentacyclic triterpene skeleton, which indicates it as an ursane and not an oleane. A similar observation is valid for the 1-(2,3,4-trihydroxyphenyl)ethyl-3-O -rhamnose-4-[(2E)-3-(3,4-dihydroxyphenyl)-2-propenoate]-glucopyranoside and 1-(2,3,4-trihydroxyph enyl)ethyl-3-O-rhamnose-4-[(2E)-3-(3,4-dihydroxyphenyl)-2-propenoate]-6-[(2E)-3-(3,4-dihydroxy-phen yl)-2-propenoate]-glucopyranoside identified in P. kansuesnsis $[49,50]$. These names were given by the 
authors, but, actually, according to the routinal numeration of this kind of compounds, they should be named as 1-(,3,4,5-trihydroxyphenyl)ethyl-3-O-rhamnose-4-[(2E)-3-(3,4-dihydroxyphenyl)-2-propen oate]-glucopyranoside and 1-(3,4,5-trihydroxyphenyl)ethyl-3-O-rhamnose-4-[(2E)-3-(3,4-dihydroxyph enyl)-2-propenoate]-6-[(2E)-3-(3,4-dihydroxyphenyl)-2-propenoate]-glucopyranoside, respectively.

Finally, there are some problems with the correct association between the name of the iridoid longifloroside and its structure, since diverse possibilities are given in the literature. Anyway, in this case, longifloroside is considered to be the compound with the

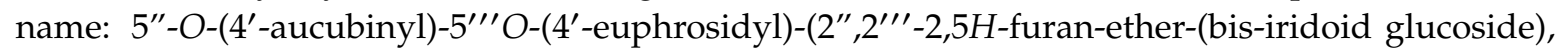
as reported in literature [55].

Moreover, for what concerns iridoids, some of those identified in Pedicularis spp. may indeed be artefacts due to the procedures applied during the phytochemical analysis. In particular, the two new iridoid glycosides 6-O-ethyl-aucubin and 6-O-ethyl-epi-aucubin, recognized from P. rex [69], and the three pediverticilatasins A-C isolated from $P$. verticillata [87], are likely due to the extraction with ethanol. The same has very likely happened for 6-O-methyl-aucubin, artselaenin III, and artselaenin I $[19,20]$, all isolated from P. artselaeri after extraction with boiling methanol (at reflux), as well as the 3-butoxy-3,4,dihydroaucubin, 6-O-butyl-aucubin, and 6-O-butyl-epi-aucubin obtained from the $n$-butanol soluble fraction of $P$. chinensis [27]. In this context, the ethyl acetal function observable in the pediverticilatasins A-C and the butyl acetal function of kansuenin B observed in P. verticillata [87] are also suspect, in particular if considering that the majority of these compounds have the alcoxy function of the acetal group in $\alpha$-configuration, which is the opposite of that generally observable for the saccharidic moieties in the glycosidic iridoids. Therefore, the presence of alcoxy acetals could possibly be due to an exchange between the saccharidic moiety and the alcohols present in solution as solvents (thus in high amount), favored by some specific conditions (i.e., acidity of the medium).

The possibility of generating this kind of artefacts from iridoids was one of the arguments of a recent review and of one editorial article $[89,90]$ which reported about the reactivity of the hydroxyl substituent in allylic configuration, a functionalization very often present in several iridoid structures, like in the case of iridoids with an aucubin-like skeleton, as well as the possibility of addition of short-chain alcohols used as extractive solvents to the double bond in the 3,4-positions of the iridane skeleton. Unfortunately, the presence of such iridoid derivatives was not confirmed in the studied species by avoiding the possible causes of artefact formation. Therefore, the presence of these compounds remains doubtful without any further confirmation.

\section{Methodologies for the Phytochemical Analysis}

The phytochemical analyses of the studied plants were carried out by following the common procedures. In particular, the essential oils were studied through hydrodistillation and gas chromatography (GC) and gas chromatography-mass spectrometry (GC-MS) analysis [10-13].

For the study of the polar fraction metabolites, the starting plant material was mainly dried. The extraction was mainly at room temperature with ethanol, even if extractions with different solvents such as methanol, $n$-hexane, and dichloromethane were also performed. In some cases, these latter extractions were carried out in hot conditions using a Soxhlet apparatus. This extraction method is not the best choice, since the exposure of the extracts to high temperatures may be one cause of artefact formation, as reported in the previous section. Indeed, extraction with ethanol was often followed by a partitioning procedure with solvents at different polarity grades, such as ethyl acetate, n-butanol, diethyl ether, petroleum ether, and distilled water, and every different organic phase was separately subjected to further analysis. The separation of the metabolites from the phytocomplex was mainly achieved by means of column chromatography (CC), using silica gel and allumina as stationary phases and different mixtures of $n$-butanol and distilled water, chloroform or dichloromethane and methanol, or $n$-hexane and ethyl acetate at different concentrations as mobile phases. In a few cases, high performance liquid chromatography (HPLC) techniques were used for these purposes, using C18 columns and distilled water and acetonitrile more or less acidified with formic acid as eluting systems. 
Identification of the metabolites was mainly achieved by means of thin layer chromatography (TLC), infrared (IR), ultraviolet (UV), optical rotation (OR), nuclear magnetic resonance (NMR), and mass spectrometry (MS) techniques. Finally, preliminary analysis of the metabolite contents was performed via the Folin-Cocalteau test for the total phenolic content (TPC), the aluminium chloride colorimetric assay for the total flavonoid content (TFC), Dragendorff's reagent test for the presence of alkaloids, and the ferric chloride test for the presence of tannins [15-88].

Nonetheless, in a few cases, the methodology was partially or totally undescribed in the reported experimental sections.

At this point, it is extremely important to underline two facts. The first is that the phytochemical methods employed for analysis can deeply influence the results. The second is that works performed only by chromatographic evidence and reporting the generic presence of classes of constituents cannot be considered totally reliable. An example of this is the Dragendorff's reagent test, which also results positive in the cases of $\alpha, \beta$-unsaturated carbonyls.

For these reasons, phytochemical methods must be carefully considered.

\section{Chemotaxonomy}

The chemotaxonomy of the Pedicularis genus is quite complex, and involves several classes of natural compounds. In particular, its main chemotaxonomic marker is aucubin, and, in fact, it has been recognized in 25 of the studied species (Table 1). From a biogenetic standpoint, aucubin, like the other decarboxylated C-10 iridoids observed in species of the Lamiales order, derives from geranyl pyrophosphate. In particular, these follow the biosynthetic Route II, which involves epi-iridotrial and 8-epi-deoxy-loganic acid among its precursors, and leads to the biosynthesis of iridoids characterized by the $\alpha$-configuration of the methyl function linked in the 8 position of the iridane skeleton. Its cyclization reaction occurs through a hydride nucleophillic attack on $\mathrm{C} 1$, which leads to the 1-O-carbonyl atom attack on C3 and then to the cyclic acetale [91]. Considering the biogenesis of iridoids in this genus, the actual presence of loganic acid recognized among the phytoconstituents of $P$. torta and P. longiflora is doubtful (see table for references). We are instead of the opinion that, without further confirmation, that compound was mistakenly reported instead of epi-loganic acid. In this context, studies on the biogenesis of iridoids in Pedicularis spp. by means of labelled precursors could be of help in delineating the biogenetic pathway and several products of that metabolite biosynthesis. This could also be an excellent analytical method by which to confirm the possible presence or not of compounds that appear to be in contrast with the biogenetic pathway of iridoids in this genus.

In fact, euphroside and mussaneoside are also minor chemotaxonomic markers of the genus, even if in several species the content of euphroside was shown to be higher than that of aucubin itself [22,51], and the amount of mussaenosidic acid was comparable with those of other iridoidic constituents [60]. Conversely, some iridoids are considered to be chemotaxonomic markers at the species level, since their presence has been reported only in one. The main example of this are pedicularioside for P. muscicola, kansuenin, kansuenin B, and kansuenoside for P. kansuensis, pliatosides A-B for P. plicata, and densispicnin A for P. densispica. In contrast with what is written in the previous paragraph concerning artefact iridoids, the presence of proceroside in P. procera [66], even if it presents a $\beta$-configuration in C-8 and therefore would seem to be derived from the Route I biogenetic pathway, is not an artefact and is not due to an erroneous interpretation of experimental data. In fact, the inversion of configuration at C-8 in proceroside is favored by the presence of a ketone function on the adjacent carbon (C-7), which is involved in a keto-enol equilibrium, and this may perfectly justify the $\beta$-configuration of the hydroxymethyl group at the position 8 .

Seco-iridoids are metabolites that rarely derive from the biogenetic Route II. In fact, some derivatives have been observed in Lamium album [92], and their origin from 8-epi-deoxy-loganic acid, a precursor in the biogenetic Route II, has been fully confirmed. To date, the presence of seco-iridoids has been observed only in P. verticillata [70], and it could be of utmost interest to verify if these kinds of 
compounds are also present in other species of the genus. Obviously, it could also be interesting to investigate their possible biogenesis by suitable analytical methods.

Phenylethanoid glycosides (i.e., verbascoside and its derivatives) are considered to be other chemotaxonomic markers of the genus, since their presence has been evidenced in most of the studied species. However, these compounds are very common in all the Asteridae class, and, in fact, they have also been identified in other families such as Asteraceae [93], Caprifoliaceae [94], Lamiaceae [14], Oleaceae [95], Plantaginaceae [96], Scrophulariaceae [97], and Verbenaceae [98]. More specifically, the phenylethanoid glycosides have a chemotaxonomical relevance when co-occurring with iridoids [99]. This has been already observed in several species in the Lamiales order [100-105], as well as in the case of several Pedicularis spp. These compounds are also extremely common within the family Pedicularis genus belongs to (Orobanchaceae), and, in fact, they have already been reported in several genera, such as Orobanche L., Cistanche L., and Orthocarpus Nutt. [106]. For these reasons, phenylethanoid glycosides cannot actually be taken as general chemotaxonomic markers of the Pedicularis genus. Nevertheless, specific compounds can be useful chemotaxonomic markers, such as pediculariosides A, E, G, H, I, M, and N for the entire genus, permethyl-verbascoside for P. spicata, cis-iso-martynoside for $P$. kansuensis, cis-pedicularioside $\mathrm{H}$ for P. spicata, and artselaeroside B for P. artselaeri.

Lignans and derivatives are quite widespread in the genus, but also in the family Orobanchaceae and in many others [107]. However, semitortosides A and B can serve as chemotaxonomic markers for P. semitorta, striatosides A and B can serve as chemotaxonomic markers for P. striata, and longiflor B and longiflorides $\mathrm{C}$ and $\mathrm{D}$ can serve as chemotaxonomic markers for P. longiflora.

Flavones and, in particular, flavonols and glycosidic flavonoids presenting an apigenin, scutellarein, and isoscutellarein base moiety, are also considered to be chemotaxonomic markers of the genus. However, they are very common compounds in the plant kingdom, and for this reason, they are not particularly useful as chemotaxonomic markers. In particular, their presence can be easily evidenced in Lamiaceae species [14], as well as in many other families, such as Euphorbiaceae, Asteraceae, Compositae, and Hypericaceae [108-114].

In terms of alkaloids, pediculidine, pedicularidine, pediculine, and pediculinine have been evidenced only in Pedicularis species, and they can serve as chemotaxonomic markers at the genus level.

As for compounds belonging to classes of natural metabolites other than the ones already described, there have been no reports on them as chemotaxonomic markers of the Pedicularis genus or in general, since they are extremely common. Nevertheless, pedicurexoside, a sesquiterpene, may be suggested as a specific marker for $P$. rex, since it has been evidenced only in that species so far, while the polyol D-mannitol seems to be highly represented in hemiparasitic entities previously comprised in Scrophulariaceae and now classified as Orobanchaceae [51,70,102,115].

In this context, concerning phytochemistry and chemotaxonomy, it is of primary importance to also consider other aspects, together with the markers' metabolite biogenesis, such as the ecology and hemiparasitic behaviour of the plant species, when the scope of the study is chemosystematics. In fact, the transfer of metabolites from the hosts to the hemiparasitic species has been observed in several cases, such in the cases of Euphrasia stricta D. Wolff [116], Euphrasia rostkoviana Hayne [117], and Odontites luteus Steven [118]. Therefore, it is suggested that the results from the phytochemical analysis of hemiparasitic plants should be carefully checked and subjected to the required criticism.

\section{Ethnopharmacology}

Pedicularis species are widely used in the traditional medicines of several countries around the world, especially Asian ones. The pharmacological activities exerted by these species are numerous and interesting, with one species often employed to treat more than one malady and vice versa.

Table 3 reports on the specific ethnopharmacological properties associated with every studied plant in this field. In addition, the organs of the plant species which show that medicinal activity are described, as well as the areas of the world where indigenous people employ these species in traditional medicine. 
Table 3. Ethnopharmacological Uses of Pedicularis Species as Reported in Literature.

\begin{tabular}{|c|c|c|c|c|}
\hline Pedicularis spp. & Ethnopharmacological Uses & Organ/Form & Area of the World & References \\
\hline P. artselaeri & $\begin{array}{l}\text { to treat diuresis, exhaustion, collapse, } \\
\text { senility }\end{array}$ & $\begin{array}{l}\text { aerial parts/ } \\
\text { n.r. }\end{array}$ & Northwestern China & [119] \\
\hline P. bicornuta & $\begin{array}{l}\text { - to treat vaginal and seminal discharges } \\
\text { - to treat burns, rheumatism, gout, } \\
\text { general inflammation, acidity }\end{array}$ & $\begin{array}{l}\text { - inflorescence/ } \\
\text { paste } \\
\text { - whole plant/ } \\
\text { decoction }\end{array}$ & $\begin{array}{l}\text { - Nepal (Central Himalaya) } \\
\text { - China, India }\end{array}$ & $\begin{array}{c}-[120] \\
-[8,121]\end{array}$ \\
\hline $\begin{array}{l}\text { P. bifida (Buch.-Ham.) Pennell } \\
\text { (u.n.) }\end{array}$ & $\begin{array}{l}\text { - to treat stomachache } \\
\text { - to relieve joint paints }\end{array}$ & $\begin{array}{l}\text { roots/ } \\
\text { liquid and powder }\end{array}$ & $\begin{array}{c}\text { Nepal (Newar community of } \\
\text { Pharping Village, Kathmandu } \\
\text { District) }\end{array}$ & [122] \\
\hline P. capitata & $\begin{array}{l}\text { - to sedate and relax } \\
\text { - to stop bleeding in minor injuries }\end{array}$ & $\begin{array}{l}\text { whole plant/ } \\
\text { infusion }\end{array}$ & $\begin{array}{c}\text { Canada (Inuit people of } \\
\text { Kugluktuk, Nunavut regions) }\end{array}$ & [123] \\
\hline P. cheilanthifolia Schrenk (a.n.) & $\begin{array}{l}\text { - to cure stomachache, vaginal discharge, } \\
\text { leucorrhoea, menorrhagia }\end{array}$ & $\begin{array}{l}\text { whole plant, wood/ } \\
\text { ethanolic extract, powder }\end{array}$ & $\begin{array}{l}\text { India/Kashmir } \\
\text { (Ladakh region) }\end{array}$ & {$[124,125]$} \\
\hline P. chenocephala Diels (a.n.) & $\begin{array}{l}\text { - to relieve pain } \\
\text { - to treat oedema, oliguria, asthma, } \\
\text { malnutrition, pains } \\
\text { induced by osteomyelitis }\end{array}$ & $\begin{array}{l}\text { flowers/ } \\
\text { decoction }\end{array}$ & China & [8] \\
\hline P. chinensis & $\begin{array}{l}\text { - to nourish yin } \\
\text { - to invigorate kidney } \\
\text { - to strengthen spleen and stomach }\end{array}$ & $\begin{array}{c}\text { roots/ } \\
\text { decoction }\end{array}$ & China & [8] \\
\hline P. comosa & - to be used as food stuff & $\begin{array}{l}\text { flowers/ } \\
\text { nectar }\end{array}$ & Turkey & [126] \\
\hline P. cranolopha Maxim. (a.n.) & $\begin{array}{l}\text { - to clear away heat evil } \\
\text { - to expel superficial evils } \\
\text { - to treat fever, urinary tract infections, } \\
\text { hepatitis, pneumonia, sore pain due to } \\
\text { external injury }\end{array}$ & $\begin{array}{l}\text { whole plant/ } \\
\text { decoction }\end{array}$ & China & [8] \\
\hline P. davidii Franch. (a.n.) & $\begin{array}{l}\text { - to strengthen spleen and stomach } \\
\text { - to nourish yin } \\
\text { - to relieve pain } \\
\text { - to treat inanition, kidney deficiency, } \\
\text { osteopyrexia, fever, joint pain, anorexia }\end{array}$ & $\begin{array}{l}\text { rhizomes/ } \\
\text { decoction }\end{array}$ & China & [8] \\
\hline
\end{tabular}


Table 3. Cont

\begin{tabular}{|c|c|c|c|c|}
\hline Pedicularis spp. & Ethnopharmacological Uses & Organ/Form & Area of the World & References \\
\hline P. decora & $\begin{array}{l}\text { - to treat general debility, collapse, } \\
\text { exhaustion, seminal emission, } \\
\text { spontaneous sweating and senility } \\
\text { - to invigorate the mind and the } \\
\text { circulation of blood } \\
\text { - to strengthen spleen and stomach }\end{array}$ & $\begin{array}{c}\text { roots/ } \\
\text { decoction }\end{array}$ & China & {$[8,127]$} \\
\hline P. decorissima Diels (a.n.) & $\begin{array}{l}\text { - to clear away heat evil } \\
\text { - to expel superficial evils } \\
\text { - to treat acute gastroenteritis and food } \\
\text { poisoning }\end{array}$ & $\begin{array}{l}\text { whole plant, flowers/ } \\
\text { decoction }\end{array}$ & China & [8] \\
\hline $\begin{array}{l}\text { P. dissecta (Bonati) Pennell \& } \\
\text { H.L. Li (a.n.) }\end{array}$ & $\begin{array}{l}\text { - to supplement qi } \\
\text { - to nourish yin } \\
\text { - to detoxificate } \\
\text { - to relieve pain } \\
\text { - to treat asthenia due to disease, yin } \\
\text { deficiency, sore, joint pains }\end{array}$ & $\begin{array}{c}\text { roots/ } \\
\text { decoction }\end{array}$ & China & [8] \\
\hline P. dunniana Bonati (a.n.) & $\begin{array}{l}\text { - to nourish yin } \\
\text { - to relieve pain } \\
\text { - to treat inanition, kidney deficiency, } \\
\text { osteopyrexia, fever, joint pains, anorexia }\end{array}$ & $\begin{array}{l}\text { rhizomes/ } \\
\text { decoction }\end{array}$ & China & [8] \\
\hline P. flagellaris Benth. (u.n.) & $\begin{array}{l}\text { - to treat excessive diuresis and wounds } \\
\text { - to treat excessive diuresis, wounds, } \\
\text { rheumatisms } \\
\text { - to regulate menstruation }\end{array}$ & $\begin{array}{c}\text { - aerial parts/ } \\
\text { infusion, decoction } \\
\text { - aerial parts/infusion, decoction }\end{array}$ & $\begin{array}{l}\text { - Himalaya } \\
\text { - Bhutan }\end{array}$ & $\begin{array}{l}-[128] \\
-[128]\end{array}$ \\
\hline P. flava Pall. (a.n.) & $\begin{array}{l}\text { - to treat general body pains, } \\
\text { stomachaches } \\
\text { - to be used as sedative }\end{array}$ & $\begin{array}{c}\text { leaves/ } \\
\text { decoction }\end{array}$ & Pakistan & [129] \\
\hline P. gracilis & to treat stomachache & $\begin{array}{l}\text { roots/ } \\
\text { liquid }\end{array}$ & $\begin{array}{l}\text { Nepal (Newar community of } \\
\text { Pharping village, Kathmandu } \\
\text { District; Western regions) }\end{array}$ & {$[122,130]$} \\
\hline
\end{tabular}


Table 3. Cont

\begin{tabular}{|c|c|c|c|c|}
\hline Pedicularis spp. & Ethnopharmacological Uses & Organ/Form & Area of the World & References \\
\hline P. gracilis subsp. gracilis (s.n.) & to relieve joint pain & $\begin{array}{l}\text { roots } \\
\text { /powder }\end{array}$ & Nepal (Central Himalaya) & [122] \\
\hline P. henryi Maxim. (a.n.) & $\begin{array}{l}\text { - to nourish yin and qi } \\
\text { - to strengthen tendons and bones with } \\
\text { vital essence } \\
\text { - to activate collaterals } \\
\text { - to treat hemiplegia and arthralgia due to } \\
\text { blood stagnation }\end{array}$ & $\begin{array}{c}\text { roots/ } \\
\text { decoction }\end{array}$ & China & [8] \\
\hline P. hoffmeisteri Klotzsch (a.n.) & $\begin{array}{l}\text { - to cure flatulence and stomach disorders } \\
\text { in animals } \\
\text { - to cure food poisoning }\end{array}$ & $\begin{array}{l}\text { - whole plant/ } \\
\text { n.r. } \\
\text { - whole plant/ } \\
\text { n.r. }\end{array}$ & $\begin{array}{c}\text { - India } \\
\text { (Uttaranchal State) } \\
\text { - India (Western Himalaya) }\end{array}$ & {$[131,132]$} \\
\hline P. integrifolia & $\begin{array}{l}\text { - to treat dropsy, excessive diuresis, } \\
\text { asthma, rheumatisms } \\
\text { - to heal wounds and oedema } \\
\text { - to nourish body }\end{array}$ & $\begin{array}{c}\text { aerial parts/ } \\
\text { ethanolic extract }\end{array}$ & Bhutan & [44] \\
\hline P. kansuensis & $\begin{array}{l}\text { - to treat collapse, exhaustion, senility, } \\
\text { edema and boils } \\
\text { - to relieve heat and toxicity } \\
\text { - to treat edema, inflammation, } \\
\text { urinaryobstructions }\end{array}$ & $\begin{array}{l}\text { - aerial parts/ } \\
\text { n.r. } \\
\text { - flowers/ } \\
\text { n.r. }\end{array}$ & $\begin{array}{c}\text { - China } \\
\text { - Tibet, China }\end{array}$ & $\begin{array}{l}-[50,119] \\
-[8]\end{array}$ \\
\hline $\begin{array}{c}\text { P. lanata Willd. ex Cham. \& } \\
\text { Schltdl. (a.n.) }\end{array}$ & to treat headache, migraine & n.r./n.r. & $\begin{array}{c}\text { Canada (Aborigens of the } \\
\text { Boreal forest) }\end{array}$ & [133] \\
\hline $\begin{array}{l}\text { P. longicaulis Franch. ex Maxim. } \\
\text { (a.n.) }\end{array}$ & $\begin{array}{l}\text { - to nourish yin and qi } \\
\text { - to activate collaterals } \\
\text { - to treat dizziness tinnitus, bones and } \\
\text { muscles pain, deficiency heat }\end{array}$ & roots/decoction & China & [8] \\
\hline
\end{tabular}


Table 3. Cont

\begin{tabular}{|c|c|c|c|c|}
\hline Pedicularis spp. & Ethnopharmacological Uses & Organ/Form & Area of the World & References \\
\hline P. longiflora & $\begin{array}{l}\text { - to cure hepatic, pancreatic, kidney, } \\
\text { urinary diseases, vaginal discharge, } \\
\text { leucorrhoea, menorrhagia } \\
\text { - to treat rheumatisms, excessive diuresis } \\
\text { and coagulation, wounds, hypertension, } \\
\text { dehydration } \\
\text { - to treat edema, tinnitus, carbuncles } \\
\text { wollen, hepatitis, spermatorrhea, urine } \\
\text { with pus and blood, cholecystitis, dry } \\
\text { mouth, carbuncle swollen } \\
\text { - to treat vertigo, dry tongue, excessive } \\
\text { seminal discharge, edema, liver and gall } \\
\text { bladder problems }\end{array}$ & $\begin{array}{l}\text { - whole plant, wood/ } \\
\text { decoction, powder } \\
\text { - aerial parts/ } \\
\text { ethanolic extract } \\
\text { - whole plant, flowers/ } \\
\text { decoction } \\
\text { - leaves, stems/ } \\
\text { decoctions }\end{array}$ & $\begin{array}{l}\text { - Himalaya (Ladakh region) } \\
\text { - Bhutan } \\
\text { - China } \\
\text { - India }\end{array}$ & $\begin{array}{l}-[7,125] \\
-[44] \\
-[8] \\
-[121]\end{array}$ \\
\hline P. longiflora var. tubiformis & $\begin{array}{l}\text { - to treat cough, sore throats, hepatitis, } \\
\text { lymphatic disorders, poisioning, seminal } \\
\text { and vaginal discharges, dropsy, } \\
\text { spermatorrhoea, tinnitus, carbuncle } \\
\text { disorders associated with alcoholism }\end{array}$ & $\begin{array}{l}\text { whole plant/ } \\
\text { raw food }\end{array}$ & Nepal (Central Himalaya) & {$[57,134]$} \\
\hline P. megalantha D.Don (a.n.) & $\begin{array}{l}\text { - to soothe meat poisoning, intestinal } \\
\text { disorders, acidity }\end{array}$ & $\begin{array}{l}\text { aerial parts/ } \\
\text { decoction }\end{array}$ & Bhutan, Tibet & [135] \\
\hline P. megalochila H.L. Li & $\begin{array}{l}\text { to treat dysentery, diarrhea, hepatitis, } \\
\text { urinary tract infections }\end{array}$ & $\begin{array}{l}\text { whole plant/ } \\
\text { decoction }\end{array}$ & China & [8] \\
\hline P. muscicola Maxim. (a.n.) & $\begin{array}{l}\text { - to nourish qi } \\
\text { - to treat consumptiondiseases, blood } \\
\text { deficiency, hidrosis, hypotension }\end{array}$ & $\begin{array}{c}\text { roots/ } \\
\text { decoction }\end{array}$ & China & [8] \\
\hline P. oederi Vahl (a.n.) & $\begin{array}{l}\text { - to treat rheumatic arthritis, lithangiuria, } \\
\text { scabies, micturition difficulties } \\
\text { - to treat food poisoning, headache, } \\
\text { backache, bodyache } \\
\text { - to be used as sedative }\end{array}$ & $\begin{array}{l}\text { - roots/ } \\
\text { decoction } \\
\text { - whole plant/ } \\
\text { raw vegetable }\end{array}$ & $\begin{array}{c}\text { - China } \\
\text { - India (Trans Himalaya region) }\end{array}$ & $\begin{array}{c}-[8] \\
-[121,136]\end{array}$ \\
\hline $\begin{array}{l}\text { P. oederi var. sinensis (Maxim.) } \\
\text { Hurus. (a.n.) }\end{array}$ & $\begin{array}{l}\text { to treat urinary obstructions and edema } \\
\text { in animals }\end{array}$ & $\begin{array}{l}\text { flowers/ } \\
\text { n.r. }\end{array}$ & Tibet, China & [137] \\
\hline
\end{tabular}


Table 3. Cont

\begin{tabular}{|c|c|c|c|c|}
\hline Pedicularis spp. & Ethnopharmacological Uses & Organ/Form & Area of the World & References \\
\hline P. oliveriana Prain (a.n.) & $\begin{array}{l}\text { - to reduce inflammation } \\
\text { - to ease gastric pains or disorders } \\
\text { - to treat poisoning, micturition } \\
\text { difficulties } \\
\text { - to cure food poisoning, stomach ulcer, } \\
\text { duodenal ulcer, diarrhea, rheumatic joint } \\
\text { pains, lithangiuria, abnormal leucorrhea, } \\
\text { scabies }\end{array}$ & $\begin{array}{c}\text { - inflorescence/ } \\
\text { extract } \\
\text { - flowers, whole plant/ } \\
\text { decoctions }\end{array}$ & $\begin{array}{c}\text { - Nepal (Central Himalaya) } \\
\text { - China }\end{array}$ & $\begin{array}{l}-[138] \\
-[8]\end{array}$ \\
\hline P. pectinata & $\begin{array}{l}\text { - to increase urine flow } \\
\text { - to cure swelling and stomach pains due } \\
\text { to intestinal infections } \\
\text { - to alleviate stomach pain, flatulence, } \\
\text { intestinal infections, intestinal swelling, } \\
\text { high blood pressure, backache, bodyache, } \\
\text { fever } \\
\text { - to increase urine flow } \\
\text { - to cure haemoptysis, alopecia }\end{array}$ & $\begin{array}{l}\text { - aerial parts/ } \\
\text { powdered raw food in cold } \\
\text { water } \\
\text { - flowers/ } \\
\text { powdered raw food in cold } \\
\text { water } \\
\text { - flowers/ } \\
\text { decoction }\end{array}$ & $\begin{array}{l}\text { - Kashmir } \\
\text { - Western Himalaya } \\
\text { (Lahaul-Spiti tribe) } \\
\text { - India, Kashmir }\end{array}$ & $\begin{array}{c}-[139] \\
-[140] \\
-[141-143]\end{array}$ \\
\hline P. pectinatiformis Bonati (a.n.) & $\begin{array}{l}\text { - to relieve pain } \\
\text { - to relax }\end{array}$ & $\begin{array}{c}\text { leaves/ } \\
\text { infusion }\end{array}$ & $\begin{array}{c}\text { Pakistan (Gilgit-Baltistan } \\
\text { region) }\end{array}$ & [144] \\
\hline P. peduncularis & $\begin{array}{l}\text { - to treat uterine bleeding } \\
\text { - to favour diuresis } \\
\text { - to treat various skin diseases }\end{array}$ & $\begin{array}{c}\text { - aerial parts/ } \\
\text { decoction } \\
\text { - flowers/ } \\
\text { decoction } \\
\text { - aerial parts/ } \\
\text { bath }\end{array}$ & Tajikistan & [64] \\
\hline P. punctata & $\begin{array}{l}\text { - to treat fever, cancer and premature } \\
\text { graying of hair } \\
\text { - to improve digestion } \\
\text { - to control blood pressure } \\
\text { - to treat hypertension, fever, } \\
\text { gastrointestinal disorders } \\
\text { - to relax skeletal muscles }\end{array}$ & $\begin{array}{c}\text { - inflorescence/ } \\
\text { extract } \\
\text { - aerial parts/powdered raw } \\
\text { food in cold water } \\
\text { - flowers/ } \\
\text { powder in cold water }\end{array}$ & $\begin{array}{c}\text { - Nepal (Central Himalaya) } \\
\text { - Western Himalaya } \\
\text { (Lahaul-Spiti tribe) } \\
\text { - Pakistan }\end{array}$ & $\begin{array}{c}-[138] \\
-[140,145] \\
-[146,147]\end{array}$ \\
\hline
\end{tabular}


Table 3. Cont

\begin{tabular}{|c|c|c|c|c|}
\hline Pedicularis spp. & Ethnopharmacological Uses & Organ/Form & Area of the World & References \\
\hline $\begin{array}{l}\text { P. pyramidata Royle ex Benth. } \\
\text { (a.n.) }\end{array}$ & $\begin{array}{l}\text { to treat fluid retention, headache, bone } \\
\text { inflammations, serous fluids } \\
\text { accumulation }\end{array}$ & $\begin{array}{l}\text { whole plant/ } \\
\text { raw food }\end{array}$ & Nepal (Central Himalaya), India & {$[138,143]$} \\
\hline P. resupinata & $\begin{array}{l}\text { - to treat malignant abscesses } \\
\text { - to treat rheumatoid arthritis, rheumatic } \\
\text { pains, joint pains, scabies, micturition } \\
\text { difficulties } \\
\text { - to cure lithangiuria abnormal leucorrhea, } \\
\text { acute gastroenteritis, food poisoning }\end{array}$ & $\begin{array}{l}\text { - aerial parts/n.r. } \\
\text { - roots, stem/ } \\
\text { powder, decoctions }\end{array}$ & $\begin{array}{l}\text { - South Korea } \\
\text { - China }\end{array}$ & $\begin{array}{l}-[148] \\
-[8]\end{array}$ \\
\hline P. rex & $\begin{array}{l}\text { - to invigorate qi and blood } \\
\text { - to strengthen spleen } \\
\text { - to treat yin deficiency, hectic fever, } \\
\text { rheumatism, cirrhosis, ascites } \\
\text { - to cure smallpox, measles, seasonal } \\
\text { prevalent diseases }\end{array}$ & $\begin{array}{l}\text { roots, whole plant/ } \\
\text { decoctions }\end{array}$ & China & [8] \\
\hline P. rhinanthoides Schrenk (a.n.) & $\begin{array}{l}\text { - to treat cough, sore throat, hepatitis, } \\
\text { lymphatic disorders, poisoning } \\
\text { - to treat diabetes }\end{array}$ & $\begin{array}{l}\text { whole plant/ } \\
\text { raw food } \\
\text { - whole plant/ } \\
\text { decoction }\end{array}$ & $\begin{array}{c}\text { - Nepal (Central Himalaya) } \\
\text { - India }\end{array}$ & $\begin{array}{l}-[138] \\
-[149]\end{array}$ \\
\hline P. rudis Maxim. (a.n.) & $\begin{array}{l}\text { - to nourish yin } \\
\text { - to relieve pain } \\
\text { - to treat inanition, kidney deficiency, } \\
\text { osteopyrexia, fever, joint pain, anorexia }\end{array}$ & $\begin{array}{l}\text { rhizomes/ } \\
\text { decoction }\end{array}$ & China & [8] \\
\hline $\begin{array}{l}\text { P. scullyana Prain ex Maxim. } \\
\text { (u.n.) }\end{array}$ & to remove pimples & $\begin{array}{l}\text { whole plant/ } \\
\text { paste }\end{array}$ & Nepal (Western regions) & [130] \\
\hline P. siphonantha & $\begin{array}{l}\text { to treat cough, sore throat, hepatitis, } \\
\text { lymphatic disorders, poisoning }\end{array}$ & $\begin{array}{l}\text { whole plant/ } \\
\text { raw food }\end{array}$ & Nepal (Central Himalaya) & {$[134,150]$} \\
\hline
\end{tabular}


Table 3. Cont

\begin{tabular}{|c|c|c|c|c|}
\hline Pedicularis spp. & Ethnopharmacological Uses & Organ/Form & Area of the World & References \\
\hline P. spicata & $\begin{array}{l}\text { - to nourish qi } \\
\text { - to treat consumption } \\
\text { diseases, blood deficiency, hidrosis, } \\
\text { hypotension }\end{array}$ & roots/decoction & China & [8] \\
\hline P. striata & $\begin{array}{l}\text { to treat kidney-yang deficiency, edema, } \\
\text { micturition difficulties }\end{array}$ & $\begin{array}{l}\text { whole plant/ } \\
\text { decoction }\end{array}$ & China & [8] \\
\hline P. tenuirostris & $\begin{array}{l}\text { to cure swelling and stomach pain due to } \\
\text { intestinal infections }\end{array}$ & $\begin{array}{c}\text { flowers/ } \\
\text { powdered raw food in cold } \\
\text { water }\end{array}$ & $\begin{array}{c}\text { Western Himalaya (Lahaul-Spiti } \\
\text { tribe) }\end{array}$ & [140] \\
\hline P. torta & $\begin{array}{l}\text { to treat inflammations and urinary } \\
\text { obstructions in animals }\end{array}$ & $\begin{array}{l}\text { flowers/ } \\
\text { n.r. }\end{array}$ & Tibet, China & [137] \\
\hline P. verticillata & $\begin{array}{l}\text { - to nourish qi } \\
\text { - to treat consumption } \\
\text { diseases, blood deficiency, hidrosis, } \\
\text { hypotension }\end{array}$ & roots/decoction & China & [8] \\
\hline
\end{tabular}




\section{Corollary for Ethnopharmacology}

Some Pedicularis species have also been reported to have ethnopharmacological employments in certain areas of the world, but no specific medicinal and pharmacological properties have been reported in the literature. In particular, this concerns P. koengboensis Tsoong var. kongboensis (a.n.) in Nepal [151], P. heydei Prain (u.n.), P. nodosa Pennell (u.n.) and P. scullyana Prain ex. Maxim. (u.n.) in Tibet [152], and, finally, P. tristis L. (a.n.) in Mongolia [153]. Specific information concerning their specific way of employment is also lacking in the literature, which makes their uses doubtful but not certainly false, since their utilization may be only on a traditional local basis and favored by specialized people who may not be interested in sharing their knowledge. Regardless, phytochemical analysis of these species is also strongly suggested in the future.

\section{Pharmacology}

In spite of all the results reported in the previous section, for some Pedicularis species, only a few initial pharmacological properties have been assessed, and their ethnopharmacological employments have not yet been reported. This also concerns the species already used in the ethnopharmacological field but that have been studied for other possible employments.

Table 4 reports on these species and their relative pharmacological properties.

Table 4. Pharmacological Activities of Pedicularis Species as Reported in Literature.

\begin{tabular}{|c|c|c|c|c|}
\hline Pedicularis spp. & $\begin{array}{l}\text { Pharmacological } \\
\text { Properties }\end{array}$ & Organs/Forms & Collection Area & References \\
\hline P. artselaeri & $\begin{array}{l}\text { - strong antioxidant } \\
\text { - hepatoprotective }\end{array}$ & $\begin{array}{c}\text { - aerial parts/ } \\
\text { butanol and water } \\
\text { extracts } \\
\text { - water and } \\
\text { ethanolic extracts }\end{array}$ & China & $\begin{array}{l}-[154] \\
-[155]\end{array}$ \\
\hline $\begin{array}{l}\text { P. cadmea Boiss. } \\
\text { (u.n.) }\end{array}$ & weak antibacterial & $\begin{array}{c}\text { aerial parts/ } \\
\text { methanolic extract }\end{array}$ & Turkey & [156] \\
\hline P. condensata & $\begin{array}{l}\text { antibacterial, weak } \\
\text { antioxidant, antifungal }\end{array}$ & $\begin{array}{l}\text { aerial parts/ } \\
\text { essential oil }\end{array}$ & Turkey & [10] \\
\hline P. davidii & $\begin{array}{l}\text { - strong antioxidant } \\
\text { - hepatoprotective }\end{array}$ & $\begin{array}{c}\text { - rhizomes/ } \\
\text { butanol and water } \\
\text { extracts } \\
\text { - water and } \\
\text { ethanolic extracts }\end{array}$ & China & $\begin{array}{l}-[154] \\
-[155]\end{array}$ \\
\hline P. decora & $\begin{array}{l}\text { antioxidant, antidiabetic, } \\
\text { hepatoprotective, } \\
\text { anti-inflammatory }\end{array}$ & $\begin{array}{l}\text { roots/ethanolic, } \\
\text { n-butanol and } \\
\text { water extracts }\end{array}$ & China & {$[7,157,158]$} \\
\hline P. flava & medium antimicrobial & $\begin{array}{l}\text { whole plant/ } \\
\text { ethanolic extract }\end{array}$ & Mongolia & [159] \\
\hline P. longiflora & $\begin{array}{l}\text { antidiabetic, antioxidant, } \\
\text { radical scavenging }\end{array}$ & $\begin{array}{l}\text { whole plant/ } \\
\text { ethanolic extract }\end{array}$ & $\begin{array}{l}\text { Himalaya (Ladakh } \\
\text { region), China }\end{array}$ & {$[7,56]$} \\
\hline $\begin{array}{l}\text { P. olympica Boiss. } \\
\text { (u.n.) }\end{array}$ & weak antimicrobial & $\begin{array}{c}\text { aerial parts/ } \\
\text { methanolic extract }\end{array}$ & Turkey & [155] \\
\hline $\begin{array}{l}\text { P. mexicana Zucc. } \\
\text { ex Bunge (a.n.) }\end{array}$ & $\begin{array}{l}\text { antioxidant, medium } \\
\text { cytotoxic }\end{array}$ & $\begin{array}{c}\text { whole plant/ } \\
\text { methanolic extract }\end{array}$ & Mexico & [160] \\
\hline P. sibthorpii & $\begin{array}{l}\text { strong antioxidant, } \\
\text { free-radical scavenging, } \\
\text { antibacterial }\end{array}$ & $\begin{array}{c}\text { aerial parts/ } \\
\text { methanolic extract }\end{array}$ & Iran & [74] \\
\hline P. wilhelmsiana & $\begin{array}{l}\text { strong antioxidant, } \\
\text { antibacterial }\end{array}$ & $\begin{array}{c}\text { aerial parts/ } \\
\text { methanolic extract }\end{array}$ & Iran & [12] \\
\hline
\end{tabular}




\section{Relationships among Pharmacology, Ethnopharmacology, and Phytochemistry}

Tables 2 and 3 clearly show how fundamental Pedicularis species are in the ethnopharmacological and pharmacological fields. However, many Pedicularis species with ethnopharmacological and/or pharmacological uses are awaiting phytochemical analysis on their active constituents. Thus, their employment is strictly related to traditional uses, which are established on the basis of previous experiences. Conversely, for those species also presenting a well-established phytochemical profile, their ethnopharmacological and/or pharmacological uses can be obviously explained by their phytochemical compositions. In fact, phytochemical compounds (singularly or as a phytocomplex) are the major elements responsible for the pharmacological properties associated to every single species, and may justify their use in that sense from the phytochemical standpoint.

Several classes of natural compounds have been evidenced within the Pedicularis genus, and each of them exerts specific pharmacological activities. In particular, alkaloids have antimalarial, antitumor, antibacterial, and stimulant activities, among others [154,161], even if a particular subclass of them (pyrrolizidine alkaloids) are indeed known to cause severe genotoxicity, neurotoxicity, and tumourigenicity [162]. Lignans exert mainly antioxidant and anti-inflammatory properties [163]. Tannins are widely known for their astringent and antioxidant effects [164]. Phenylethanoid glycosides are good antioxidant, antibacterial, antiviral, antitumor, neuroprotective, and hepatoprotective compounds [106,165]. Flavonoids display, in particular, antioxidant, anti-inflammatory, anti-mutagenic, and anti-carcinogenic properties [166]. Xanthones are mainly insecticidal compounds [167]. Iridoids are widely used as antiviral, anti-inflammatory, hepatoprotective, antimicrobial, and antitumor agents [168]. Seco-iridoids are mainly anti-inflammatory and antifungal compounds [169]. Finally, fatty acids, organic acids, polyols, saccharides, nucleobases, and amino acids have several nutraceutical properties.

\section{Other Uses}

Some Pedicularis species are better known to have other uses different from those typical in the ethnopharmacological and pharmacological fields.

These uses all are reported in the table below (Table 5).

Table 5. Other Uses of Pedicularis Species As Reported In Literature.

\begin{tabular}{|c|c|c|c|c|}
\hline Pedicularis spp. & Other Uses & Organs/Forms & Area of Employment & References \\
\hline $\begin{array}{l}\text { P. atuntsiensis Bonati } \\
\text { (a.n.) }\end{array}$ & purely ornamental & - & $\begin{array}{c}\text { China (Northwestern } \\
\text { Yunnan) }\end{array}$ & [170] \\
\hline P. capitata & $\begin{array}{l}\text { to make an olive } \\
\text { green dye }\end{array}$ & flower stalks & $\begin{array}{c}\text { Canada (Inuit people of } \\
\text { Kugluktuk, Nunavut } \\
\text { regions) }\end{array}$ & [123] \\
\hline $\begin{array}{l}\text { P. crenularis H.L. Li } \\
\text { (a.n.) }\end{array}$ & purely ornamental & - & $\begin{array}{c}\text { China (Northwestern } \\
\text { Yunnan) }\end{array}$ & [170] \\
\hline $\begin{array}{l}\text { P. cyclorhyncha H.L. Li } \\
\text { (a.n.) }\end{array}$ & purely ornamental & - & $\begin{array}{c}\text { China (Northwestern } \\
\text { Yunnan) }\end{array}$ & [170] \\
\hline $\begin{array}{c}\text { P. dichrocephala } \\
\text { Hand.-Mazz. (a.n.) }\end{array}$ & purely ornamental & - & $\begin{array}{c}\text { China (Northwestern } \\
\text { Yunnan) }\end{array}$ & [170] \\
\hline $\begin{array}{l}\text { P. fastigiata Franch. } \\
\text { (a.n.) }\end{array}$ & purely ornamental & - & $\begin{array}{c}\text { China (Northwestern } \\
\text { Yunnan) }\end{array}$ & [170] \\
\hline $\begin{array}{l}\text { P. filicula Franch. ex. } \\
\text { Maxim. (a.n.) }\end{array}$ & purely ornamental & - & $\begin{array}{c}\text { China (Northwestern } \\
\text { Yunnan) }\end{array}$ & [170] \\
\hline P. flava & forage & - & Pakistan & [129] \\
\hline $\begin{array}{l}\text { P. gracilicaulis H.L. Li } \\
\text { (a.n.) }\end{array}$ & purely ornamental & - & $\begin{array}{c}\text { China (Northwestern } \\
\text { Yunnan) }\end{array}$ & [170] \\
\hline
\end{tabular}


Table 5. Cont.

\begin{tabular}{|c|c|c|c|c|}
\hline Pedicularis spp. & Other Uses & Organs/Forms & Area of Employment & References \\
\hline P. groenlandica & edible plant & $\begin{array}{l}\text { whole } \\
\text { plant/tea }\end{array}$ & $\begin{array}{c}\text { Canada (Inuit people, } \\
\text { Kangiqsualujjuaq } \\
\text { community) }\end{array}$ & [171] \\
\hline $\begin{array}{l}\text { P. habachanensis Bonati } \\
\text { (a.n.) }\end{array}$ & purely ornamental & - & $\begin{array}{c}\text { China (Northwestern } \\
\text { Yunnan) }\end{array}$ & [170] \\
\hline P. humilis Bonati (a.n.) & purely ornamental & - & $\begin{array}{c}\text { China (Northwestern } \\
\text { Yunnan) }\end{array}$ & [170] \\
\hline P. kariensis Bonati (a.n.) & purely ornamental & - & $\begin{array}{c}\text { China (Northwestern } \\
\text { Yunnan) }\end{array}$ & [170] \\
\hline $\begin{array}{l}\text { P. labradorica Wirsing } \\
\text { (a.n.) }\end{array}$ & edible plant & roots & $\begin{array}{l}\text { Canada (Inuit people, } \\
\text { Nain community) }\end{array}$ & [170] \\
\hline $\begin{array}{c}\text { P. lamioides } \\
\text { Hand.-Mazz. (a.n.) }\end{array}$ & purely ornamental & - & $\begin{array}{c}\text { China (Northwestern } \\
\text { Yunnan) }\end{array}$ & [169] \\
\hline $\begin{array}{l}\text { P. lanpingensis H.P. } \\
\text { Yang (a.n.) }\end{array}$ & purely ornamental & - & $\begin{array}{c}\text { China (Northwestern } \\
\text { Yunnan) }\end{array}$ & [169] \\
\hline P. lecomtei Bonati (a.n.) & purely ornamental & - & $\begin{array}{c}\text { China (Northwestern } \\
\text { Yunnan) }\end{array}$ & [169] \\
\hline $\begin{array}{l}\text { P. macrorhyncha H.L. Li } \\
\text { (a.n.) }\end{array}$ & purely ornamental & - & $\begin{array}{c}\text { China (Northwestern } \\
\text { Yunnan) }\end{array}$ & [170] \\
\hline P. maxonii Bonati (a.n.) & purely ornamental & - & $\begin{array}{c}\text { China (Northwestern } \\
\text { Yunnan) }\end{array}$ & [170] \\
\hline $\begin{array}{l}\text { P. mayana Hand.-Mazz. } \\
\text { (a.n.) }\end{array}$ & purely ornamental & - & $\begin{array}{c}\text { China (Northwestern } \\
\text { Yunnan) }\end{array}$ & [170] \\
\hline $\begin{array}{l}\text { P. meteororhyncha H.L. } \\
\text { Li (a.n.) }\end{array}$ & purely ornamental & - & $\begin{array}{c}\text { China (Northwestern } \\
\text { Yunnan) }\end{array}$ & [170] \\
\hline $\begin{array}{l}\text { P. micrantha H.L. Li } \\
\text { (a.n.) }\end{array}$ & purely ornamental & - & $\begin{array}{c}\text { China (Northwestern } \\
\text { Yunnan) }\end{array}$ & [170] \\
\hline $\begin{array}{l}\text { P. mussotii Franch. } \\
\text { (a.n.) }\end{array}$ & purely ornamental & - & $\begin{array}{c}\text { China (Northwestern } \\
\text { Yunnan) }\end{array}$ & [170] \\
\hline P. obscura Bonati (a.n.) & purely ornamental & - & $\begin{array}{c}\text { China (Northwestern } \\
\text { Yunnan) }\end{array}$ & [170] \\
\hline P. oederi & fodder & $\begin{array}{l}\text { whole } \\
\text { plant/raw } \\
\text { food }\end{array}$ & Nepal (Central Himalaya) & [138] \\
\hline $\begin{array}{l}\text { P. oligantha Franch. ex. } \\
\text { Maxim. (a.n.) }\end{array}$ & purely ornamental & - & $\begin{array}{c}\text { China (Northwestern } \\
\text { Yunnan) }\end{array}$ & [170] \\
\hline $\begin{array}{l}\text { P. orthocoryne H.L. Li } \\
\text { (a.n.) }\end{array}$ & purely ornamental & - & $\begin{array}{c}\text { China (Northwestern } \\
\text { Yunnan) }\end{array}$ & [170] \\
\hline $\begin{array}{c}\text { P. pinetorum } \\
\text { Hand.-Mazz. (a.n.) }\end{array}$ & purely ornamental & - & $\begin{array}{c}\text { China (Northwestern } \\
\text { Yunnan) }\end{array}$ & [170] \\
\hline $\begin{array}{l}\text { P. praeruptorum Bonati } \\
\text { (a.n.) }\end{array}$ & purely ornamental & - & $\begin{array}{c}\text { China (Northwestern } \\
\text { Yunnan) }\end{array}$ & [170] \\
\hline $\begin{array}{l}\text { P. pseudoversicolor } \\
\text { Hand.-Mazz. (a.n.) }\end{array}$ & purely ornamental & - & $\begin{array}{c}\text { China (Northwestern } \\
\text { Yunnan) }\end{array}$ & [170] \\
\hline $\begin{array}{c}\text { P. remotiloba } \\
\text { Hand.-Mazz. (a.n.) }\end{array}$ & purely ornamental & - & $\begin{array}{c}\text { China (Northwestern } \\
\text { Yunnan) }\end{array}$ & [170] \\
\hline P. salicifolia Bonati (a.n.) & purely ornamental & - & $\begin{array}{c}\text { China (Northwestern } \\
\text { Yunnan) }\end{array}$ & [170] \\
\hline
\end{tabular}


Table 5. Cont.

\begin{tabular}{|c|c|c|c|c|}
\hline Pedicularis spp. & Other Uses & Organs/Forms & Area of Employment & References \\
\hline $\begin{array}{l}\text { P. schizocalyx (Lange) } \\
\text { Steininger (a.n.) }\end{array}$ & edible & $\begin{array}{c}\text { flowers/raw } \\
\text { plant }\end{array}$ & Spain (Cantabria region) & [172] \\
\hline $\begin{array}{l}\text { P. sigmoidea Franch. ex. } \\
\text { Maxim. (a.n.) }\end{array}$ & purely ornamental & - & $\begin{array}{c}\text { China (Northwestern } \\
\text { Yunnan) }\end{array}$ & [170] \\
\hline P. sylvatica & edible & $\begin{array}{c}\text { flowers/raw } \\
\text { plant }\end{array}$ & Spain (Galicia region) & [170] \\
\hline $\begin{array}{l}\text { P. tomentosa H.L. Li } \\
\text { (a.n.) }\end{array}$ & purely ornamental & - & $\begin{array}{c}\text { China (Northwestern } \\
\text { Yunnan) }\end{array}$ & [170] \\
\hline P. tsaii H.L. Li (a.n.) & purely ornamental & - & $\begin{array}{c}\text { China (Northwestern } \\
\text { Yunnan) }\end{array}$ & [170] \\
\hline $\begin{array}{l}\text { P. umbelliformis H.L. Li } \\
\text { (a.n.) }\end{array}$ & purely ornamental & - & $\begin{array}{c}\text { China (Northwestern } \\
\text { Yunnan) }\end{array}$ & [170] \\
\hline $\begin{array}{l}\text { P. weixiensis H.P. Yang } \\
\text { (a.n.) }\end{array}$ & purely ornamental & - & $\begin{array}{c}\text { China (Northwestern } \\
\text { Yunnan) }\end{array}$ & [170] \\
\hline P. yui H.L. Li (a.n.) & purely ornamental & - & $\begin{array}{c}\text { China (Northwestern } \\
\text { Yunnan) }\end{array}$ & [170] \\
\hline $\begin{array}{c}\text { P. zhongdianensis H.P. } \\
\text { Yang (a.n.) }\end{array}$ & purely ornamental & - & $\begin{array}{c}\text { China (Northwestern } \\
\text { Yunnan) }\end{array}$ & [170] \\
\hline
\end{tabular}

\section{Curiosities}

Some Pedicularis species present strange but interesting curiosities. In particular, although Pedicularis species are considered to be strong hemiparasitic plants, P. friderici-augusti Tomm. (a.n.), P. furbishiae S. Watson (a.n.), P. ishidoyana Koidz. \& Ohwi (u.n.), P. kashmiriana Pennell (a.n.), P. petiolaris Ten. (a.n.), P. rainierensis Pennel \& Warren (a.n.), P. rostratospicata Crantz (a.n.), P. siamensis P.C.Tsoong (u.n.), and P. thailandica T.Yamaz. (u.n.) are endangered species in their growth areas [173-179]. Moreover, P. porrecta Wall. (u.n.) grows only in arid areas [180], and the name P. stenantha Franch. (u.n.) is also often used to identify P. stenocorys Franch. (a.n.), but they are two different species [181].

\section{Conclusions}

This review has clearly evidenced and highlighted the importance of the plant species belonging to the Pedicularis genus from different points of view.

As it can be easily deduced, there is still much to discover and study, since the information about this genus is quite scarce as regards many specific arguments.

In particular, it could be interesting to investigate the biogenesis of iridoids, since, from a chemosystematic standpoint, they are the most important marker compounds in this genus. This may confirm or not the presence of unusual compounds such as derivatives with $8 \beta$-configurations and seco-iridoids, as well as potentially elucidate the key intermediates in their biosynthesis by means of labeled precursors.

On the other hand, for what concerns the bioactivity aspects of Pedicularis spp., we hope that this review will contribute to renewing the interest of researchers in deepening the general knowledge on the pharmacological potentials of Pedicularis extracts and pure constituents, in particular, their minor components.

Author Contributions: Conceptualization, C.F., A.B. and M.S.; All authors wrote and reviewed every section.

Funding: This research received no external funding.

Conflicts of Interest: The authors declare no potential conflict of interests in this work. 


\section{Abbreviations}

$\begin{array}{ll}\text { a.n. } & \text { accepted name } \\ \text { n.r. } & \text { none reported } \\ \text { n.s. } & \text { not specified } \\ \text { s.n. } & \text { synonym name } \\ \text { u.n. } & \text { unresolved name }\end{array}$

\section{References}

1. The Angiosperm Phylogeny Group. An update of the Angiosperm Phylogeny Group classification for the orders and families of flowering plants: APG IV. Bot. J. Linn. Soc. 2016, 181, 1-20. [CrossRef]

2. Pedicularis genus. Available online: www.theplantlist.org (accessed on 15 May 2019).

3. Nicolini, G. Enciclopedia Botanica Motta; Federico Motta: Milano, Italy, 1960; Volume 3, p. 236.

4. $\quad$ Pignatti, S. Flora d'Italia; Edagricole: Bologna, Italy, 1982; Volume 2, p. 590.

5. Kadereit, J.W. The Families and Genera of Vascular Plants, Volume VII; Lamiales Sperling: Berlin, Germany, $2004 ;$ p. 424.

6. Aeschimann, D.; Lauber, K.; Moser, D.M.; Theurillat, J.P. Flora Alpina; Zanichelli: Bologna, Italy, 2004; Volume 2, p. 264.

7. Yatoo, M.I.; Dimri, U.; Gopalakrishan, A.; Saminathan, M.; Dhama, K.; Mathesh, K.; Saxena, A.; Gopinath, D.; Husain, S. Antidiabetic and Oxidative Stress Ameliorative Potential of Ethanolic Extract of Pedicularis longiflora Rudolph. Int. J. Pharmacol. 2016, 12, 177-187. [CrossRef]

8. Li, M.X.; He, X.R.; Tao, R.; Cao, X. Phytochemistry and Pharmacology of the Genus Pedicularis Used in Traditional Chinese Medicine. Am. J. Chin. Med. 2014, 42, 1071-1098. [CrossRef] [PubMed]

9. USDA (2019) Natural Resources Conservation Service Report. Available online: www.plants.usda.gov/java/ ClassificationServlet? source=display\&classid=PEDIC (accessed on 15 May 2019).

10. Üçüncü, O.; Baltaci, C.; Ilter, S.M. Chemical composition, antimicrobial and antioxidant aactivities of essential oil from Pedicularis condensata BIEB. Hittite J. Sci. Engineer. 2016, 3, 105-109. [CrossRef]

11. Khodaie, L.; Bamdad, S.; Delazar, A.; Nazemiyeh, H. Antioxidant, Total Phenol and Flavonoid Contents of Two Pedicularis L. Species from Eastern Azerbaijan, Iran. BioImpacts 2012, 2, 43-57.

12. Khodaei, L.; Delazar, A.; Nazemiyeh, H.; Asnaashari, S.; Nahar, L.; Sarker, S.D. Composition of the Volatile Oils of the Aerial Parts of Pedicularis sibthorpii and P. wilhelmsiana Growing in Iran. J. Essent. Oil Bear. Plants 2012, 15, 352-356. [CrossRef]

13. Morteza-Semnani, K.; Saeedi, M.; Akbarzadeh, M. Chemical Composition of the Essential Oil of Pedicularis sibthorpii Boiss. J. Essent. Oil Bear. Plants 2014, 17, 1303-1307. [CrossRef]

14. Frezza, C.; Venditti, A.; Serafini, M.; Bianco, A. Phytochemistry, Chemotaxonomy, Ethnopharmacology, and Nutraceutics of Lamiaceae. Stud. Nat. Prod. Chem. 2019, 62, 125-178.

15. Akdemir, Z.S.; Çalıs, I.; Doga, T.R. Iridoid and phenylpropanoid glycosides from Pedicularis comosa var. acmodonta Boiss. J. Pharma. 1992, 2, 63-70.

16. Gao, J.J.; Jia, Z.J.; Liu, Z.M. Studies on the monoterpene iridoid glycosides from Pedicularis alaschanica. Chin. Chem. Lett. 1993, 14, 366-377.

17. Gao, J.; Jia, Z.J. Lignan, iridoid and phenylpropanoid glycosides from Pedicularis alaschanica Maxim (Scrophulariaceae). Indian J. Chem. B 1995, 34, 466-468.

18. Yuan, C.S.; Sun, X.B.; Zhao, P.H.; Cao, M.A. Antibacterial constituents from Pedicularis armata. J. Asian Nat. Prod. Res. 2007, 9, 673-677. [CrossRef]

19. Su, B.N.; Zhai, J.J.; Jia, Z.J. New Iridoids from Pedicularis artselaeri. J. Asian Nat. Prod. Res. 1998, 1, $103-109$. [CrossRef]

20. Su, B.; Ma, L.; Jia, Z. Iridoid and Phenylpropanoid Glycosides from Pedicularis artselaeri. Planta Medica 1998, 64, 720-723. [CrossRef]

21. Yatoo, M.I.; Dimri, U.; Gopalakrishnan, A.; Karthik, K.; Gopi, M.; Khandia, R.; Saminathan, M.; Saxena, A.; Alagawany, M.; Farag, M.R.; et al. Beneficial health applications and medicinal values of Pedicularis plants: A review. Biomed. Pharmacother. 2017, 95, 1301-1313. [CrossRef]

22. Schneider, M.J.; Lynch, J.D.; Deutsch, L.; Duda, C.M.; Green, J.C.; Mcpeak, D. Iridoid glycosides of Pedicularis. Biochem. Syst. Ecol. 1996, 24, 793-794. [CrossRef] 
23. Stermitz, F.R.; Suess, T.R.; Fink, N.H.; Puzziferri, N. Phytochemical Screening of Some Rocky Mountain Plants. J. Nat. Prod. 1981, 44, 693-695. [CrossRef]

24. Jung, H.J.G.; Batzli, G.O.; Seigler, D.S. Patterns in the phytochemistry of arctic plants. Biochem. Syst. Ecol. 1979, 7, 203-209. [CrossRef]

25. Yang, J.Q.; He, W.J.; Tan, N.H.; Chu, H.B.; Zhang, Y.M.; Mei, W.L.; Dai, H.F. Chemical constituents of Pedicularis cephalantha Franch and P. siphonantha Don. Nat. Prod. Res. Dev. 2009, 21, 600-603.

26. Murai, Y.; Tsukasa, I. Phenolic compounds in the leaves of Pedicularis chamissonis in Japan Bull. Natl. Mus. Nat. Sci. Ser. B 2015, 41, 131-136.

27. Yang, L.; Wang, C.Z.; Jia, Z.J. Iridoids in roots of Pedicularis chinensis. Phytochemistry 1995, 40, 491-494.

28. Wang, C.Z.; Jia, Z.J. Iridoid, lignan and phenylpropanoid glycosides from Pedicularis chinensis. J. Lanzhou Univers. (Nat. Sci.) 1996, 32, 64-68.

29. Akdemir, Z.; Çali, H.; Junior, P. Iridoid and phenylpropanoid glycosides from Pedicularis condensata. Phytochemistry 1991, 30, 2401-2402.

30. Schneider, M.J.; Stermitz, F.R. Uptake of host plant alkaloids by root parasitic Pedicularis species. Phytochemistry 1990, 29, 1811-1814. [CrossRef]

31. An, B.L.; Liu, Q.G.; Geng, Z.; Cheng, Z.G. Amino acids and trace elements in Pedicularis decora franch. J. Shanxi. Norm. Univ. (Nat. Sci.) 1995, 23, 62-64.

32. Guan, F.; Wang, X.J.; Yang, Y. Chemical studies on the Pedicularis decora Franch. J. Tradition. Chin. Med. 2004, 27, 920-921.

33. Li, C.; Zhang, C.Z.; Liu, M. Phenol glycosides from Pedicularis dicora. Chin. Tradition. Herb. Drug 1998, $29,11-12$.

34. Li, C.; Gu, D.S.; Zhang, C.Z.; Tao, B.Q. Iridoid glycosides from Pedicularis dicora Franch. China J. Chin. Mater. Med. 1999, 24, 40-41.

35. Li, C.; Zhang, C.Z. Nor-monoterpene glucides from Pedicularis dicora. Chin. Tradition. Herb. Drug 1999, 30, 482-484.

36. Zhang, C.Z.; Li, C.; Fen, S.L. Chemical studies on Pedicularis dicora. J. Lanzhou Med. Coll. 1991, 17, $199-200$.

37. Luo, D.Q.; Chu, H.B.; Tan, N.H.; Tian, X. Densispicnins A and B, Two Unusual Monoterpenes from Pedicularis densispica Franch. Heterocycles 2008, 75, 177. [CrossRef]

38. Chu, H.; He, W.; Zhang, Y.; Ji, C.; Tan, N. Flavonoids and nor-sesquiterpenes of Pedicularis densispica. China J. Chin. Mater. Medica 2011, 36, 2672-2675.

39. Chu, H.B.; Zeng, G.Z.; Zhu, M.J.; He, W.J.; Zhang, Y.M.; Tan, N.H. Chemical Constituents of Pedicularis densispica Franch. Z. Naturforsch. B 2011, 66, 641-646. [CrossRef]

40. Chu, H.B.; Tan, N.H. Iridoid Glycosides from Pedicularis dolichocymba Hand.-Mazz. J. Integr. Plant. Biol. 2006, 48, 1250-1253. [CrossRef]

41. Chu, H.B.; Tan, N.H.; Xiong, J.; Zhang, Y.M.; Ji, C.J. Chemical constituents of Pedicularis dolichocymba Hand.-Mazz. Nat. Prod. Res. Dev. 2007, 19, 584-587.

42. Ubeav, K.; Yuldashev, P.K.; Yunusov, S.Y. Pedicularis olgae alkaloids. Uzbeksk. Khim. Zh. 1963, 7, 33-36.

43. Karanjit, N.; Shakya, K.; Pradhan, N.B.; Gautam, L.; Shakya, D. Acompilation report of preliminary phytochemical and biological screening of some medicinal plants of Nepal. Bull. Dept. Pl. Res. 2007, 29, 79-96.

44. Wangchuk, P.; Keller, P.A.; Pyne, S.G.; Taweechotipatr, M.; Tonsomboon, A.; Rattanajak, R.; Kamchonwongpaisan, S. Evaluation of an ethnopharmacologically selected Bhutanese medicinal plants for their major classes of Phytochemicals and biological activities. J. Ethnopharmacol. 2011, 137, 730-742. [CrossRef]

45. Yin, J.G.; Yuan, C.S.; Jia, Z.J. A new iridoid and other chemical constituents from Pedicularis kansuensis forma albiflora Li. Arch. Pharmacal Res. 2007, 30, 431-435. [CrossRef]

46. Yuan, C.S.; Zhang, Q.; Xie, W.D.; Yang, X.P.; Jia, Z.J. Iridoids from Pedicularis kansuensis forma albiflora. Pharmazie 2003, 34, 428-430. [CrossRef]

47. Jiang, T.F.; Qing, Y.O.; Yan, P.S. Separation and determination of phenyl-propanoid glycosides from Pedicularis species by capillary electrophoresis. J. Chromatogr. A 2003, 986, 163-167. [CrossRef]

48. Guarnaccia, R.; Madyastha, K.M.; Tegtmeyer, E.; Coscia, C.J. Geniposidic acid, an iridoid glucoside from Genipa americana. Tetrahedron Lett. 1972, 13, 5125-5127. [CrossRef]

49. Zhang, Z.X.; Xie, W.D.; Jia, Z.J. Glycosides from two Pedicularis species. Biochem. Syst. Ecol. 2008, 36, 467-472. [CrossRef] 
50. Zhang, B.B.; Shi, K.; Liao, Z.X.; Dai, Y.; Zou, Z.H. Phenylpropanoid glycosides and triterpenoid of Pedicularis kansuensis Maxim. Fitoterapia 2011, 82, 854-860. [CrossRef]

51. Venditti, A.; Frezza, C.; Serafini, M.; Bianco, A. Iridoids and phenylethanoid from Pedicularis kerneri Dalla Torre growing in Dolomites, Italy. Nat. Prod. Res. 2016, 30, 327-331. [CrossRef]

52. Berg, T.; Damtoft, S.; Jensen, S.R.; Nielsen, B.J.; Rickelt, L.F. Iridoid glucosides from Pedicularis. Phytochemistry 1985, 24, 491-493. [CrossRef]

53. Jia, Z.J.; Liu, Z.M.; Wang, C.Z. Phenylpropanoid and iridoid glycosides from Pedicularis lasiophrys. Phytochemistry 1992, 31, 263-266.

54. Wang, C.Z.; Jia, Z.J. Neolignan glycosides from Pedicularis longiflora. Planta Medica 1997, 63, $241-244$. [CrossRef]

55. Jia, Z.J.; Liu, Z.M. Phenylpropanoid and iridoid glycosides from Pedicularis longiflora. Phytochemistry 1992, 31, 3125-3127.

56. Zhang, L.; Yue, H.L.; Zhao, X.H.; Li, J.; Shao, Y. Separation of Four Phenylpropanoid Glycosides from a Chinese Herb by HSCCC. J. Chromatogr. Sci. 2014, 53, 860-865. [CrossRef]

57. Fujii, M.; Miyaichi, Y.; Tomiinori, T. Flavonoid, phenylethanoid and iridoid constituents of the whole plant of Pedicularis longiflora var. tubiformis. Planta Medica 1991, 61, 584. [CrossRef]

58. Ma, Z.K.; Niu, B.J.; Zhang, B.B.; Liao, Z.X. Chemical constituents from Pedicularis longiflora var. tubiformis. Chin. Tradition. Herb. Drug 2013, 44, 403-407.

59. Zhang, L.; Shao, Y.; Zhao, X.H.; Yue, H.L.; Chen, C.; Tao, Y.D.; Mei, L.J.; Zhou, G.Y. Chemical constituents of Pedicularis longiflora Rudolph. var. tubiformis (Klotz). Tsoong. Nat. Prod. Res. Dev. 2013, 25, 40-43.

60. Kang, J.G.; Jia, Z.J. The chemical constituents of Pedicularis muscicola Maxim. J. Lanzhou Univ. (Nat. Sci.) 1997, 33, 69-74.

61. Kang, J.; Jia, Z. Chemical constituents of Pedicularis muscicola Maxim. China J. Chin. Mater. Medica 1997, 22, 167-168.

62. Yang, L.C.; Chen, L.D. Studies on the chemical constituents of Pedicularis muscicola Maxim. China J. Chin. Mater. Med. 1992, 17, 485-487.

63. Akdemir, Z.; Caliş, I.; Junior, P. Iridoids and phenyipropanoid glycosides from Pedicularis nordmanniana. Planta Medica 1991, 57, 584-585. [CrossRef]

64. Sadikov, Y.J. Biologically Active Substances of Wild Medicinal Plants of Tajikistan; Academy of Sciences of the Republic of Tajikistan: Dushanbe, Tajikistan, 2003; p. 320.

65. Jia, Z.J.; Gao, J.J.; Liu, Z.M. Iridoid and phenylpropanoid glycosides from Pedicularis plicata Maxim. Indian J. Chem. B 1994, 33, 460-464.

66. Schneider, M.J.; Green, J.C.; McPeak, D. Proceroside, an iridoid glucoside from Pedicularis procera. Phytochemistry 1997, 46, 1097-1098. [CrossRef]

67. Schneider, M.J.; Romero, K.A. Iridoid glycosides from Pedicularis punctata. Abstr. Pap. Am. Chem. Soc. 1995, 210, 355.

68. Bazzaz, B.S.F.; Haririzadeh, G.; Imami, A.S.; Rashed, M.H. Survey of Iranian Plants for Alkaloids, Flavonoids, Saponins, and Tannins (Khorasan Province). Int. J. Pharmacogn. 1997, 35, 17-30. [CrossRef]

69. Chu, H.B.; Tan, N.H.; Zhang, Y.M. Chemical constituents from Pedicularis rex C. B. Clarke. Z. Naturforsch. B 2007, 62, 1465-1470. [CrossRef]

70. Venditti, A.; Frezza, C.; Sciubba, F.; Foddai, S.; Serafini, M.; Nicoletti, M.; Bianco, A. Secoiridoids and other chemotaxonomically relevant compounds in Pedicularis: Phytochemical analysis and comparison of Pedicularis rostratocapitata Crantz and Pedicularis verticillata L. from Dolomites. Nat. Prod. Res. 2016, 30, 1698-1705. [CrossRef]

71. Sharopov, F.; Setzer, W.N. Medicinal Plants of Tajikistan; Egamberdieva, D., Öztürk, M., Eds.; Vegetation of Central Asia and Environs; Springer: Berlin, Germany, 2019.

72. Stermitz, F.R.; Belofsky, G.N.; Ng, D.; Singer, M.C. Quinolizidine alkaloids obtained by Pedicularis semibarbata (Scrophulariaceae), from Lupinus fulcratus (Leguminosae) fail to influence the specialist herbivore Euphydrya seditha (Lepidotera). J. Chem. Ecol. 1989, 15, 2521-2530. [CrossRef]

73. Wang, C.Z.; Jia, Z.J.; Shen, X.M. Phenylpropanoid, neolignan and iridoid glycosides from Pedicularis semitorta. Indian J. Chem. 1997, 36, 150-153.

74. Khodaie, L.; Delazar, A.; Lotfipour, F.; Nazemiyeh, H.; Asnaashari, S.; Moghadam, S.B.; Nahar, L.; Sarker, S.D. Phytochemistry and bioactivity of Pedicularis sibthorpii growing in Iran. Rev. Bras. Farm. 2012, 22, 1268-1275. [CrossRef] 
75. Jia, Z.J.; Liu, Z.; Wang, C. Phenypropanoid and iridoid glycosides from P. spicata. Phytochemistry 1991, 30, 3745-3747.

76. Zheng, R.L.; Wang, P.F.; Li, J.; Liu, Z.M.; Jia, Z.J. Inhibition of the autoxidation of linoleic acid by phenylpropanoid glycosides from Pedicularis in micelles. Chem. Phys. Lipids 1993, 65, 151-154. [CrossRef]

77. Liu, Z.M.; Jia, Z.J. Phenylpropanoid and iridoid glycosides from Pedicularis striata. Phytochemistry 1991, 30, 1341-1344.

78. Liu, Z.M.; Wang, C.Z.; Jia, Z.J. Chemical constituents from Pedicularis striata. J. Lanzhou Univ. (Nat. Sci.) 1994, 30, 62-65.

79. Mu, P.; Gao, X.; Jia, Z.J.; Zheng, R.L. Natural antioxidant pedicularioside G inhibits angiogenesis and tumourigenesis in vitro and in vivo. Basic Clin. Pharmacol. Toxicol. 2008, 102, 30-34. [CrossRef]

80. Gao, J.J.; Jia, Z.J.; Codina, C. An eremophilane sesquiterpenoid from Pedicularis striata Pall ssp. arachnoidea. Phytochemistry 1996, 43, 1411-1412. [CrossRef]

81. Gao, J.; Yang, L.; Jia, Z. A New Eremophilane Sesquiterpenoid and a New Iridoid from Pedicularis striata subsp. arachnoides. Planta Medica 1997, 63, 248-250. [CrossRef]

82. Jia, Z.J.; Gao, J.J. Phenylpropanoid glycosides from Pedicularis striata pall ssp. arachnoidea. Phytochemistry 1993, 34, 1188-1190.

83. Wang, C.Z.; Jia, Z.J. Neolignan glucosides from Pedicularis torta. Chin. Chem. Lett. 1996, 7, 141-144.

84. Wang, C.Z.; Jia, Z.J. Lignan, phenylpropanoid and iridoid glycosides from Pedicularis torta. Phytochemistry 1997, 45, 159-166. [CrossRef]

85. Yang, L.R.; Xiong, J.; Tan, N.H.; Chu, H.B.; Xu, L.; Li, M.Y. Chemical constituents of Pedicularis tricolor (Scrophulariaceae). Acta Bot. Yunnan 2006, 28, 553-557.

86. Liu, L.F.; Yao, M.J.; Li, M.Y.; Wu, X.Z.; Yuan, C.S. Iridoid Derivatives with Cytotoxic Activity from Pedicularis uliginosa Bunge. Chem. Biodivers. 2019, 16, e1800524. [CrossRef]

87. Shao, M.H.; Dai, W.; Yuan, S.W.; Lu, Y.; Chen, D.F.; Wang, Q.; Shao, M.; Yuan, S.; Chen, D. Iridoids from Pedicularis verticillata and Their Anti-Complementary Activity. Chem. Biodivers. 2018, 15, e1800033. [CrossRef]

88. Su, B.N.; Yang, L.; Jia, Z.J. Neolignan, phenylpropanoid and iridoid glycosides from Pedicularis verticillata. Phytochemistry 1997, 45, 1271-1273. [CrossRef]

89. Venditti, A. What is and what should never be: Artifacts, improbable phytochemicals, contaminants and natural products. Nat. Prod. Res. 2018, 1-18. [CrossRef] [PubMed]

90. Venditti, A. Artifacts in natural products studies. An old and underestimated re-emerging problem. Nat. Prod. Res. 2018, 32, I-II. [CrossRef] [PubMed]

91. McGarbey, D.; Croteau, R. Terpenoid Metabolism. Plant Cell 1995, 7, 1015-1026.

92. Damtoft, S.; Jensen, S.R.; Nielsen, B.J. Biosynthesis of iridoid glucosides in Lamium album. Phytochemistry 1992, 31, 135-137. [CrossRef]

93. Cheminat, A.; Zawatzky, R.; Becker, H.; Brouillard, R. Caffeoyl conjugates from Echinacea species: Structures and biological activity. Phytochemistry 1998, 27, 2787-2794. [CrossRef]

94. Machida, K.; Nakano, Y.; Kikuchi, M. Phenolic glycosides from Viburnum dilatatum. Phytochemistry 1991, 30, 2013-2014. [CrossRef]

95. Kitagawa, S.; Hisada, S.; Nishibe, S. Phenolic compounds from Forsythia leaves. Phytochemistry 1984, 23, 1635-1636. [CrossRef]

96. Ravn, H.; Nishibe, S.; Sasahara, M.; Xuebo, L. Phenolic compounds from Plantago asiatica. Phytochemistry 1990, 29, 3627-3631. [CrossRef]

97. Gardner, D.R.; Narum, J.; Zook, D.; Stermitz, F.R. New Iridoid Glucosides from Castilleja and Besseya: 6-Hydroxyadoxoside and 6-Isovanillylcatapol. J. Nat. Prod. 1987, 50, 485-489. [CrossRef]

98. Lin, Y.L.; Kuo, Y.H. A New Glycoside, Brachynoside, Isolated from Clerodendron brachyanthum SCHAUER. Chem. Pharm. Bull. 1992, 40, 1928-1929. [CrossRef]

99. Jensen, S.R. Systematic Implications of the Distribution of Iridoids and Other Chemical Compounds in the Loganiaceae and Other Families of the Asteridae. Ann. Mo. Bot. Gard. 1992, 79, 284. [CrossRef]

100. Frezza, C.; Venditti, A.; Di Cecco, M.; Ciaschetti, G.; Serafini, M.; Bianco, A. Iridoids and phenylethanoid glycosides from the aerial parts of Ajuga tenorei, an endemic Italian species. Nat. Prod. Res. 2017, 31, 218-223. [CrossRef] [PubMed] 
101. Frezza, C.; Venditti, A.; Matrone, G.; Serafini, I.; Foddai, S.; Bianco, A.; Serafini, M. Iridoid glycosides and polyphenolic compounds from Teucrium chamaedrys L. Nat. Prod. Res. 2018, 32, 1583-1589. [CrossRef] [PubMed]

102. Venditti, A.; Serrilli, A.M.; Bianco, A. Iridoids from Bellardia trixago (L.) All. Nat. Prod. Res. 2013, 27, $1413-1416$. [CrossRef] [PubMed]

103. Venditti, A.; Bianco, A.; Nicoletti, M.; Quassinti, L.; Bramucci, M.; Lupidi, G.; Vitali, L.A.; Petrelli, D.; Papa, F.; Vittori, S.; et al. Phytochemical analysis, biological evaluation and micromorphological study of Stachys alopecuros (L.) Benth. subsp. divulsa (Ten.) Grande endemic to central Apennines, Italy. Fitoterapia 2013, 90, 94-103. [CrossRef] [PubMed]

104. Frezza, C.; Celona, D.; Bianco, A.; Serafini, M.; Fiorini, D.; Maggi, F.; Celenza, G.; Ferraro, S.; Venditti, A.; Cianfaglione, K.; et al. Polar constituents, protection against reactive oxygen species, and nutritional value of Chinese artichoke (Stachys affinis Bunge). Food Chem. 2017, 221, 473-481.

105. Venditti, A.; Frezza, C.; Bianco, A.; Serafini, M.; Cianfaglione, K.; Nagy, D.U.; Iannarelli, R.; Caprioli, G.; Maggi, F. Polar Constituents, Essential Oil and Antioxidant Activity of Marsh Woundwort (Stachys palustris L.). Chem. Biodivers. 2017, 14, e1600401. [CrossRef]

106. Jiménez, C.; Riguera, R. Phenylethanoid glycosides in plants: Structure and biological activity. Nat. Prod. Rep. 1994, 11, 591-606. [CrossRef]

107. Xu, W.H.; Zhao, P.; Wang, M.; Liang, Q. Naturally occurring furofuran lignans: Structural diversity and biological activities. Nat. Prod. Res. 2017. [CrossRef]

108. Frezza, C.; Venditti, A.; Sciubba, F.; Tomai, P.; Antonetti, M.; Franceschin, M.; Di Cocco, M.E.; Gentili, A.; Delfini, M.; Serafini, M.; et al. Phytochemical profile of Euphorbia peplus L. collected in Central Italy and NMR semi-quantitative analysis of the diterpenoid fraction. J. Pharm. Biomed. Anal. 2018, 160, 152-159. [CrossRef]

109. Iwashina, T. The Structure and Distribution of the Flavonoids in Plants. J. Plant. Res. 2000, 113, $287-299$. [CrossRef]

110. Bohm, B.A.; Stuessy, T.F. Flavonoids of the Sunflower Family (Asteraceae); Springer Science and Business Media LLC: Berlin, Germany, 2001.

111. Caprioli, G.; Alunno, A.; Beghelli, D.; Bianco, A.; Bramucci, M.; Frezza, C.; Iannarelli, R.; Papa, F.; Quassinti, L.; Sagratini, G.; et al. Polar Constituents and Biological Activity of the Berry-Like Fruits from Hypericum androsaemum L. Front. Plant. Sci. 2016, 7, 62. [CrossRef]

112. Mesquita, A.A.; Corréa, D.D.B.; De Pádua, A.P.; Guedes, M.L.; Gottlieb, O.R. Flavonoids from four compositae species. Phytochemistry 1986, 25, 1255-1256. [CrossRef]

113. Venditti, A.; Frezza, C.; Majd Zadeh, S.M.; Foddai, S.; Serafini, M.; Bianco, A. Secondary metabolites from Teucrium polium L. collected in Southern Iran. Arab. J. Med. Arom. Pl. 2017, 3, 108-123.

114. Venditti, A.; Bianco, A. Secondary metabolites of Hypericum richer Vill. collected in Central Italy: Chemotaxonomy and ethnomedicinal relevance. Tr. Phytochem. Res. 2018, 2, 155-162.

115. Venditti, A.; Ballero, M.; Serafini, M.; Bianco, A. Polar compounds from Parentucellia viscosa (L.) Caruel from Sardinia. Nat. Prod. Res. 2015, 29, 602-606. [CrossRef]

116. Rasmussen, L.S.; Rank, C.; Jensen, S.R. Transfer of iridoid glucosides from host plant Galium verum to hemiparasitic Euphrasia stricta. Biochem. Syst. Ecol. 2006, 34, 763-765. [CrossRef]

117. D'Ambrosio, M.; Ciocarlan, A.; Aricu, A. Minor acetylated metabolites from Euphrasia rostkoviana. Nat. Prod. Res. 2018, 1-6. [CrossRef]

118. Venditti, A.; Frezza, C.; Foddai, S.; Serafini, M.; Nicoletti, M.; Bianco, A. Chemical Traits of Hemiparasitism in Odontites luteus. Chem. Biodivers. 2017, 14, e1600416. [CrossRef]

119. Jiangsu New Medical College. Chinese Medicine Dictionary; Shanghai People's Publishing House: Shanghai, China, 1977; p. 286.

120. Manandhar, N.P. Ethnobotanical note on folk-lore remedies of Baglung district, Nepal. Contrib.Nepal Stud. J. 1993, 20, 183-196.

121. Angmo, K.; Adhikari, B.S.; Rawat, G.S. Changing aspects of Traditional Healthcare System in Western Ladakh, India. J. Ethnopharmacol. 2012, 143, 621-630. [CrossRef]

122. Balami, N.P. Ethnomedicinal uses of plants among the Newar community of Pharping village of Kathmandu district, Nepal. Tribhuvan Univers. J. 2004, 24, 1-10. [CrossRef]

123. Duffy Davis, J.; Banack, S.A. Ethnobotany of the Kiluhikturmiut Inuinnait of Kugluktuk, Nunavut, Canada. Ethnobiol. Lett. 2012, 3, 78-90. [CrossRef] 
124. Ballabh, B.; Chaurasia, O.P. Medicinal plants of cold desert Ladakh used in the treatment of stomach disorders. Indian J. Tradition. Know. 2009, 8, 185-190.

125. Ballabh, B.; Chaurasia, O.P. Herbal Formulations from Cold Desert Plants Used For Gynecological Disorders. Ethnobot. Res. Appl. 2011, 9, 59-66. [CrossRef]

126. Çakır, E.A. Traditional knowledge of wild edible plants of Iğdır Province (East Anatolia, Turkey). Acta Soc. Bot. Pol. 2017, 86, 1-20.

127. Wang, P.; Kang, J.; Zheng, R.; Yang, Z.; Lu, J.; Gao, J.; Jia, Z. Scavenging effects of phenylpropanoid glycosides from Pedicularis on superoxide anion and hydroxyl radical by the Spin trapping method. Biochem. Pharmacol. 1996, 51, 687-691. [CrossRef]

128. Wangchuk, P.; Dorji, S.; Ugyen; Thinley, J.; Afaq, S.H. High altitude plants used in Bhutanese traditional medicine (gSo-ba-rig-pa). Ethnobot. 2008, 20, 54-64.

129. Wali, R.; Rahman, K.; Raja, N.I.; Qureshi, R.; Mashwani, Z.U.R. A quantitative medico-botanical expedition of Fairy Meadows National Park, Diamir, Gilgit Baltistan, Pakistan. bioRxiv 2018, 507848.

130. Malla, B.; Gauchan, D.P.; Chhetri, R.B. An ethnobotanical study of medicinal plants used by ethnic people in Parbat district of western Nepal. J. Ethnopharmacol. 2015, 165, 103-117. [CrossRef]

131. Pande, P.C.; Tiwari, L.; Pande, H.C. Ethnoveterinary plants of Uttaranchal-A review. Indian J. Tradition. Know. 2007, 6, 444-458.

132. Singh, G.; Rawat, G.S. Ethnomedicinal survey of Kedarnath wildlife sanctuary in Western Himalaya, India. Indian J. Fund Appl. Life Sci. 2011, 1, 35-46.

133. Uprety, Y.; Lacasse, A.; Asselin, H. Traditional uses of medicinal plants from the Canadian Boreal Forest for the management of chronic pain syndromes. Pain Pract. 2016, 16, 459-466. [CrossRef]

134. Mentsikhang, L.K. Himalayan Doctors and Healing Herbs: The Amchi Tradition and Medicinal Plants of Mustang; Mera Publications: Kathmandu, Nepal, 2005.

135. Wangchuk, P. Bioactive alkaloids from medicinal plants of Bhutan. Ph.D. Thesis, Department of Chemistry, University of Wollongong, Wollongong, Australia, 2004.

136. Rinchen, T.; Pant, S. Ethnopharmacological uses of plants among inhabitants surrounding Suru and Zanskar valleys of cold desert, Ladakh. Int. J. Pharma. Bio Sci. 2014, 5, 486-494.

137. Shang, X.; Tao, C.; Miao, X.; Wang, D.; Tangmuke; Dawa; Wang, Y.; Yang, Y.; Pan, H. Ethno-veterinary survey of medicinal plants in Ruoergai region, Sichuan province, China. J. Ethnopharmacol. 2012, 142, 390-400. [CrossRef]

138. O'Neill, A.R.; Rana, S.K. An ethnobotanical analysis of parasitic plants (Parijibi) in the Nepal Himalaya. J. Ethnobiol. Ethnomed. 2016, 12, 79. [CrossRef]

139. Kapahi, B.; Srivastava, T.; Sarin, Y. Traditional medicinal plants of gurez (kashmir)—An ethnobotanical study. Anc. Sci. Life 1993, 13, 119-124.

140. Singh, K.; Lal, B. Ethnomedicines used against four common ailments by the tribal communities of Lahaul-Spiti in western Himalaya. J. Ethnopharmacol. 2008, 115, 147-159. [CrossRef]

141. Devi, U.; Seth, M.K.; Sharma, P.; Rana, J.C. Study on ethnomedicinal plants of kibber wildlife sanctuary: A cold desert in Trans Himalaya, India. J. Med. Pl. Res. 2013, 7, 3400-3419.

142. Dutt, H.C.; Bhagat, N.; Pandita, S. Oral traditional knowledge on medicinal plants in jeopardy among Gaddi shepherds in hills of northwestern Himalaya, J\&K, India. J. Ethnopharmacol. 2015, 168, 337-348.

143. Gairola, S.; Sharma, J.; Bedi, Y.S. A cross-cultural analysis of Jammu, Kashmir and Ladakh (India) medicinal plant use. J. Ethnopharmacol. 2014, 155, 925-986. [CrossRef]

144. Salim, M.A.; Ranjitkar, S.; Hart, R.; Khan, T.; Ali, S.; Kiran, C.; Parveen, A.; Batool, Z.; Bano, S.; Xu, J. Regional trade of medicinal plants has facilitated the retention of traditional knowledge: Case study in Gilgit-Baltistan Pakistan. J. Ethnobiol. Ethnomed. 2019, 15, 1-33. [CrossRef]

145. Singh, K.N. Traditional knowledge on ethnobotanical uses of plant biodiversity: A detailed study from the Indian western Himalaya. Biodivers. Res. Conserv. 2012, 28, 63-77. [CrossRef]

146. Bano, A.; Ahmad, M.; Zafar, M.; Sultana, S.; Rashid, S.; Khan, M.A. Ethnomedicinal knowledge of the most commonly used plants from Deosai Plateau, Western Himalayas, Gilgit Baltistan, Pakistan. J. Ethnopharmacol. 2014, 155, 1046-1052. [CrossRef]

147. Kayani, S.; Ahmad, M.; Sultana, S.; Shinwari, Z.K.; Zafar, M.; Yaseen, G.; Hussain, M.; Bibi, T. Ethnobotany of medicinal plants among the communities of Alpine and Sub-alpine regions of Pakistan. J. Ethnopharmacol. 2015, 164, 186-202. [CrossRef] 
148. Yoo, S.J.; Yim, D.S.; Lee, S.Y. Biological activities of Pedicularis resupinata var. oppositifolia. Korean J. Pharmacog. 1993, 24, 258-262.

149. Marles, R.; Farnsworth, N. Antidiabetic plants and their active constituents. Phytomedicine 1995, 2, 137-189. [CrossRef]

150. Toba, S. Plant names in Khaling: A study in ethnobotany and village economy. Kailash 1975, 3, 145-169.

151. Rokaya, M.B.; Münzbergová, Z.; Shrestha, M.R.; Timsina, B. Distribution patterns of medicinal plants along an elevational gradient in central Himalaya, Nepal. J. Mt. Sci. 2012, 9, 201-213. [CrossRef]

152. Wagner, A.; Kriechbaum, M.; Koch, M.A. Applied Vulnerability Assessment of Useful Plants: A case study of Tibetan Medicinal Plants from Nepal. Botanische Jahrbücher 2008, 127, 359-387. [CrossRef]

153. Magsar, U.; Nyamsuren, K.; Khadbaatar, S.; Tovuudorj, M.E.; Baasansuren, E.; Indree, T.; Lkhagvadorj, K.; Kwon, O. Survey of medicinal plants in the Khuvsgul and Khangai Mountain regions of Mongolia. J. Ecol. Environ. 2017, 41, 1-5. [CrossRef]

154. Qiu, S.; Sun, H.; Zhang, A.H.; Xu, H.Y.; Yan, G.L.; Han, Y.; Wang, X.J. Natural alkaloids: Basic aspects, biological roles, and future perspectives. Chin. J. Nat. Med. 2014, 12, 401-406. [CrossRef]

155. Hu, L.L. Protection of Pedicularis on liver lesion of mice induced by Alloxan. J. Ankang Univ. 2007, 19, 72-74.

156. Dulger, B.; Ugurlu, E. Evaluation of Antimicrobial Activity of Some Endemic Scrophulariaceae. Members from Turkey. Pharm. Biol. 2005, 43, 275-279. [CrossRef]

157. Yang, J.X.; Tian, J.W.; Li, F.R. Influence on antioxidative ability of Taibaishen in mice. Northwest Pharmaceut. J. 2001, 16, 209-211.

158. Gao, M.; Li, Y.; Yang, J. Protective effect of Pedicularis decora Franch root extracts on oxidative stress and hepatic injury in alloxan-induced diabetic mice. J. Med. Pl. Res. 2011, 5, 5848-5856.

159. Gonchig, E.; Erdenebat, S.; Togtoo, O.; Bataa, S.; Gendaram, O.; Kim, Y.S.; Ryu, S.Y. Antimicrobial activity of Mongolian medicinal plants. Nat. Prod. Sci. 2008, 14, 1-5.

160. Moreno-Escobar, J.A.; Bazaldúa, S.; Villarreal, M.L.; Bonilla-Barbosa, J.R.; Mendoza, S.; López, V.R. Cytotoxic and antioxidant activities of selected Lamiales species from Mexico. Pharm. Biol. 2011, 49, 1243-1248. [CrossRef]

161. Cushnie, T.T.; Cushnie, B.; Lamb, A.J. Alkaloids: An overview of their antibacterial, antibiotic-enhancing and antivirulence activities. Int. J. Antimicrob. Agents 2014, 44, 377-386. [CrossRef]

162. Moreira, R.; Pereira, D.M.; Valentão, P.; Andrade, P.B. Pyrrolizidine Alkaloids: Chemistry, Pharmacology, Toxicology and Food Safety. Int. J. Mol. Sci. 2018, 19, 1668. [CrossRef]

163. Korkina, L.; Kostyuk, V.; De Luca, C.; Pastore, S. Plant phenylpropanoids as emerging anti-inflammatory agents. Mini Rev. Med. Chem. 2011, 11, 823-835. [CrossRef]

164. Muller-Harvey, I.; McAllan, A.B. Tannins: Their biochemistry and nutritional properties. Adv. Plant Cell Biochem. Biotechnol. 1992, 1, 151-217.

165. Fu, G.; Pang, H.; Wong, Y.H. Naturally occurring phenylethanoid glycosides: Potential leads for new therapeutics. Curr. Med. Chem. 2008, 15, 2592-2613. [CrossRef]

166. Panche, A.N.; Diwan, A.D.; Chandra, S.R. Flavonoids: An overview. J. Nutr. Sci. 2016, 5, 1-15. [CrossRef]

167. Steiner, L.F.; Summerland, S.A. Xanthone as an Ovicide and Larvicide for the Codling moth. J. Econ. Èntomol. 1943, 36, 435-439. [CrossRef]

168. Tundis, R.; Loizzo, M.R.; Menichini, F.; Statti, G.; Menichini, F. Biological and Pharmacological Activities of Iridoids: Recent Developments. Mini Rev. Med. Chem. 2008, 8, 399-420. [CrossRef]

169. Ghisalberti, E. Biological and pharmacological activity of naturally occurring iridoids and secoiridoids. Phytomedicine 1998, 5, 147-163. [CrossRef]

170. Li, X.X.; Zhou, Z.K. Endemic Wild Ornamental Plants from Northwestern Yunnan, China. HortScience 2005, 40, 1612-1619. [CrossRef]

171. Clark, C. Inuit Ethnobotany and Ethnoecology in Nunavik and Nunatsiavut, Northeastern Canada. Ph.D. Thesis, Univeristy of Montreal, Montreal, QC, Canada, 2012.

172. Tardío, J.; Morales, R.; Pardo-De-Santayana, M.; Pardo-De-Santayana, M.; Pardo-De-Santayana, M. Ethnobotanical review of wild edible plants in Spain. Bot. J. Linn. Soc. 2006, 152, 27-71. [CrossRef]

173. Menges, E.S. Population Viability Analysis for an Endangered Plant. Conserv. Biol. 1990, 4, 52-62. [CrossRef]

174. Cho, W.B.; Choi, I.S.; Choi, B.H. Development of microsatellite markers for the endangered Pedicularisis hidoyana (Orobanchaceae) using next-generation sequencing source. Appl. Pl. Sci. 2003, 3, 1-3. 
175. Ali, H. Conservation Issues of High Altitude Medicinal Plants of Pakistan. In Proceedings and Abstract of the 2nd International Symposium on Kaz Mountains (Mount Ida) and Edremit; Edremit, Turkey, 3-5 May 2013, Ikes2013: Izmir, Turkey, 2013; pp. 82-93. Available online: https://www.academia.edu/4814159/Proceedings-The_Second_International_Symposium_on_Kaz_ Mountains_and_Edremit-Human_-_Environment_Interactions (accessed on 15 July 2019).

176. Liu, Y.Y.; Hu, Y.K.; Yu, J.M.; Li, K.H.; Gao, G.G.; Wang, X. Study on harmfulness of Pedicularis myriophylla and its control measures. Arid Zone Res. 2008, 25, 778-782.

177. Smith-Kuebel, C.; Lillybridge, T.R. Report on Sensitive plants and noxious weeds of the Wenatchee National Forest; The Cultural Resources and Vegetation Management Division of the Bonneville Power Administration: Portland, OR, USA, 1993.

178. Santisuk, T.; Chayamarit, K.; Pooma, R.; Suddee, S. Thailand Red Data: Plants; Office of Natural Resources and Environmental Policy and Planning: Bangkok, Thailand, 2006; pp. 1-256.

179. Conti, F.; Bartolucci, F. Specie a Rischio in Abruzzo. Elenco Delle Piante di Interesse Conservazionistico. In La Biodiversità vegetale in Abruzzo. Tutela e conservazione del patrimonio vegetale abruzzese; One Group Ed.: L'Aquila, Italy, 2012; pp. 81-109.

180. Kumar, A.; Mitra, M.; Adhikari, B.S.; Rawat, G.S. Flora of Niti Valley: A cold arid region of Nanda Devi Biosphere Reserve, Western Himalaya, India. Check List. 2016, 12, 1824. [CrossRef]

181. Yu, W.B.; Wang, H.; Li, D.Z. (2204) Proposal to conserve Pedicularis stenocorys against P. stenantha (Orobanchaceae). TAXON 2013, 62, 1066-1067. [CrossRef]

(C) 2019 by the authors. Licensee MDPI, Basel, Switzerland. This article is an open access article distributed under the terms and conditions of the Creative Commons Attribution (CC BY) license (http://creativecommons.org/licenses/by/4.0/). 